\title{
Pseudo-Helical and Helical Primary Structures of 1,2-Spiroannelated Four- and Five-Membered Rings: Syntheses and Chiroptical Properties
}

\author{
Tien Widjaja, ${ }^{1}$ Lutz Fitjer, ${ }^{1, *}$ Aritra Pal, ${ }^{2}$ Hans-Georg Schmidt, ${ }^{2}$ Mathias Noltemeyer, ${ }^{2}$ \\ Christian Diedrich, ${ }^{3}$ and Stefan Grimme ${ }^{3, *}$
}

\footnotetext{
${ }^{1}$ Institut für Organische und Biomolekulare Chemie, Universität Göttingen, Tammannstrasse 2, 37077 Göttingen, Germany, ${ }^{2}$ Institut für Anorganische Chemie, Universität Göttingen, Tammannstrasse 4, 37077 Göttingen, Germany, and ${ }^{3}$ Organisch Chemisches Institut (Abt. Theoretische Chemie), Universität Münster, Corrensstrasse 40, 48149 Münster, Germany
}

\section{Table of contents}

General

Experimental and spectral data of $\mathbf{2 0}$

Experimental and spectral data of $\mathbf{2 2}$

S4

Experimental and spectral data of $\mathbf{2 3}$

S5

Experimental of $\mathbf{1 6}$

S6

Experimental and spectral data of $\left(1 S, 5^{\prime} S, 11^{\prime} S\right)-(+)-\mathbf{2 6}$

S7

Experimental of (5R)-(-)-6

S7

Experimental of $(5 S)-(-)-7$ S8

Experimental of $\mathbf{2 9}$

Experimental of $\mathbf{3 0}$ S9

Experimental and spectral data of $\mathbf{3 1}$

Experimental of $\mathbf{3 2}$

Experimental and spectral data of $\left(4 R^{*}, 5 S^{*}\right)-34$

S10

Experimental and spectral data of $\mathbf{4 0}$

$\mathrm{S} 11$

$\begin{array}{ll}\text { Experimental and spectral data of }\left(4 S^{*}\right)-\mathbf{4 1} & \mathrm{S} 12\end{array}$

$\begin{array}{ll}\text { Experimental and spectral data of }\left(1 R^{*}, 4 S^{*}\right)-\mathbf{4 4} & \mathrm{S} 13\end{array}$

$\begin{array}{ll}\text { Experimental and spectral data for }\left(1 R^{*}, 4 S^{*}, 5 S^{*}\right)-\mathbf{4 5} & \mathrm{S} 14\end{array}$

$\begin{array}{ll}\text { Experimental and spectral data for }(1 S, 4 R)-\mathbf{5 1} \text { and }(1 S, 4 S)-\mathbf{5 3} & \mathrm{S} 14\end{array}$

$\begin{array}{ll}\text { Experimental of }(4 R)-(+)-\mathbf{4 1} \text { as prepared from }(1 S, 4 R)-\mathbf{5 1} & \mathrm{S} 15\end{array}$ 
Experimental of $(4 S)-(-)-\mathbf{4 1}$ as prepared from $(1 S, 4 S)-\mathbf{5 3} \quad S 16$

$\begin{array}{ll}\text { Experimental and spectral data of }(1 S, 4 R)-49 & \text { S17 }\end{array}$

$\begin{array}{ll}\text { Experimental of }(1 S, 4 R)-(-)-42 & \mathrm{~S} 17\end{array}$

$\begin{array}{ll}\text { Experimental of }(6 R, 7 R, 8 S)-(-)-43 & \mathrm{~S} 18\end{array}$

Experimental of $(6 R)-(-)-46 \quad S 18$

Experimental of $(P)-(-)-9 \quad S 18$

$\begin{array}{ll}\text { Experimental of }(1 R, 4 R)-48 & \text { S19 }\end{array}$

$\begin{array}{ll}\text { Experimental of }(5 R)-(-)-47 & \text { S19 }\end{array}$

$\begin{array}{ll}\text { Experimental of }(5 R)-(-)-8 & \text { S19 }\end{array}$

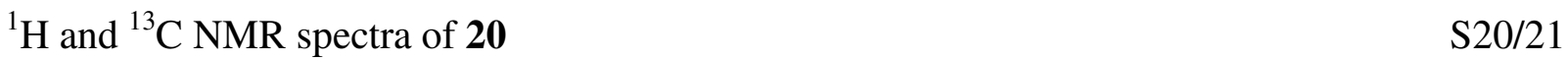

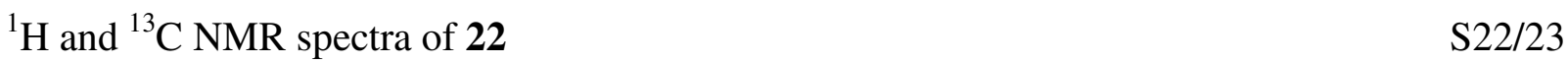

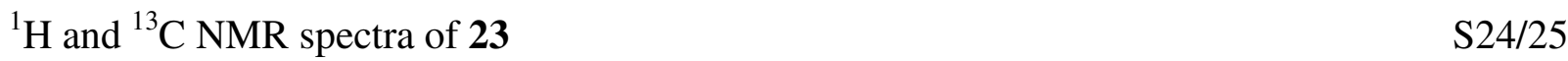

$\begin{array}{ll}{ }^{1} \mathrm{H} \text { and }{ }^{13} \mathrm{C} \text { NMR spectra of } \mathbf{1 5} & \text { S26/27 }\end{array}$

$\begin{array}{ll}{ }^{1} \mathrm{H} \text { and }{ }^{13} \mathrm{C} \text { NMR spectra of } 6 & \text { S28/29 }\end{array}$

${ }^{1} \mathrm{H}$ and ${ }^{13} \mathrm{C}$ NMR spectra of $\mathbf{2 4} \quad$ S30/31

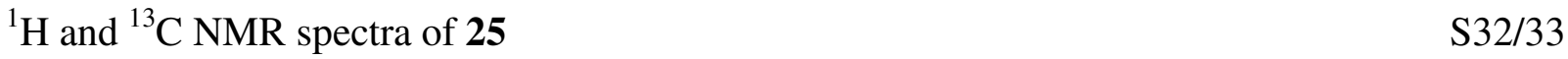

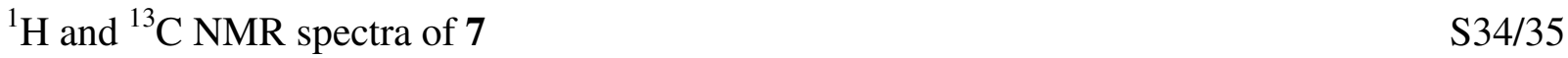

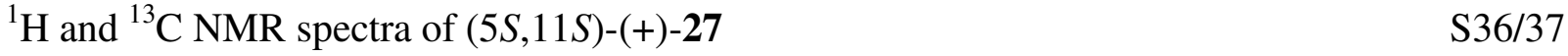

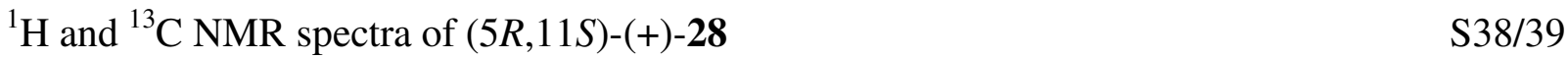

${ }^{1} \mathrm{H}$ and ${ }^{13} \mathrm{C}$ NMR spectra of $\left(1 S, 5^{\prime} S, 11^{\prime} S\right)-(+)-26 \quad S 40 / 41$

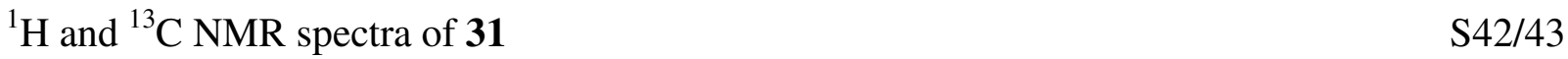

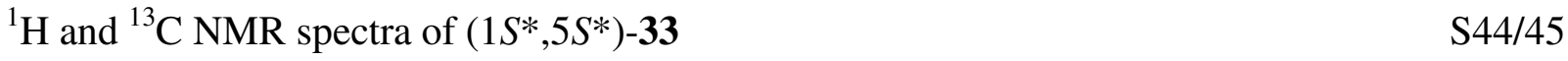

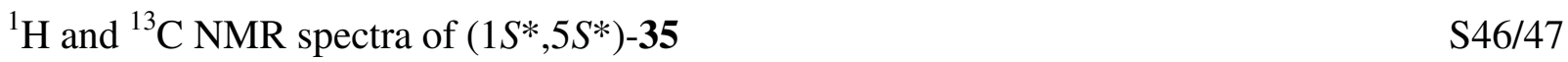

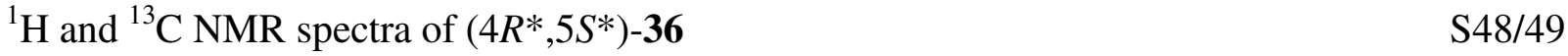

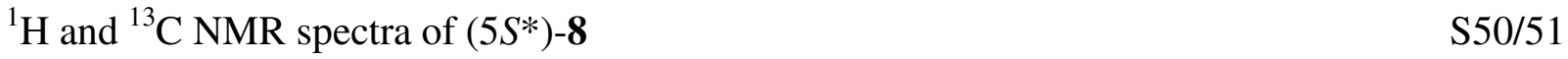

${ }^{1} \mathrm{H}$ and ${ }^{13} \mathrm{C}$ NMR spectra of $\left(4 R^{*}, 5 S^{*}\right)-34 \quad$ S52/53

${ }^{1} \mathrm{H}$ and ${ }^{13} \mathrm{C}$ NMR spectra of $\left(4 R^{*}, 5 S^{*}\right)-37 \quad \mathrm{~S} 54 / 55$

${ }^{1} \mathrm{H}$ and ${ }^{13} \mathrm{C}$ NMR spectra of $\left(3 S^{*}, 4 R^{*}, 5 S^{*}\right)-39 \quad \mathrm{~S} 56 / 57$

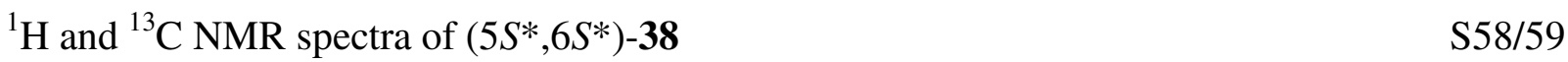

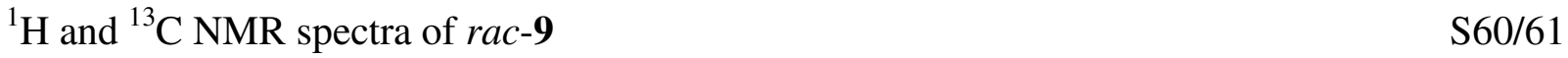

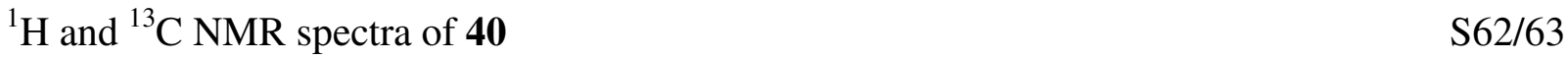

${ }^{1} \mathrm{H}$ and ${ }^{13} \mathrm{C}$ NMR spectra of (4S*)-41 $\quad$ S64/65

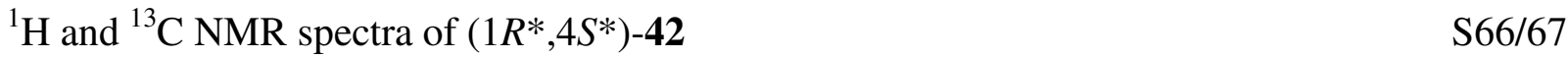

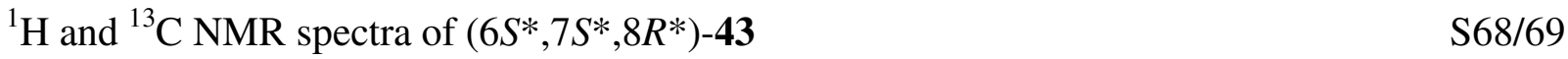

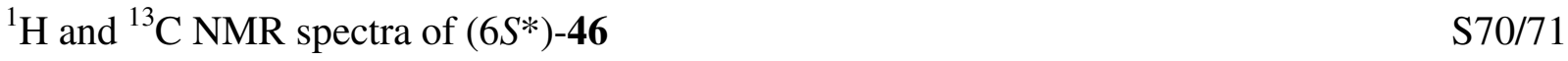

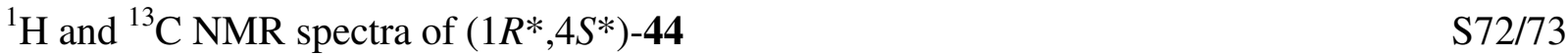


$\begin{array}{ll}{ }^{1} \mathrm{H} \text { and }{ }^{13} \mathrm{C} \text { NMR spectra of }\left(1 R^{*}, 4 S^{*}, 5 S^{*}\right)-45 & \mathrm{~S} 74 / 75\end{array}$

$\begin{array}{ll}{ }^{1} \mathrm{H} \text { and }{ }^{13} \mathrm{C} \text { NMR spectra of }\left(1 S^{*}, 4 S^{*}\right)-\mathbf{4 8} & \mathrm{S} 76 / 77\end{array}$

$\begin{array}{ll}{ }^{1} \mathrm{H} \text { and }{ }^{13} \mathrm{C} \text { NMR spectra of }\left(5 S^{*}\right)-47 & \text { S78/79 }\end{array}$

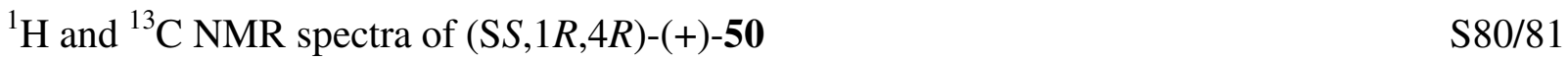

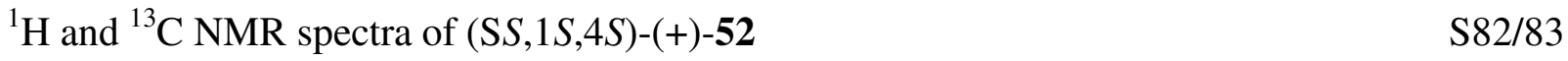

${ }^{1} \mathrm{H}$ and ${ }^{13} \mathrm{C}$ NMR spectra of $(1 S, 4 R)-(+)-51 \quad S 84 / 85$

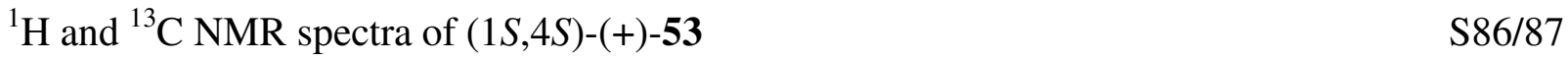

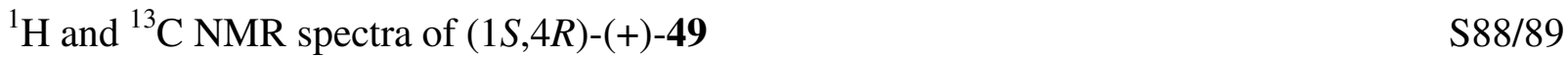

Crystallographic data of $\left(1 S, 5^{\prime} S, 11^{\prime} S\right)-(+)-\mathbf{2 6},\left(4 R^{*}, 5 S^{*}\right)-\mathbf{3 4},\left(6 S^{*}, 7 S^{*}, 8 R^{*}\right)-\mathbf{4 3} \quad \mathrm{S} 90$

Crystallographic data of $(1 S, 4 R)-(+)-\mathbf{4 9},(\mathrm{S} S, 1 R, 4 R)-\mathbf{5 0},(\mathrm{SS}, 1 S, 4 S)-\mathbf{5 2} \quad \mathrm{S} 91$

ORTEP plot of $\left(1 S, 5^{\prime} S, 11^{\prime} S\right)-(+)-26 \quad S 92$

ORTEP plot of $\left(4 R^{*}, 5 S^{*}\right)-\mathbf{3 4} \quad \mathrm{S} 93$

ORTEP plot of $\left(6 S^{*}, 7 S^{*}, 8 R^{*}\right)-\mathbf{4 3} \quad \mathrm{S} 94$

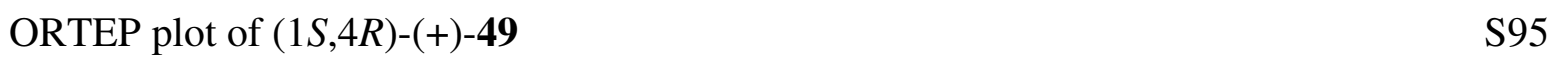

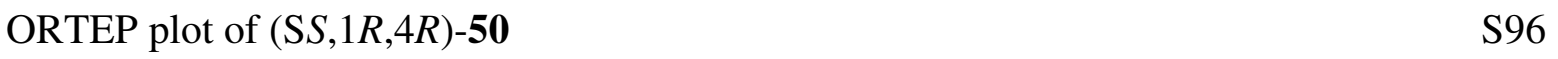

$\begin{array}{ll}\text { ORTEP plot of }(\mathrm{SS}, 1 S, 4 S)-\mathbf{5 2} & \mathrm{S} 97\end{array}$

Cartesian coordinates $(\AA)$ of the optimized structures (B97-D/TZVP) of $\quad$ S98/113

the $(P)$-helices of 1, 2, 3, 4-1 to 4-6, 5-1 to 5-6, 9-1 to 9-4, 10-1 and 10-2

General. IR-spectra were obtained with a standard instrument. ${ }^{1} \mathrm{H}$ and ${ }^{13} \mathrm{C}$ NMR spectra were obtained from spectrometers operating at 300,500 or $600 \mathrm{MHz}$ for ${ }^{1} \mathrm{H}$. As standards the following chemical shifts were used: $\delta_{\mathrm{H}}\left(\mathrm{CHCl}_{3}\right)=7.24, \delta_{\mathrm{H}}\left(\mathrm{C}_{6} \mathrm{D}_{5} \mathrm{H}\right)=7.15, \delta_{\mathrm{C}}\left(\mathrm{CDCl}_{3}\right)=77.00$, $\delta_{\mathrm{C}}\left(\mathrm{C}_{6} \mathrm{D}_{6}\right)=128.00 .{ }^{13} \mathrm{C}$ multiplicities were studied by ATP and/or DEPT and/or HMQC measurements. Mass spectra (EI and HR-EI) were obtained with a spectrometer operated at 70 $\mathrm{eV}$. Optical rotations were measured on a digital polarimeter in a $1 \mathrm{dm}$ cell. Preparative GC was carried out on an instrument employing a thermal conductivity detector, and hydrogen as carrier gas. Analytical GC was performed on an instrument employing a slit/splitless injector, a FID detector, and hydrogen ( 0.6 bar) as carrier gas. The following columns were used: (A): $3 \mathrm{~m} \mathrm{x} \mathrm{1/4"}$ all-glass system, 15\% FFAP on Chromosorb W AW/DMCS 60/80 mesh; (B) 3 x x 1/4" all-glass system, 15\% OV 210 on Chromosorb W AW/DMCS 60/80 mesh; (C) 25 m x 0.25 mm i.d. deactivated fused-silica capillary column coated with oktakis-(2,6-di- $O$-pentyl-3- $O$-butyryl)- $\gamma$ cyclodextrin (Lipodex ${ }^{\circledR}$ E); (D) $25 \mathrm{~m}$ x $0.25 \mathrm{~mm}$ i.d. fused-silica capillary column coated with oktakis-(2,3-di- $O$-pentyl-6- $O$-methyl)- $\gamma$-cyclodextrin $\left(\operatorname{Lipodex}^{\circledR} \mathrm{G}\right.$ ). Product ratios were not 
corrected for relative response. $R_{f}$ values are quoted for Polygram SIL G/UV 254 plates. Colourless substances were detected by oxidation with 3.5\% alcoholic 12-molybdophosphoric acid and subsequent warming. Melting points were observed on a microhotstage and are not corrected. Fresh bakers' yeast was purchased in a normal food store.

\section{Experimental Procedures:}

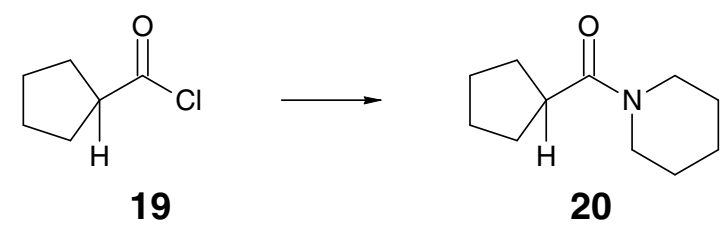

1-(Cyclopentylcarbonyl)-piperidine (20). To a stirred solution of piperidine (32.2 g, $378 \mathrm{mmol})$ in dichloromethane $(140 \mathrm{ml})$ was added under argon with occasional cooling cyclopentanecarboxylic acid chloride $(25.0 \mathrm{~g}, 189 \mathrm{mmol}) \mathrm{such}$, that the internal temperature did not exceed $20^{\circ} \mathrm{C}$. After the addition was complete, the mixture was stirred for $30 \mathrm{~min}$ at room temperature and then washed with water $(80 \mathrm{ml}), 2 \mathrm{~N} \mathrm{HCl}(80 \mathrm{ml})$, saturated sodium carbonate $(80 \mathrm{ml})$ and dried $\left(\mathrm{MgSO}_{4}\right)$. The solvent was distilled off and the residue fractionated to yield $33.0 \mathrm{~g}(96 \%)$ of $\mathbf{2 0}$ as colourless liquid, bp $111^{\circ} \mathrm{C} / 0.35$ torr [purity $>99 \% \mathrm{GC}$, column $\mathrm{A}, 230^{\circ} \mathrm{C}$, retention time (min): 5.82 (20)]. IR (neat): $1640 \mathrm{~cm}^{-1}(\mathrm{C}=\mathrm{O}) ;{ }^{1} \mathrm{H} \mathrm{NMR}\left(500 \mathrm{MHz}, \mathrm{CDCl}_{3}, \mathrm{CHCl}_{3}\right.$ int): $\delta=1.36-1.51(\mathrm{~m}, 6 \mathrm{H}), 1.51-1.58(\mathrm{~m}, 2 \mathrm{H}), 1.58-1.67(\mathrm{~m}, 2 \mathrm{H}), 1.67-1.76(\mathrm{~m}, 4 \mathrm{H}), 2.80(\mathrm{tt}, \mathrm{J}=$ $8 \mathrm{~Hz}, 8 \mathrm{~Hz}, 1 \mathrm{H}), 3.34\left(\mathrm{~m}_{\mathrm{c}}, 2 \mathrm{H}\right), 3.46\left(\mathrm{~m}_{\mathrm{c}}, 2 \mathrm{H}\right) ;{ }^{13} \mathrm{C} \mathrm{NMR}\left(150.8 \mathrm{MHz}, \mathrm{CDCl}_{3}, \mathrm{CDCl}_{3}\right.$ int $): \delta=$ $24.51(\mathrm{t}), 25.45(\mathrm{t}), 25.81(\mathrm{t}), 26.48(\mathrm{t}), 29.92(\mathrm{t}), 40.87(\mathrm{~d}), 42.65(\mathrm{t}), 46.31(\mathrm{t}), 174.01(\mathrm{~s}) ; \mathrm{MS}$ $(\mathrm{EI}): \mathrm{m} / \mathrm{e}=181\left(29, \mathrm{M}^{+}\right), 140(100) ; \mathrm{C}_{11} \mathrm{H}_{19} \mathrm{NO}$ requires $\mathrm{C}, 72.88 ; \mathrm{H}, 10.56$. Found: $\mathrm{C}, 73.11 ; \mathrm{H}$, 10.47 .

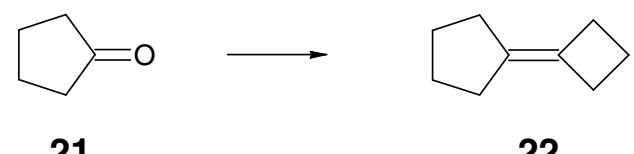

Cyclobutyliden-cyclopentane (22): To a stirred suspension of 4-bromobutyltriphenylphosphonium bromide (765 g, $1.60 \mathrm{~mol}$ ) in dry benzene (1.85 l) was added under argon with stirring potassium t-butoxide ( 3 x $119 \mathrm{~g}, 3.20 \mathrm{~mol}$ ) in $15 \mathrm{~min}$ intervals until the mixture was heated to $50^{\circ} \mathrm{C}$. After $3 \mathrm{~h}$, cyclopentanone $(134 \mathrm{~g}, 1.60 \mathrm{~g})$ was added within $40 \mathrm{~min}$ and after additional 30 
min at $50^{\circ} \mathrm{C}$ the reaction was complete according to $\mathrm{GC}$ [column $\mathrm{B}, 100^{\circ} \mathrm{C}$; retention times (min): 1.63 (cyclopentanone), 2.61 (22)]. The mixture was hydrolyzed with saturated aqueous ammonium chloride $(110 \mathrm{ml})$, the liquid phase was decanted, the residue was extracted with pentane $(3 \times 300 \mathrm{ml})$, and the combined organic phases were concentrated through a $40 \mathrm{~cm}$ vigreux column (bath temperature up to $120^{\circ} \mathrm{C}$ ). The residue was diluted with pentane $(300 \mathrm{ml})$, precipitated triphenylphosphine oxide was filtered off, and the filtrate was washed with water (6 x $200 \mathrm{ml})$ and dried $\left(\mathrm{MgSO}_{4}\right)$. The filtrate was concentrated by distillation over a $20 \mathrm{~cm}$ vigreux column (bath temperature up to $120^{\circ} \mathrm{C}$ ) and the residue fractionated over a microdistillation apparatus to yield $139 \mathrm{~g}(64 \%)$ of 22 as colourless liquid, bp $73-78^{\circ} \mathrm{C} / 30$ torr (purity $90 \% \mathrm{GC}$ ) (lit. ${ }^{1} 155^{\circ} \mathrm{C} / 760$ torr). Under comparable conditions, a second experiment with $1.40 \mathrm{~mol}$ cyclopentanone yielded $121 \mathrm{~g}(67 \%)$ of 22 (purity 94\% GC). For low resolution ${ }^{1} \mathrm{H}$ NMR data, see lit. ${ }^{1}{ }^{1} \mathrm{H}-\mathrm{NMR}\left(500 \mathrm{MHz}, \mathrm{CDCl}_{3}, \mathrm{CHCl}_{3}\right.$ int): $\delta=1.62\left(\mathrm{~m}_{\mathrm{c}}, 4 \mathrm{H}\right), 1.95$ (quint, $\left.\mathrm{J}=8 \mathrm{~Hz}, 2 \mathrm{H}\right)$, $2.04\left(\mathrm{~m}_{\mathrm{c}}, 4 \mathrm{H}\right), 2.57\left(\mathrm{~m}_{\mathrm{c}}, 4 \mathrm{H}\right) ;{ }^{13} \mathrm{C}-\mathrm{NMR}\left(125.7 \mathrm{MHz}, \mathrm{CDCl}_{3}, \mathrm{CDCl}_{3}\right.$ int): $\delta=17.20(\mathrm{t}), 26.80(\mathrm{t})$, $28.94(\mathrm{t}), 29.02(\mathrm{t}), 129.10(\mathrm{~s}), 132.82(\mathrm{~s}) . \mathrm{MS}(\mathrm{EI}): m / e=122\left(36, \mathrm{M}^{+}\right), 93(100)$.

(1) Krapcho, A. P.; Jahngen, Jr., E. G. E. J. Org. Chem. 1974, 39, 1650-1653.

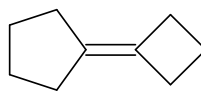

22

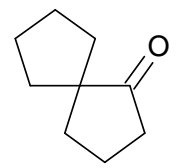

23

Spiro[4.4]nonan-1-one (23). To a vigorously stirred solution of 22 (71.4 g, $585 \mathrm{mmol})$ in dichloromethane (1.50 l) was added 3-chloro-peroxybenzoic acid (156 g, 70\% w/w, $634 \mathrm{mmol})$ such, that the internal temperature did not exceed $30^{\circ}$. Afterwards, $1 \mathrm{~N} \mathrm{KOH} \mathrm{(1.0} \mathrm{1)} \mathrm{was} \mathrm{added}$ and the resulting mixture vigorously stirred until the phases were separated. The aqueous phase was extracted with dichloromethane $(2 \times 300 \mathrm{ml})$, and the combined organic phases were washed with water $(2 \times 200 \mathrm{ml})$ and dried $\left(\mathrm{MgSO}_{4}\right)$. To the filtered solution was added under argon with stirring borontrifluoride etherate $(2.25 \mathrm{~g}, 15.8 \mathrm{mmol}) \mathrm{such}$, that the internal temperature did not exceed $30^{\circ} \mathrm{C}$. With the last drops, the exothermic subsided. The mixture was stirred with solid sodium carbonate $(30 \mathrm{~g})$ until the filtrate was concentrated on a rotary evaporator (bath temperature $50^{\circ} \mathrm{C}$ ) and the residue fractionated over a microdistillation apparatus to yield $73.6 \mathrm{~g}$ (97\%) of 23 as colourless liquid, bp $85-88^{\circ} \mathrm{C} / 16$ torr [purity $98 \% \mathrm{GC}$, column $\mathrm{A}, 160^{\circ} \mathrm{C}$, retention 
time (min): $5.75(23)$ ] (lit $82.5-83^{\circ} \mathrm{C} / 13$ torr, ${ }^{1} 75-76^{\circ} \mathrm{C} / 10$ torr $^{2}$ ). The ${ }^{1} \mathrm{H}$ and ${ }^{13} \mathrm{C}$ NMR data in lit ${ }^{3}$ differ from ours, but the ${ }^{13} \mathrm{C}$ NMR in lit ${ }^{4}$ fit. ${ }^{1} \mathrm{H}$ NMR $\left(500 \mathrm{MHz}, \mathrm{CDCl}_{3}, \mathrm{CHCl}_{3}\right.$ int $): \delta=1.33$ $1.41(\mathrm{~m}, 2 \mathrm{H}), 1.50-1.60(\mathrm{~m}, 2 \mathrm{H}), 1.60-1.73(\mathrm{~m}, 4 \mathrm{H}), 1.73-1.82(\mathrm{~m}, 4 \mathrm{H}), 2.16(\mathrm{t}, \mathrm{J}=7 \mathrm{~Hz}, 2 \mathrm{H})$;

${ }^{13} \mathrm{C} \mathrm{NMR}\left(125.7 \mathrm{MHz}, \mathrm{CDCl}_{3}, \mathrm{CDCl}_{3}\right.$ int): $\delta=19.52(\mathrm{t}), 25.73(\mathrm{t}), 36.24(\mathrm{t}), 37.28(\mathrm{t}), 38.02(\mathrm{t})$, $56.14(\mathrm{~s}), 223.89(\mathrm{~s})$.

(1) Mayer, R.; Wenschuh, G.; Töpelmann, W. Chem. Ber. 1958, 91, 1616-1619.

(2) Krieger, H.; Ruotsalainen, H.; Montin, J. Chem. Ber. 1966, 99, 3715-3717.

(3) Quesnel, Y.; Bidois-Sery, L.; Poirier, J.-M.; Duhamel, L. Synlett 1998, 413-415.

(4) Zimmermann, D. ; Ottinger, R. ; Reisse, J. Org. Magn. Res. 1974, 6, 346-354.

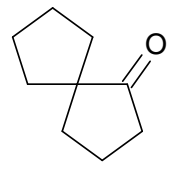

23

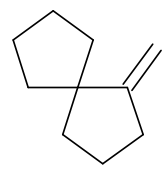

16

1-Methylene-spiro[4.4]nonane (16). To a suspension of methyltriphenylphosphonium bromide $(221 \mathrm{~g}, 619 \mathrm{mmol})$ in anhydrous ether (1.25 l) was added under argon with stirring potassium-tbutoxide $(69.3 \mathrm{~g}, 619 \mathrm{mmol})$ and the mixture heated to reflux. After $30 \mathrm{~min}$, most of the ether was distilled off (bath temperature up to $\left.60^{\circ} \mathrm{C}\right), 23(71.1 \mathrm{~g}, 516 \mathrm{mmol})$ was added, and after additional $1.5 \mathrm{~h}$ at $60^{\circ} \mathrm{C}$ the reaction was complete according to $\mathrm{GC}$ [column $\mathrm{A}, 160^{\circ} \mathrm{C}$, retention times (min): $1.63(\mathbf{1 6}), 5.57(\mathbf{2 3})]$. The mixture was diluted with pentane $(250 \mathrm{ml})$ and hydrolyzed with water $(50 \mathrm{ml})$. The organic phase was decanted, the residue was extracted with pentane $(3 \mathrm{x}$ $250 \mathrm{ml}$ ), and the combined organic phases were washed with water (5 x $250 \mathrm{ml})$ and dried $\left(\mathrm{MgSO}_{4}\right)$. The solvent was distilled off over a $40 \mathrm{~cm}$ vigreux column (bath temperature up to $120^{\circ} \mathrm{C}$ ) and the remaining material fractionated over a microdistillation apparatus to yield $66.3 \mathrm{~g}$ (95\%) of 16 as colourless liquid, bp $79-81^{\circ} \mathrm{C} / 40$ torr (purity $97 \%$ GC) (lit. $162^{\circ} \mathrm{C} / 760$ torr, ${ }^{1}$ $78^{\circ} \mathrm{C} / 25$ torr $\left.^{2}\right)$. The ${ }^{1} \mathrm{H}^{1,3}$ and ${ }^{13} \mathrm{C}$ NMR data ${ }^{3}$ were in accord with literature data.

(1) Christol, H.; Vanel, R. Bull. Soc. Chim. France 1968, 1398-1402.

(2) Cargill, R. L.; Foster, A. M. J. Org. Chem. 1970, 35 1971-1972.

(3) Rieke, R. D.; Xiong, H. Tetrahedron Lett. 1991, 32, 5269-5272. 


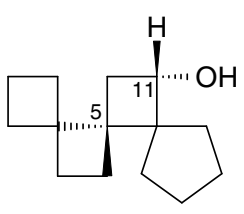

(5S,11S)- 27

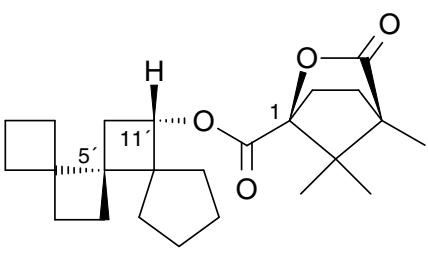

$\left(1 S, 5^{\prime} S, 11^{\prime} S\right)-26$

(1S)-(+)-4,7,7-Trimethyl-3-oxo-2-oxa-bicyclo[2.2.1]heptane-1-carboxylic acid (5'S,11'S)trispiro[3.0.0.4.2.2] tetradecane-11-yl ester [(1S,5'S,11'S)-(+)-26]. To a solution of a 96:4mixture of $(5 S, 11 S)-27$ and $(5 R, 11 S)-28(25 \mathrm{mg}, 0.12 \mathrm{mmol})$ in pyridine $(0.2 \mathrm{ml})$ was added at room temperature under argon with stirring a solution of (1S)-(-)-4,7,7-trimethyl-3-oxo-2-oxabicyclo[2.2.1] heptane-1-carboxylic acid chloride [(1S)-(-)-camphanic acid chloride] (33 mg, 0.14 $\mathrm{mmol})$ in pyridine $(0.2 \mathrm{ml})$. After $2 \mathrm{~h}$ the mixture was diluted with water $(8 \mathrm{ml})$ and extracted with dichloromethane $(3 \times 3 \mathrm{ml})$. The extracts were washed with $2 \mathrm{~N} \mathrm{H}_{2} \mathrm{SO}_{4}(4 \mathrm{ml})$, saturated sodium bicarbonate $(4 \mathrm{ml})$ and dried $\left(\mathrm{MgSO}_{4}\right)$. The solvent was evaporated (bath temperature $40^{\circ} \mathrm{C} / 15$ torr) and the residual solid $\left(42 \mathrm{mg}, \mathrm{mp} 100-105^{\circ} \mathrm{C}\right)$ crystallized from ethanol (1.0 $\left.\mathrm{ml}\right)$ by diffusion of water $(0.6 \mathrm{ml})$ yielding $32 \mathrm{mg}(69 \%)$ of pure $\left(1 S, 5^{\prime} S, 11^{\prime} S\right)-26$ as colourless crystals, $\operatorname{mp} 109-110^{\circ} \mathrm{C}\left([\alpha]_{\mathrm{D}}{ }^{20}=+29.5, \mathrm{c}=1.28\right.$, acetone $)$. IR $(\mathrm{KBr}): 1795,1735 \mathrm{~cm}^{-1}(\mathrm{C}=\mathrm{O}) ;{ }^{1} \mathrm{H}$ NMR $\left(600 \mathrm{MHz}, \mathrm{C}_{6} \mathrm{D}_{6}, \mathrm{C}_{6} \mathrm{D}_{5} \mathrm{H}\right.$ int): $\delta=0.70(\mathrm{~s}, 3 \mathrm{H}), 0.85(\mathrm{~s}, 3 \mathrm{H}), 0.87(\mathrm{~s}, 3 \mathrm{H}), 1.23\left(\mathrm{~m}_{\mathrm{c}}, 2 \mathrm{H}\right), 1.36\left(\mathrm{~m}_{\mathrm{c}}\right.$, $1 \mathrm{H}), 1.48-1.56(\mathrm{~m}, 4 \mathrm{H}), 1.58-1.74(\mathrm{~m}, 7 \mathrm{H}), 1.75-1.84(\mathrm{~m}, 2 \mathrm{H}), 1.88\left(\mathrm{~m}_{\mathrm{c}}, 1 \mathrm{H}\right), 1.98-2.11(\mathrm{~m}, 5 \mathrm{H})$, $2.25\left(\mathrm{~m}_{\mathrm{c}}, 1 \mathrm{H}\right), 4.83(\mathrm{dd}, \mathrm{J}=8 \mathrm{~Hz}, 7.5 \mathrm{~Hz}, 1 \mathrm{H}) ;{ }^{13} \mathrm{C} \mathrm{NMR}\left(150.8 \mathrm{MHz}, \mathrm{C}_{6} \mathrm{D}_{6}, \mathrm{C}_{6} \mathrm{D}_{6}\right.$ int $): \delta=9.80$ (q), $15.85(\mathrm{t}), 16.51(\mathrm{q}), 16.80(\mathrm{q}), 24.88(\mathrm{t}), 26.08(\mathrm{t}), 26.13(\mathrm{t}), 27.15(\mathrm{t}), 28.78(\mathrm{t}), 30.36(\mathrm{t})$, $30.76(\mathrm{t}), 30.99(\mathrm{t}), 32.68(\mathrm{t}), 35.40(\mathrm{t}), 35.80(\mathrm{t}), 46.10(\mathrm{~s}), 48.76(\mathrm{~s}), 53.79(\mathrm{~s}), 54.62(\mathrm{~s}), 57.63$ (s), 74.80 (d), 90.70 (s), 167.37 (s), 177.32 (s); MS (EI): m/e = $386\left(<1, \mathrm{M}^{+}\right), 134$ (100) ; MS (HR-EI): calcd for $\mathrm{C}_{24} \mathrm{H}_{34} \mathrm{O}_{4}$ : 386.2457; found 386.2457.

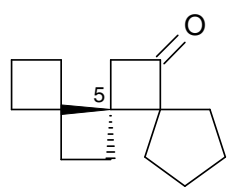

(5R)-15

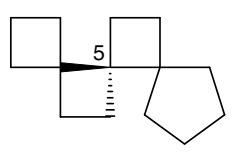

$(5 R)-6$

(5R)-(-)-Trispiro[3.0.0.4.2.2]tetradecane [(5R)-6]. (5R)-15 (111 $\mathrm{mg}, 0.54 \mathrm{mmol})$ was deoxigenated as described for the racemic material yielding $69 \mathrm{mg}(67 \%)$ of crude $(5 R)-6$ (purity 97\% GC). Preparative gas chromatography [column A, 180 ${ }^{\circ}$, retention time (min): 3.69] yielded 
analytically pure $(5 R)-6$ as colourless liquid (purity $>99.5 \%$ GC). According to capillary gas chromatography on a chiral phase [column $\mathrm{D}, 90^{\circ} \mathrm{C}$, retention time (min): 33.93 ] the material was enantiomerically pure $\left(>99 \%\right.$ ee; $\left.[\alpha]_{\mathrm{D}}{ }^{20}=-4.5, \mathrm{c}=1.13, \mathrm{CHCl}_{3}\right)$. The ${ }^{1} \mathrm{H}$ and ${ }^{13} \mathrm{C}$ NMR data were identical with those of racemic 6.

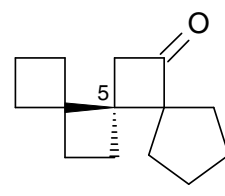

$(5 R)-15$

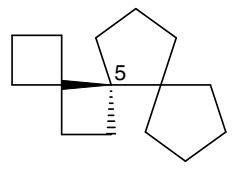

(5S)-7

(5S)-(-)-Trispiro[3.0.0.4.3.2]pentadecane [(5S)-7]. (5R)-15 (204 $\mathrm{mg}, 1.00 \mathrm{mmol})$ was ring enlarged as described for the racemic material yielding $186 \mathrm{mg}(85 \%)$ of a 55:45-mixture of $(5 R)$ $\mathbf{2 4}$ and (5R)-25 (purity 93\% GC). This material was deoxygenated as described for the racemic mixture yielding $103 \mathrm{mg}$ (59\%) of crude (5S)-7 (purity 84\% GC). Preparative gas chromatography [column $\mathrm{A}, 180^{\circ} \mathrm{C}$, retention time (min): 6.21 ] yielded analytically pure (5S)-7 as colourless liquid (purity $>99.5 \% \mathrm{GC}$ ). As derived from $(5 R)-15$ ( $>99 \%$ ee), (5S)-7 was enantiomerically pure $\left(>99 \%\right.$ ee; $\left.[\alpha]_{\mathrm{D}}{ }^{20}=-1.1, \mathrm{c}=1.10, \mathrm{CHCl}_{3}\right)$. The ${ }^{1} \mathrm{H}$ and ${ }^{13} \mathrm{C}$ NMR data were identical with those of racemic 7 .

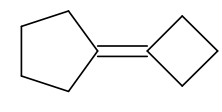

22

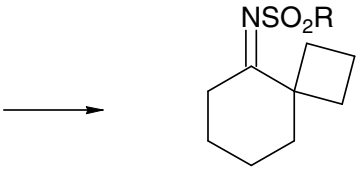

$29 \mathrm{R}=4-\mathrm{NO}_{2} \mathrm{C}_{6} \mathrm{H}_{4}$

5-(4-Nitrobenzenesulfonimido)spiro[3.5]nonane (29): Protected from light, a stirred solution of 4-nitrobenzenesulfonic acid azide ${ }^{1}(311 \mathrm{~g}, 1.36 \mathrm{~mol})$ and 22 (140 g, $1.14 \mathrm{~mol}$, purity 90\%) in anhydrous acetonitrile (1.40 l) was heated under argon to reflux. After $20 \mathrm{~h}, \mathrm{TLC}\left[\mathrm{CH}_{2} \mathrm{Cl}_{2}, \mathrm{R}_{\mathrm{f}}=\right.$ 0.88 (22), 0.64 (29)] indicated that the reaction was complete. The mixture was cooled and crystallized for $24 \mathrm{~h}$ at $-18^{\circ} \mathrm{C}$ yielding a first crop of $188 \mathrm{~g} \mathrm{(57 \% )}$ of 29 as pale yellow crystals, $\mathrm{mp} 127-129^{\circ} \mathrm{C}\left(\right.$ lit. $\left.^{2} 131^{\circ} \mathrm{C}\right)$. The filtrate was concentrated and the residue $(204 \mathrm{~g})$ was dissolved in acetonitrile $(300 \mathrm{ml})$ and crystallized for $72 \mathrm{~h}$ at $-18^{\circ} \mathrm{C}$ yielding a second crop of $41 \mathrm{~g}(12 \%)$ of 29 as pale yellow crystals, mp $133-135^{\circ} \mathrm{C}$. Combined yield $229 \mathrm{~g}(69 \%)$. The ${ }^{1} \mathrm{H}$ and ${ }^{13} \mathrm{C}$ NMR data were in accord with literature data. ${ }^{2}$ 
(1) Horner, L.; Christmann, A. Chem. Ber. 1963, 96, 388-398.

(2) Fitjer, L.; Rissom, B.; Kanschik, A.; Egert, E. Tetrahedron 1994, 50, 10879-10892.

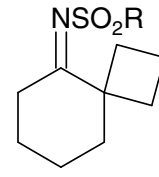

$29 \mathrm{R}=4-\mathrm{NO}_{2} \mathrm{C}_{6} \mathrm{H}_{4}$

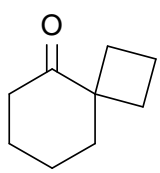

30

Spiro[3.5]nonan-5-one (30): 29 (229 g, $0.71 \mathrm{~mol})$ was saponified in a 1:1-mixture of ethanol and 4N aqueous $\mathrm{HCl}$ as described in lit. ${ }^{1}$ According to TLC $\left[\mathrm{CH}_{2} \mathrm{Cl}_{2}, \mathrm{R}_{\mathrm{f}}=0.64(\mathbf{2 9}), 0.54(\mathbf{3 0})\right]$ after $2 \mathrm{~h}$ at $50^{\circ} \mathrm{C}$ the reaction was complete (lit. $\left.16 \mathrm{~h}\right)$. Work up and distillation yielded $74 \mathrm{~g}(71 \%)$ of 30 as colourless liquid, bp $82-88^{\circ} \mathrm{C} / 18$ torr (lit. $78-83 / 10$ torr). According to GC [column A, $160^{\circ} \mathrm{C}$; retention times (min): $6.09(30)$ ], the material was $98 \%$ pure. The ${ }^{1} \mathrm{H}$ and ${ }^{13} \mathrm{C}$ NMR data were in accord with literature data. ${ }^{1}$

(1) Fitjer, L.; Rissom, B.; Kanschik, A.; Egert, E. Tetrahedron 1994, 50, 10879-10892.

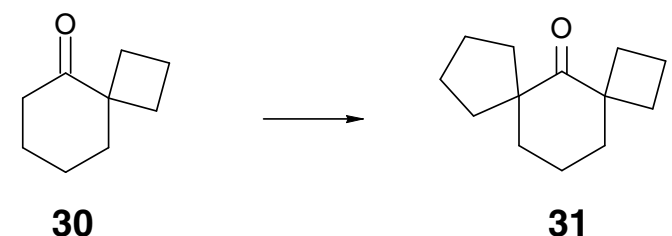

Dispiro[3.1.4.3]tridecan-5-one (31): To a suspension of potassium hydride $(28.7 \mathrm{~g}, 251 \mathrm{mmol})$ in ether $(300 \mathrm{ml})$ was added at room temperature under argon with stirring within $1.5 \mathrm{~h} \mathbf{3 0}$ (11.7 g, $85 \mathrm{mmol}$ ). After additional $40 \mathrm{~min}$ the hydrogen evolution had ceased. 1,4-dibromobutane $(21.7 \mathrm{~g}, 100 \mathrm{mmol})$ was added and the resulting mixture stirred at room temperature. After $24 \mathrm{~h}$, $\mathrm{GC}$ analysis [column $\mathrm{A}, 180^{\circ} \mathrm{C}$; retention times (min): 3.59 (30), 3.82 (1,4-dibromobutane), 9.61 (31)] revealed, that $35 \%$ of unreacted 30 were still present. Additional 1,4-dibromobutane $(6.25$ $\mathrm{g}, 29 \mathrm{mmol}$ ) was added, and after a total of $90 \mathrm{~h}$ the reaction was complete. The mixture was diluted with pentane $(100 \mathrm{ml})$ and hydrolyzed with saturated aqueous ammonium chloride (12 $\mathrm{ml})$. The organic phase was decanted, the residue was extracted with pentane $(3 \times 30 \mathrm{ml})$, and the combined organic phases were dried $\left(\mathrm{MgSO}_{4}\right)$ and concentrated on a rotary evaporator (bath temperature $45^{\circ} \mathrm{C} / 20$ torr). The remaining material $(20.6 \mathrm{~g}$ ) was distilled to yield $12.5 \mathrm{~g}$ (76\%) of 
31 as colourless liquid, bp $104-113^{\circ} \mathrm{C} / 1.3$ torr (purity $98 \% \mathrm{GC}$ ). IR (neat): $1695 \mathrm{~cm}^{-1}(\mathrm{C}=\mathrm{O})$; ${ }^{1} \mathrm{H}-$ NMR (600 MHz, $\mathrm{CDCl}_{3}, \mathrm{CHCl}_{3}$ int): $\delta=1.28-1.35$ (m, 2H), 1.50-1.62 (m, 6H), 1.62-1.74 (m, 5H), 1.86-1.95 (m, 3H), 1.95-2.02 (m, 2H), 2.43-2.50 (m, 2H); ${ }^{13} \mathrm{C}-\mathrm{NMR}\left(75.5 \mathrm{MHz}, \mathrm{CDCl}_{3}\right.$, $\mathrm{CDCl}_{3}$ int): $\delta=15.38(\mathrm{t}), 19.28(\mathrm{t}), 24.67(\mathrm{t}), 29.93(\mathrm{t}), 35.97(\mathrm{t}), 37.62(\mathrm{t}), 38.71(\mathrm{t}), 50.50(\mathrm{~s})$, 56.13 (s), 215.33 (s). MS (EI): $m / e=192\left(5, \mathrm{M}^{+}\right), 41$ (100). $\mathrm{C}_{13} \mathrm{H}_{20} \mathrm{O}$ requires $\mathrm{C}, 81.20 ; \mathrm{H}, 10.48$. Found: C, 80.88; H, 10.26.

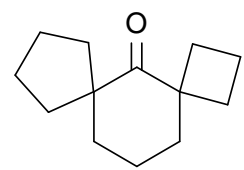

31

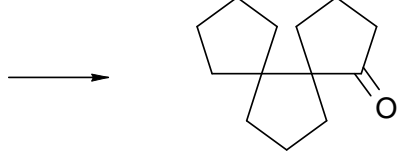

32

Dispiro[4.0.4.3]tridecan-1-one (32): A stirred solution of $\mathbf{3 1}(12.5 \mathrm{~g}, 65 \mathrm{mmol})$ in formic acid $(125 \mathrm{ml})$ was heated under argon to $50-55^{\circ} \mathrm{C}$ until $\mathrm{GC}$ analysis [column $\mathrm{A}, 230^{\circ} \mathrm{C}$; retention times (min): 5.37 (31), 6.01 (32)] indicated, that 31 had been consumed (26 h). The mixture was poured into ice water $(750 \mathrm{ml})$ and extracted with pentane $(5 \times 150 \mathrm{ml})$. The combined extracts were washed with water $(100 \mathrm{ml})$, dried $\left(\mathrm{Na}_{2} \mathrm{CO}_{3}\right)$ and concentrated on a rotary evaporator (bath temperature $40^{\circ} \mathrm{C} / 20$ torr). The residue (11.5 g brown oil) was chromatographed on silica gel $(0.05-0.20 \mathrm{~mm})$ in pentane/ether 9:1 [column $75 \times 5.5 \mathrm{~cm}, \mathrm{R}_{\mathrm{f}}=0.34(\mathbf{3 2})$ ] to yield $4.64 \mathrm{~g}(38 \%)$ of 32 as colourless liquid (purity $99 \%$ GC). The ${ }^{1} \mathrm{H}$ and ${ }^{13} \mathrm{C}$ NMR data were in accord with literature data. ${ }^{1}$

(1) Kakiuchi, K.; Okada, H.; Kanehisa, N.; Kai, Y.; Kurosawa, H. J. Org. Chem. 1996, 61, 2972-2979.

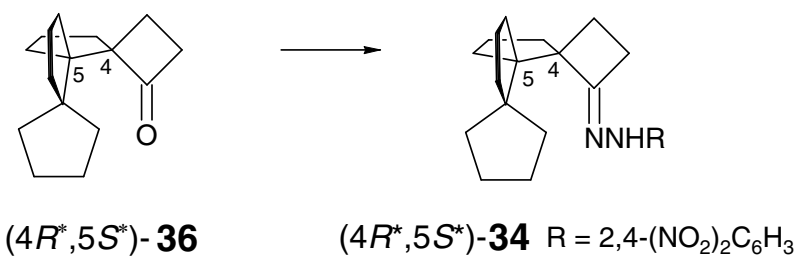

$\left(4 R^{*}, 5 S^{*}\right)$-N-Trispiro[3.0.0.4.3.3]hexadec-1-ylidene-N'-2,4-dinitrophenyl-hydrazine

[(4R*,5 $\left.\left.S^{*}\right)-34\right]:$ 2,4-Dinitrophenylhydrazine (100 $\left.\mathrm{mg}, 0.50 \mathrm{mmol}\right)$ was dissolved in a mixture of concentrated sulphuric acid $(0.50 \mathrm{ml})$, water $(0.75 \mathrm{ml})$ and ethanol $(2.5 \mathrm{ml})$. $36(50 \mathrm{mg}, 0.22$ 
mmol) was added and after 15 min stirring the clear solution was allowed to stand for 7 days at room temperature. The precipitated orange crystals of $\mathbf{3 4}$ were filtered off, washed with water (5 x $1 \mathrm{ml})$, acetone ( $0.5 \mathrm{ml})$ and dried. Yield: $58 \mathrm{mg}(63 \%), \mathrm{mp} 152^{\circ} \mathrm{C}$. IR (KBr): $3330(\mathrm{~N}-\mathrm{H}), 1625$ $\mathrm{cm}^{-1}(\mathrm{C}=\mathrm{N}) ;{ }^{1} \mathrm{H}-\mathrm{NMR}\left(600 \mathrm{MHz}, \mathrm{CDCl}_{3}, \mathrm{CHCl}_{3}\right.$ int $): \delta=1.24-1.36(\mathrm{~m}, 3 \mathrm{H}), 1.39-1.74(\mathrm{~m}, 12 \mathrm{H})$, 1.76-1.90 (m, 3H), 2.02-2.13 (m, 3H), 2.41 (ddd, J = 12, 10, 7 Hz, 1H), 2.77-2.89 (m 2H), 7.78 $(\mathrm{d}, \mathrm{J}=9.5 \mathrm{~Hz}, 1 \mathrm{H}), 8.25(\mathrm{dd}, \mathrm{J}=9.5,2.5 \mathrm{~Hz}, 1 \mathrm{H}), 9.08(\mathrm{~d}, \mathrm{~J}=2.5 \mathrm{~Hz}, 1 \mathrm{H}), 10.67(\mathrm{br} \mathrm{s}, 1 \mathrm{H}) ;{ }^{13} \mathrm{C}-$ NMR (150.8 MHz, $\mathrm{CDCl}_{3}, \mathrm{CDCl}_{3}$ int): $\delta=19.74(\mathrm{t}), 20.33(\mathrm{t}), 22.99(\mathrm{t}), 24.09$ (t), $24.19(\mathrm{t})$, $25.70(\mathrm{t}), 34.36(\mathrm{t}), 34.99(\mathrm{t}), 35.06(\mathrm{t}), 35.71(\mathrm{t}), 39.58(\mathrm{t}), 41.01(\mathrm{t}), 56.09(\mathrm{~s}), 58.50(\mathrm{~s}), 63.48$ (s), 116.03 (d), 123.63 (d), 128.65 (s), 130.05 (d), 137.35 (s), 144.91 (s), 169.40 (s); MS (EI): m/e $=412\left(17, \mathrm{M}^{+}\right), 263(100) . \mathrm{C}_{22} \mathrm{H}_{28} \mathrm{~N}_{4} \mathrm{O}_{4}$ requires $\mathrm{C}, 64.06 ; \mathrm{H}, 6.84$. Found: $\mathrm{C}, 64.32 ; \mathrm{H}, 6.64$.

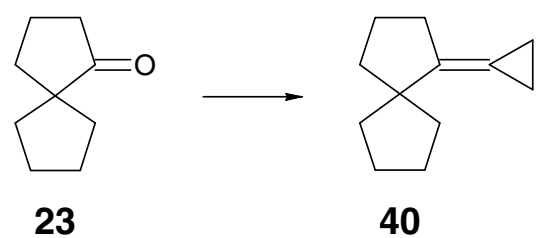

Cyclopropyliden-spiro[4.4]nonane (40): To a suspension of sodium hydride (10.1 g, $0.42 \mathrm{~mol})$ in anhydrous THF $(600 \mathrm{ml})$ was added under argon with stirring cyclopropyltriphenylphosphonium bromide $^{1}(161 \mathrm{~g}, 0.42 \mathrm{~mol})$ and the mixture heated to $70^{\circ} \mathrm{C}$. After $4 \mathrm{~h}, 22(41.7 \mathrm{~g}, 0.30$ mol) was added and the reaction progress monitored by $\mathrm{GC}$ [column $\mathrm{A}, 140^{\circ} \mathrm{C}$; retention times (min): $8.21(\mathbf{4 0}), 10.97(\mathbf{2 3})]$. After $50 \mathrm{~h}$ at $70^{\circ} \mathrm{C}$, the reaction had ceased. The mixture was poured into water $(700 \mathrm{ml})$ and extracted with pentane $(4 \times 300 \mathrm{ml})$, and the combined organic phases were washed with water $(2 \times 200 \mathrm{ml})$ and dried $\left(\mathrm{MgSO}_{4}\right)$. Most of the solvent was distilled off over a $30 \mathrm{~cm}$ vigreux column with occasional filtration from precipitated triphenylphosphine oxide (bath temperature up to $120^{\circ} \mathrm{C}$ ), and the residue was fractionated at reduced pressure to yield $28.9 \mathrm{~g}(52 \%)$ of $\mathbf{4 0}$ as colourless liquid, bp $85-95^{\circ} \mathrm{C} / 14$ torr (purity $88 \% \mathrm{GC}$ ). This material was used in the next step. A pure sample was obtained by preparative GC. ${ }^{1} \mathrm{H}-\mathrm{NMR}$ (600 MHz, $\mathrm{C}_{6} \mathrm{D}_{6}, \mathrm{C}_{6} \mathrm{D}_{5} \mathrm{H}$ int): $\delta=0.80$ ( $\mathrm{AA}^{\prime}$-part of a $\mathrm{AA}^{\prime} \mathrm{BB}^{\prime}$-system, $2 \mathrm{H}$ ), 1.03 (BB'-part of a AA'BB'-system, 2H), 1.48-1.54 (m, 2H), 1.54-1.59 (m, 2H), 1.59-1.66 (m, 4H), 1.70-1.79 (m, $2 \mathrm{H}), 1.85-1.92(\mathrm{~m}, 2 \mathrm{H}), 2.49\left(\mathrm{~m}_{\mathrm{c}}, 2 \mathrm{H}\right) ;{ }^{13} \mathrm{C}-\mathrm{NMR}\left(150.8 \mathrm{MHz}, \mathrm{C}_{6} \mathrm{D}_{6}, \mathrm{C}_{6} \mathrm{D}_{6}\right.$ int $): \delta=-0.2(\mathrm{t}), 2.4$ (t), $24.3(\mathrm{t}), 25.5(\mathrm{t}), 33.3(\mathrm{t}), 39.4(\mathrm{t}), 41.8(\mathrm{t}), 54.0(\mathrm{~s}), 108.8(\mathrm{~s}), 138.9(\mathrm{~s}) ; \mathrm{MS}(\mathrm{EI}): \mathrm{m} / \mathrm{e}=162$ $\left(4, \mathrm{M}^{+}\right), 91$ (100). $\mathrm{C}_{13} \mathrm{H}_{20} \mathrm{O}$ requires $\mathrm{C}, 88.82 ; \mathrm{H}, 11.18$. Found: C, 88.71; H, 11.20. 
(1) Utimoto, K.; Tamura, M.; Sisido, K. Tetrahedron 1973, 29, 1169-1171.

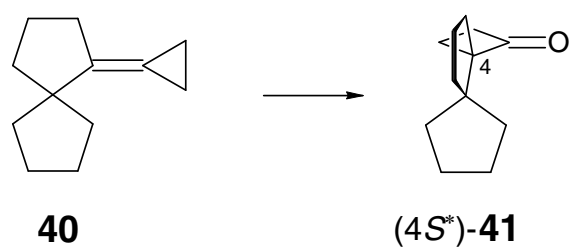

(4S*)-Dispiro[3.0.4.3]dodecan-1-one [(4S*)-41]: To a stirred solution of 40 (28.9 g, $178 \mathrm{mmol}$, purity $88 \%)$ in dichloromethane $(400 \mathrm{ml})$ was added 3-chloroperoxybenzoic acid $(53.7 \mathrm{~g}, 70 \%$ w/w, $217 \mathrm{mmol})$ such, that the internal temperature did not exceed $30^{\circ} \mathrm{C}(1.5 \mathrm{~h})$. According to TLC [pentane/ether 9:1; $\mathrm{R}_{\mathrm{f}}=0.80(\mathbf{4 0}), 0.31$ (epoxide)], after additional $45 \mathrm{~min}$ the reaction was complete. $1 \mathrm{~N} \mathrm{KOH}(300 \mathrm{ml})$ was added and the resulting mixture vigorously stirred until the phases were separated. The organic phase was washed with water $(2 \times 250 \mathrm{ml})$ and dried $\left(\mathrm{MgSO}_{4}\right)$. A part of the solvent was distilled off and the remaining solution (300 $\left.\mathrm{ml}\right)$ cooled to room temperature until borontrifluoride etherate $(0.50 \mathrm{ml})$ was added dropwise such, that the internal temperature did not exceed $30^{\circ} \mathrm{C}$. With the last drops, the exothermic reaction subsided and TLC [pentane/ether 9:1; $\mathrm{R}_{\mathrm{f}}=0.43$ (41), 0.31 (epoxide)] indicated, that the rearrangement was complete. The mixture was stirred with solid sodium carbonate (15 g), the filtrate was concentrated (bath temperature $40^{\circ} \mathrm{C} / 15$ torr), and the residue was fractionated over a microdistillation apparatus to yield $16.4 \mathrm{~g}(51 \%)$ of $\mathbf{4 1}$ as colourless liquid, bp 110-119/12 torr (purity $87 \%$ GC). This material was used in the next step. A pure sample was obtained by preparative $\mathrm{GC}$ [column $\mathrm{B}, 200^{\circ} \mathrm{C}$; retention time (min): $2.56(\mathbf{4 1})$ ]. IR (neat): $1770 \mathrm{~cm}^{-1}(\mathrm{C}=\mathrm{O})$; ${ }^{1} \mathrm{H}-\mathrm{NMR}\left(600 \mathrm{MHz}, \mathrm{CDCl}_{3}, \mathrm{CHCl}_{3}\right.$ int): $\delta=1.29-1.34$ (m, 2H), 1.36-1.42 (m, 1H), 1.45-1.70 (m, $10 \mathrm{H}), 1.74(\mathrm{ddd}, \mathrm{J}=11,10.5,6.5 \mathrm{~Hz}, 1 \mathrm{H}), 1.96(\mathrm{ddd}, \mathrm{J}=11,11,7 \mathrm{~Hz}, 1 \mathrm{H}), 1.99-2.05$ (m, 1H), 2.69 (ddd, J = 17.5, 11, $6.5 \mathrm{~Hz}, 1 \mathrm{H}), 2.87$ (ddd, J = 17.5, 10.5, $7 \mathrm{~Hz}, 1 \mathrm{H}) ;{ }^{13} \mathrm{C}-\mathrm{NMR}(150.8 \mathrm{MHz}$, $\mathrm{CDCl}_{3}, \mathrm{CDCl}_{3}$ int): $\delta=19.2(\mathrm{t}), 20.5(\mathrm{t}), 24.3(\mathrm{t}), 25.0(\mathrm{t}), 33.3(\mathrm{t}), 33.8(\mathrm{t}), 34.2(\mathrm{t}), 37.6(\mathrm{t}), 42.3$ (t), 54.5 (s), 76.3 (s), 216.5 (s): MS (EI): $m / e=178\left(20, \mathrm{M}^{+}\right), 122$ (100). $\mathrm{C}_{12} \mathrm{H}_{18} \mathrm{O}$ requires $\mathrm{C}$, 80.85; H, 10.18. Found: C, 81.12; H, 9.89. 


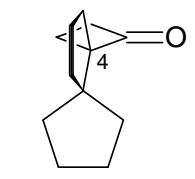

$\left(4 S^{*}\right)-41$

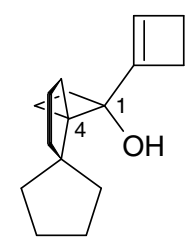

$\left(1 R^{\star}, 4 S^{\star}\right)-44$

$\left(1 R^{*}, 4 S^{*}\right)-1-C y c l o b u t-1-e n y l-d i s p i r o[3.0 .4 .3]$ dodecan-1-ol [ $\left.\left(1 R^{*}, 4 S^{*}\right)-44\right]$ : To a solution of 1bromo-cyclobutene $^{1}$ (4.99 g, $\left.37.5 \mathrm{mmol}\right)$ in anhydrous THF $(125 \mathrm{ml})$ was added within $1 \mathrm{~h}$ at $78^{\circ} \mathrm{C}$ under argon with stirring a 1.5-molar solution of tert-butyllithium in pentane $(50 \mathrm{ml}, 75$ mmol). After additional $45 \mathrm{~min}$ at $-78^{\circ} \mathrm{C}$, the mixture was allowed to warm to $0^{\circ} \mathrm{C}$. The resulting solution of 1-lithio-cyclobutene was used in the next step. A suspension of finely powdered dry $\mathrm{CeCl}_{3}(7.38 \mathrm{~g}, 30 \mathrm{mmol})$ in anhydrous THF $(110 \mathrm{ml})$ was stirred at room temperature under argon for $2 \mathrm{~h}$. After addition of 41 (2.67 g, $15 \mathrm{mmol}$, purity 87\%), stirring was continued for $2 \mathrm{~h}$ until the mixture was cooled to $0^{\circ} \mathrm{C}$ and the solution of 1-lithio-cyclobutene added within 25 min. According to $\mathrm{GC}$ [column $\mathrm{B}, 200^{\circ} \mathrm{C}$; retention time (min): 2.56 (41), 3.97 (44)], after $1 \mathrm{~h}$ the reaction was complete. The mixture was diluted with pentane $(150 \mathrm{ml})$ and hydrolyzed with saturated aqueous ammonium chloride $(40 \mathrm{ml})$. The liquid phase was decanted, the residue was extracted with pentane $(2 \times 75 \mathrm{ml})$, and the combined organic phases were washed with water $(2$ x $150 \mathrm{ml}$ ) and dried $\left(\mathrm{MgSO}_{4}\right)$. The solvents were distilled off (bath temperature $40^{\circ} \mathrm{C} / 15$ torr) and the residual yellow liquid (4.0 g) chromatographed on silica gel $(0.05-0.20 \mathrm{~mm})$ in pentane/ether 9:1 [column 75 × $6 \mathrm{~cm}, \mathrm{R}_{\mathrm{f}}=0.43(\mathbf{4 1}), 0.39(\mathbf{4 4})$ ] yielding $2.02 \mathrm{~g}(64 \%)$ of 44 as colourless liquid (purity 96\% GC). IR (neat): $3600(\mathrm{OH}), 3580-3300 \mathrm{~cm}^{-1}\left(\mathrm{OH}_{\mathrm{ass}}\right) ;{ }^{1} \mathrm{H}-\mathrm{NMR}(600$ $\mathrm{MHz}, \mathrm{C}_{6} \mathrm{D}_{6}, \mathrm{C}_{6} \mathrm{D}_{5} \mathrm{H}$ int): $\delta=1.07$ (br s, $\left.1 \mathrm{H}\right), 1.30-1.41$ (m, 4H), 1.46-1.65 (m, 7H), 1.72-1.85 (m, $4 \mathrm{H}), 2.10-2.20(\mathrm{~m}, 2 \mathrm{H}), 2.23\left(\mathrm{~m}_{\mathrm{c}}, 2 \mathrm{H}\right), 2.36-2.50(\mathrm{~m}, 3 \mathrm{H}), 5.67\left(\mathrm{~m}_{\mathrm{c}}, 1 \mathrm{H}\right) ;{ }^{13} \mathrm{C}-\mathrm{NMR}(150.8 \mathrm{MHz}$, $\mathrm{C}_{6} \mathrm{D}_{6}, \mathrm{C}_{6} \mathrm{D}_{6}$ int): $\delta=19.48(\mathrm{t}), 25.40(\mathrm{t}), 25.49(\mathrm{t}), 25.76(\mathrm{t}), 26.32(\mathrm{t}), 29.98(\mathrm{t}), 30.11(\mathrm{t}), 34.08$ $(\mathrm{t}), 35.34(\mathrm{t}), 36.52(\mathrm{t}), 38.84(\mathrm{t}), 55.07(\mathrm{~s}), 58.02(\mathrm{~s}), 79.97(\mathrm{~s}), 127.86(\mathrm{~d}), 153.71(\mathrm{~s})$; MS (EI): $m / e=232\left(2, \mathrm{M}^{+}\right), 96(100)$. HRMS $m / e\left(\mathrm{M}^{+}\right)$calcd 232.1827, obsd 232.1827. $\mathrm{C}_{16} \mathrm{H}_{24} \mathrm{O}$ requires C, 82.70; H, 10.41. Found: C, 81.75; H, 10.14.

(1) Abell, P. I.; Chiao, C. J. Am. Chem. Soc. 1960, 82, 3610-3613. 


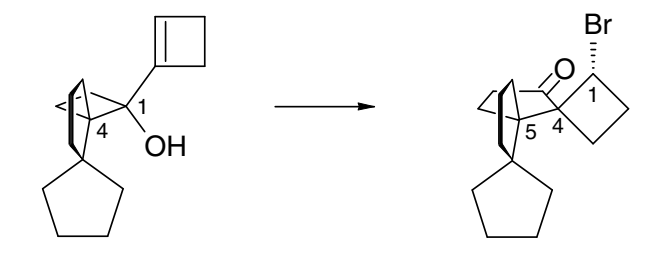

$\left(1 R^{\star}, 4 S^{\star}\right)-44$

$\left(1 R^{\star}, 4 S^{\star}, 5 S^{\star}\right)-45$

$\left(1 R^{*}, 4 S^{*}, 5 S^{*}\right)-1-B r o m o-t r i s p i r o[3.0 .0 .4 .3 .3]$ hexadecane-16-one $\left[\left(1 R^{*}, 4 S^{*}, 5 S^{*}\right)-45\right]$ : To dry zinc bromide $(2.10 \mathrm{~g}, 9.31 \mathrm{mmol})$ in dry acetonitrile $(42 \mathrm{ml})$ was added dry N-chloro-toluene-4sulfonamide $(2.12 \mathrm{~g}, 9.31 \mathrm{mmol})$ and the mixture stirred under argon until a nearly clear yellow solution was formed (10 min). This solution was added under argon with stirring to neat 44 (1.78 $\mathrm{g}, 7.67 \mathrm{mmol})$. After $1.5 \mathrm{~h}$, TLC [pentane/ether 95:5; $\mathrm{R}_{\mathrm{f}}=0.25(\mathbf{4 4}), 0.23(\mathbf{4 5})$ ] indicated that the reaction was complete. The mixture was filtered over a short path of silica gel (colum 21 x 4.5 $\mathrm{cm}$ ) and eluted with ether. The solvents were evaporated (bath temperature $40^{\circ} \mathrm{C} / 15$ torr) and the heterogeneous residue $(3.8 \mathrm{~g})$ extracted with pentane $(5 \times 25 \mathrm{ml})$. The extracts were concentrated (bath temperature $40^{\circ} \mathrm{C} / 15$ torr) and the residue (2.36 g) chromatographed on silica gel (0.05-0.20 $\mathrm{mm}$ ) in pentane/ether 95:5 (column 75 x $5.5 \mathrm{~cm}$ ) to yield $1.58 \mathrm{~g}(66 \%)$ of 45 as colourless oil (purity 90\% NMR), which solidified on cooling; mp $29^{\circ} \mathrm{C}$. IR (neat): $1738 \mathrm{~cm}^{-1}(\mathrm{C}=\mathrm{O}) ;{ }^{1} \mathrm{H}-\mathrm{NMR}$ (600 MHz, $\mathrm{C}_{6} \mathrm{D}_{6}, \mathrm{C}_{6} \mathrm{D}_{5} \mathrm{H}$ int): $\delta=0.91-0.99$ (m, 2H), 0.99-1.14 (m, 2H), 1.18-1.41 (m, 9H), 1.52$1.63(\mathrm{~m}, 4 \mathrm{H}), 1.92-2.02(\mathrm{~m}, 2 \mathrm{H}), 2.26\left(\mathrm{~m}_{\mathrm{c}}, 1 \mathrm{H}\right), 2.35\left(\mathrm{~m}_{\mathrm{c}}, 1 \mathrm{H}\right), 2.62\left(\mathrm{~m}_{\mathrm{c}}, 1 \mathrm{H}\right), 4.20(\mathrm{dd}, \mathrm{J}=9,9$ $\mathrm{Hz}, 1 \mathrm{H}) ;{ }^{13} \mathrm{C}-\mathrm{NMR}\left(150.8 \mathrm{MHz}, \mathrm{C}_{6} \mathrm{D}_{6}, \mathrm{C}_{6} \mathrm{D}_{6}\right.$ int): $\delta=19.72(\mathrm{t}), 22.69(\mathrm{t}), 23.05(\mathrm{t}), 25.31(\mathrm{t})$, $29.22(\mathrm{t}), 29.62(\mathrm{t}), 33.53(\mathrm{t}), 34.68(\mathrm{t}), 34.91(\mathrm{t}), 36.30(\mathrm{t}), 37.28(\mathrm{t}), 44.70(\mathrm{~d}), 55.17(\mathrm{~s}), 56.85$ (s), 65.63 (s), 215.24 (s); MS (EI): m/e = 312, $310\left(8,8, \mathrm{M}^{+}\right), 231$ (100). HRMS m/e (M+) calcd 310.0932, obsd 310.0932. $\mathrm{C}_{16} \mathrm{H}_{23} \mathrm{BrO}$ requires $\mathrm{C}, 61.74 ; \mathrm{H}, 7.45$. Found: $\mathrm{C}, 61.96 ; \mathrm{H}, 7.23$.

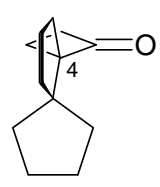

$\left(4 S^{\star}\right)-41$

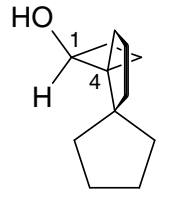

$(1 S, 4 R)-51$

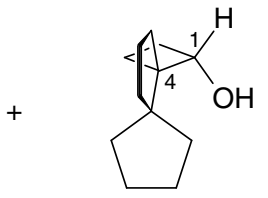

$(1 S, 4 S)-53$

$(1 S, 4 R)-(+)$-Dispiro[3.0.4.3]dodecan-1-ol $\quad[(1 S, 4 R)-(+)-51] \quad$ and $\quad(1 S, 4 S)-(+)$-Dispiro[3.0.4.3]dodecan-1-ol [(1S,4S)-(+)-53]: To (-)-diisopinocampheylchloroborane [(-)-DIP-Cl] (21.6 g, $67.4 \mathrm{mmol})$ was added within $15 \mathrm{~min}$ under argon with stirring rac-41 (10.0 g, 56.2 
mmol, purity 97\%). A slightly exothermic reaction occurred and a clear solution formed. According to $\mathrm{GC}$ analyses [column A, $230^{\circ} \mathrm{C}$; retention times (min): $3.71(\mathbf{4 1}), 5.40(\mathbf{5 1}, \mathbf{5 3})$ ], after $18 \mathrm{~h}$ the reduction was complete. The reaction mixture was diluted with ether (120 $\mathrm{ml})$ and treated with diethanolamine $(13.0 \mathrm{~g}, 124 \mathrm{mmol})$. After $2 \mathrm{~h}$, the separated solid was filtered off and the residue washed with pentane $(2 \times 80 \mathrm{ml})$. The combined filtrates were washed with saturated sodium bicarbonate $(100 \mathrm{ml})$, water $(2 \times 100 \mathrm{ml})$ and dried $\left(\mathrm{MgSO}_{4}\right)$. The solvents were distilled off (bath temperature $45^{\circ} \mathrm{C} / 15$ torr) and the remaining liquid (22.6 g) chromatographed on silica gel $(0.05-0.20 \mathrm{~mm})$ first in pentane, and, after all $\alpha$-pinene originating from the reagent had been eluted, in pentane/ether 8:2 [column 80 x $5.5 \mathrm{~cm} ; \mathrm{R}_{\mathrm{f}}=0.34(1 S, 4 S)-(+)-53,0.22$ $(1 S, 4 R)-(+)-51]$ to yield $2.27 \mathrm{~g}(21 \%)$ of $(1 S, 4 R)-(+)-51$ (purity 93\% GC) and $2.94 \mathrm{~g}(28 \%)$ of $(1 S, 4 S)-(+)-53$ (purity $96 \%$ GC) as colourless viscous liquids. The data given refer to chemically pure samples obtained by preparative GC. $(1 S, 4 R)-(+)-51:[\alpha]_{\mathrm{D}}{ }^{20}=+22.1$ (c 1.20, acetone); IR (neat): $3600-3100 \mathrm{~cm}^{-1}\left(\mathrm{OH}_{\mathrm{ass}}\right) ;{ }^{1} \mathrm{H}-\mathrm{NMR}\left(600 \mathrm{MHz}, \mathrm{CDCl}_{3}, \mathrm{CHCl}_{3}\right.$ int): $\delta=1.20-1.45(\mathrm{~m}, 8 \mathrm{H})$, 1.51-1.69 (m, 8H), 1.76-1.85 (br s, 1H), $2.01\left(\mathrm{~m}_{\mathrm{c}}, 1 \mathrm{H}\right), 2.08\left(\mathrm{~m}_{\mathrm{c}}, 1 \mathrm{H}\right), 3.99(\mathrm{dd}, \mathrm{J}=8,8 \mathrm{~Hz}, 1 \mathrm{H})$; ${ }^{13} \mathrm{C}-\mathrm{NMR}\left(150.8 \mathrm{MHz}, \mathrm{CDCl}_{3}, \mathrm{CDCl}_{3}\right.$ int): $\delta=19.69(\mathrm{t}), 21.36(\mathrm{t}), 24.46(\mathrm{t}), 24.58(\mathrm{t}), 27.86(\mathrm{t})$, $29.89(\mathrm{t}), 32.88(\mathrm{t}), 33.64(\mathrm{t}), 37.14(\mathrm{t}), 54.02(\mathrm{~s}), 58.06(\mathrm{~s}), 68.77$ (d); MS (EI): $m / e=180(<1$, $\mathrm{M}^{+}$), 121 (100). $\mathrm{C}_{12} \mathrm{H}_{20} \mathrm{O}$ requires $\mathrm{C}, 79.94 ; \mathrm{H}, 11.18$. Found: $\mathrm{C}, 79.67 ; \mathrm{H}, 10.97$. (1S,4S)-(+)-53: $[\alpha]_{\mathrm{D}}{ }^{20}=+1.21$ (c 1.24, acetone); IR (neat): $3620(\mathrm{OH}), 3600-3100 \mathrm{~cm}^{-1}\left(\mathrm{OH}_{\mathrm{ass}}\right) ;{ }^{1} \mathrm{H}-\mathrm{NMR}(600$ $\mathrm{MHz}, \mathrm{CDCl}_{3}, \mathrm{CHCl}_{3}$ int): $\delta=1.27-1.33(\mathrm{~m}, 1 \mathrm{H}), 1.41\left(\mathrm{~m}_{\mathrm{c}}, 1 \mathrm{H}\right), 1.45-1.69(\mathrm{~m}, 14 \mathrm{H}), 1.71-1.78$ $(\mathrm{m}, 1 \mathrm{H}), 1.85\left(\mathrm{~m}_{\mathrm{c}}, 1 \mathrm{H}\right), 2.23\left(\mathrm{~m}_{\mathrm{c}}, 1 \mathrm{H}\right), 4.11\left(\mathrm{~m}_{\mathrm{c}}, 1 \mathrm{H}\right) ;{ }^{13} \mathrm{C}-\mathrm{NMR}\left(150.8 \mathrm{MHz}, \mathrm{CDCl}_{3}, \mathrm{CDCl}_{3}\right.$ int $)$ : $\delta=19.29(\mathrm{t}), 22.60(\mathrm{t}), 24.11(\mathrm{t}), 25.70(\mathrm{t}), 28.21(\mathrm{t}), 33.18(\mathrm{t}), 34.70(\mathrm{t}), 37.86(\mathrm{t}), 39.36(\mathrm{t})$, 53.53 (s), 56.69 (s), $77.21(\mathrm{~d})$; MS (EI): m/e = $180\left(<1, \mathrm{M}^{+}\right), 95$ (100). HRMS m/e (M+) calcd 180.1514, obsd 180.1514. The optical purities of $(1 S, 4 R)-(+)-51(99 \%$ ee) and $(1 S, 4 S)-(+)-53$ (69\% ee) followed from the optical purities of the ketones (4R)-41 (99\% ee) and (4S)-41 (69\% ee) respectively, obtained by oxidation (see below).

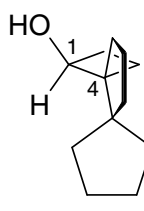

$(1 S, 4 R)-51$

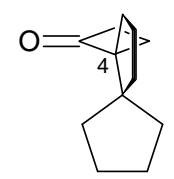

$(4 R)-41$ 
(4R)-(+)-Dispiro[3.0.4.3]dodecan-1-one $[(4 R)-(+)-41]$ : To a suspension of pyridinium chlorochromate $(5.12 \mathrm{~g}, 23.7 \mathrm{mmol})$ in dichloromethane $(12 \mathrm{ml})$ was added under argon with stirring a solution of $(1 S, 4 R)-(+)-51(2.27 \mathrm{~g}, 12.7 \mathrm{mmol}$, purity 93\%) in dichloromethane (12 ml) causing a slightly exothermic effect and the separation of a black grease. After $1 \mathrm{~h}$ at room temperature, TLC [pentane/ether 8:2, $\mathrm{R}_{\mathrm{f}}=0.50(\mathbf{4 1}), 0.22(\mathbf{5 1})$ ] indicated that the reaction was complete. The liquid phase was decanted and the residual grease extracted with ether (3 x $30 \mathrm{ml})$. The combined organic phases were filtrated through a short path of silica gel $(0.05-0.20 \mathrm{~mm}$, column $20 \times 4.5 \mathrm{~cm}$ ) and eluted with ether. The solvents were evaporated (bath temperature $40^{\circ} \mathrm{C} / 15$ torr $)$ and the residue (2.16 g yellow liquid) chromatographed on silica gel (0.05-0.20 $\mathrm{mm}$ ) in pentane/ether 95:5 (column $\left.65 \times 4.5 \mathrm{~cm} ; \mathrm{R}_{\mathrm{f}}=0.20[(4 R)-(+)-41]\right)$ to yield $1.30 \mathrm{~g}(61 \%)$ of $(4 R)-(+)-41$ as colourless liquid (purity 99\%, $>99 \%$ ee; $[\alpha]_{\mathrm{D}}{ }^{20}=+145, \mathrm{c}=1.23$, acetone). The chemical purity followed from a GC analysis [column A, $230^{\circ} \mathrm{C}$; retention time (min): $3.71(\mathbf{4 1})$ ]. The optical purity followed from the specific rotation of enantiopure material $\left([\alpha]_{\mathrm{D}}{ }^{20}=+145, \mathrm{c}=\right.$ 1.21 , acetone) obtained by thermolysis of $(\mathrm{S} S, 1 R, 4 R)-\mathbf{5 0}$ (see above). The ${ }^{1} \mathrm{H}$ and ${ }^{13} \mathrm{C}$ NMR data of both samples were identical.

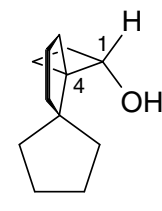

$(1 S, 4 S)-53$

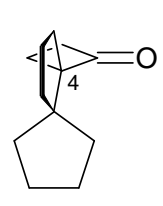

(4S)-41

(4S)-(-)-Dispiro[3.0.4.3] dodecan-1-one [(4S)-(-)-41]: The synthesis was performed as described for (4R)-(+)-41. $179 \mathrm{mg}(1.00 \mathrm{mmol})(1 S, 4 S)-(+)-53$ yielded $91 \mathrm{mg}(53 \%)$ of (4S)-(-)-41 (purity $99 \% \mathrm{GC}, 69 \% \mathrm{ee},[\alpha]_{\mathrm{D}}{ }^{20}=-84.4, \mathrm{c}=1.24$, acetone). The optical purity followed from the specific rotation of enantiopure material $\left([\alpha]_{\mathrm{D}}{ }^{20}=-145, \mathrm{c}=1.14\right.$, acetone $)$ obtained by thermolysis of $(\mathrm{S} S, 1 S, 4 S)-(+)-52$ (see above). The ${ }^{1} \mathrm{H}$ and ${ }^{13} \mathrm{C}$ NMR data of both samples were identical. 


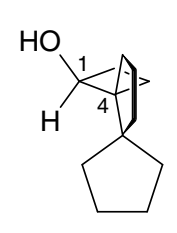

$(1 S, 4 R)-51$

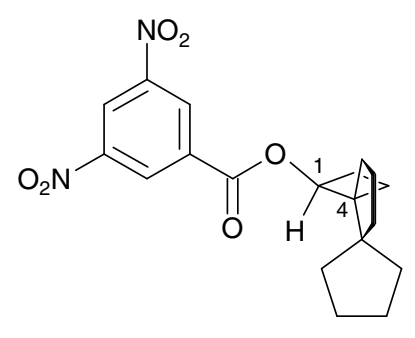

$(1 S, 4 R)-49$

$(1 S, 4 R)-(+)-3,5-D i n i t r o b e n z o i c$ acid dispiro[3.0.4.3]dodec-1-yl ester [(1S,4R)-(+)-49]: A solution of 3,5-dinitrobenzoic acid chloride (97 mg, $0.42 \mathrm{mmol})$ and (1S,4R)-(+)-51 (63 mg, 0.63 $\mathrm{mmol})$ in pyridine $(1.2 \mathrm{ml})$ was stirred for $21 \mathrm{~h}$ at room temperature until it was diluted with water $(3 \mathrm{ml})$ and extracted with dichloromethane. The extract was washed with water $(2 \times 3 \mathrm{ml})$, dried $\left(\mathrm{MgSO}_{4}\right)$ and concentrated (bath temperature $50^{\circ} \mathrm{C} / 15$ torr). The residue $(107 \mathrm{mg}$ ) was chromatographed on silica gel $(0.05-0.20 \mathrm{~mm})$ in pentane/ether $8: 2$ [column $10 \mathrm{x} 1 \mathrm{~cm}, \mathrm{R}_{\mathrm{f}}=0.48$ (49)] yielding $97 \mathrm{mg}$ of $(1 S, 4 R)-(+)-49$ as colourless solid. Crystallization from acetone $(2.0 \mathrm{ml})$ by diffusion of water $(2.5 \mathrm{ml})$ gave $87 \mathrm{mg}(66 \%)$ of pure $(1 S, 4 R)-(+)-49$ as colourless crystals, $\mathrm{mp} 81-84^{\circ} \mathrm{C},[\alpha]_{\mathrm{D}}{ }^{20}=+95.0(\mathrm{c}=1.19$, acetone $)$. IR $(\mathrm{KBr}): 1725 \mathrm{~cm}^{-1}(\mathrm{C}=\mathrm{O}) ;{ }^{1} \mathrm{H}-\mathrm{NMR}(600 \mathrm{MHz}$, $\mathrm{C}_{6} \mathrm{D}_{6}, \mathrm{C}_{6} \mathrm{D}_{5} \mathrm{H}$ int): $\delta=1.12-1.18(\mathrm{~m}, 1 \mathrm{H}), 1.19-1.25(\mathrm{~m}, 1 \mathrm{H}), 1.29-1.45(\mathrm{~m}, 6 \mathrm{H}), 1.53-1.66(\mathrm{~m}$, $7 \mathrm{H}), 1.90\left(\mathrm{~m}_{\mathrm{c}}, 1 \mathrm{H}\right), 2.22\left(\mathrm{~m}_{\mathrm{c}}, 1 \mathrm{H}\right), 2.27-2.31(\mathrm{~m}, 1 \mathrm{H}), 5.27(\mathrm{dd}, \mathrm{J}=8,8 \mathrm{~Hz}, 1 \mathrm{H}), 8.49(\mathrm{t}, \mathrm{J}=2$ $\mathrm{Hz}, 1 \mathrm{H}), 8.72$ (d, J = $2 \mathrm{~Hz}, 2 \mathrm{H}):{ }^{13} \mathrm{C}-\mathrm{NMR}\left(150.8 \mathrm{MHz}, \mathrm{C}_{6} \mathrm{D}_{6}, \mathrm{C}_{6} \mathrm{D}_{6} \mathrm{int}\right): \delta=20.27$ (t), 21.87 (t), $24.82(\mathrm{t}), 25.02(\mathrm{t}), 25.48(\mathrm{t}), 31.83(\mathrm{t}), 33.34(\mathrm{t}), 33.60(\mathrm{t}), 36.98(\mathrm{t}), 54.26(\mathrm{~s}), 57.47(\mathrm{~s}), 72.54$ (d), 121.90 (d), 128.52 (d), 133.44 (s), 148.29 (s), 161.53 (s); MS (EI): $m / e=374\left(<1, \mathrm{M}^{+}\right), 121$ (100). $\mathrm{C}_{19} \mathrm{H}_{22} \mathrm{~N}_{2} \mathrm{O}_{6}$ requires $\mathrm{C}, 60.95 ; \mathrm{H}, 5.92$. Found: C, 61.00; H, 5.98.

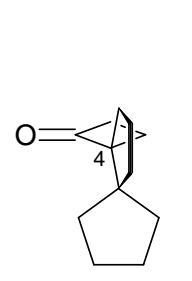

$(4 R)-41$

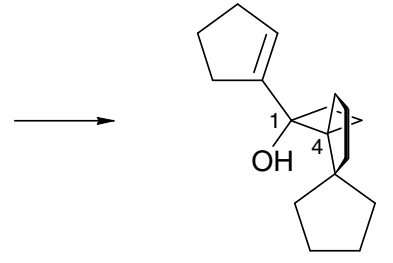

$(1 S, 4 R)-42$

$(1 S, 4 R)-(-)-1-C y c l o p e n t-1-e n y l-d i s p i r o[3.0 .4 .3]$ dodecan-1-ol [(1S,4R)-(-)-42]: The synthesis was performed as described for racemic 42. $2.19 \mathrm{~g}$ (12.3 mmol) (4R)-(+)-41 (purity 99\%, >99\% ee) yielded $2.78 \mathrm{~g}(88 \%)(1 S, 4 R)-(-)-42$ (purity 95\%, $>99 \%$ ee, $[\alpha]_{\mathrm{D}}{ }^{20}=-46.1, \mathrm{c}=1.30$, acetone). The ${ }^{1} \mathrm{H}$ - und ${ }^{13} \mathrm{C}$ NMR data were identical with those of racemic $\mathbf{4 2}$. 


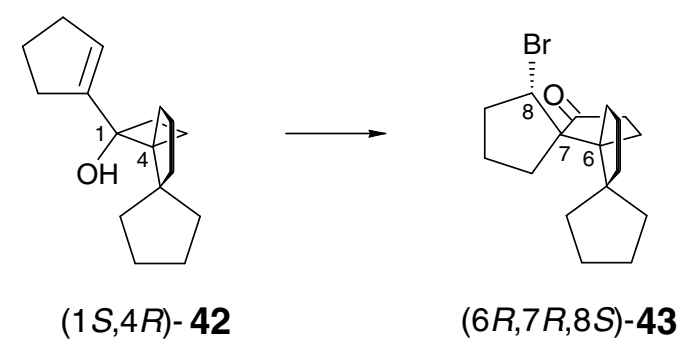

$(6 R, 7 R, 8 S)-(-)-8$-Brom-trispiro[4.0.0.4.3.3] heptadecan-12-one $\quad[(6 R, 7 R, 8 S)-(-)-43]: \quad$ The synthesis was performed as described for racemic 43. $2.71 \mathrm{~g}(11.0 \mathrm{mmol})(1 S, 4 R)-(-)-42$ (purity $95 \%,>99 \%$ ee) yielded $2.55 \mathrm{~g}(75 \%)(6 R, 7 R, 8 S)-(-)-43$ (purity 99\%, >99\% ee, $[\alpha]_{\mathrm{D}}{ }^{20}=-46.3, \mathrm{c}$ $=1.24$, acetone). The ${ }^{1} \mathrm{H}$ and ${ }^{13} \mathrm{C}$ NMR data were identical with those of racemic 43 .

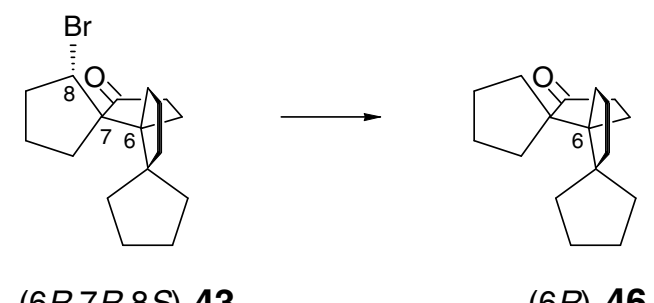

$(6 R, 7 R, 8 S)-43$

$(6 R)-46$

(6R)-(-)-Trispiro[4.0.0.4.3.3]heptadecan-12-one [(6R)-(-)-46]: The synthesis was performed as described for racemic 46. $2.47 \mathrm{~g}(7.58 \mathrm{mmol})(6 R, 7 R, 8 S)-(-)-43$ (purity 99\%, >99\% ee) yielded $1.37 \mathrm{~g}(73 \%)(6 R)-(-)-46$ (purity $99 \%,>99 \%$ ee, $[\alpha]_{\mathrm{D}}{ }^{20}=-127.0, \mathrm{c}=1.21$, acetone). The ${ }^{1} \mathrm{H}$ and ${ }^{13} \mathrm{C}$ NMR data were identical with those of racemic $\mathbf{4 6}$.

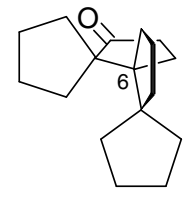

$(6 R)-46$

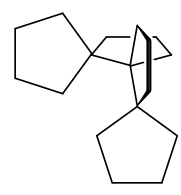

$(P)-9$

$(\boldsymbol{P})-(-)$-Trispiro[4.0.0.4.3.3]heptadecane $[(\boldsymbol{P})-(-)-9]:$ The synthesis was performed as described for racemic 9. $961 \mathrm{mg}$ (3.90 mmol) (6R)-(-)-46 (purity 99\%, >99\% ee) yielded $729 \mathrm{mg}(80 \%)$ $(P)-(-)-9$ (purity 98\%, >99\% ee, $\left.[\alpha]_{\mathrm{D}}{ }^{20}=-62.6, \mathrm{c}=1.10, \mathrm{CHCl}_{3}\right)$. The ${ }^{1} \mathrm{H}$ and ${ }^{13} \mathrm{C} \mathrm{NMR}$ data were identical with those of racemic 9. 


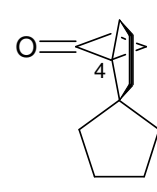

(4R)-41

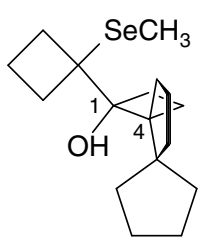

$(1 R, 4 R)-48$

$(1 R, 4 R)-1-\left[\left(1^{\prime}\right.\right.$-Methylselanyl)cyclobutyl]dispiro[3.0.4.3]dodecan-1-ol $\quad[(1 R, 4 R)-48]: \quad$ The synthesis was performed as described for racemic 48. $1.50 \mathrm{~g}$ (8.43 mmol) (4R)-(+)-41 (purity $99 \%,>99 \%$ ee) yielded $1.56 \mathrm{~g}(40 \%),(1 R, 4 R)-48$ as $70: 30$ mixture with $(4 R)-(+)-41$. The ${ }^{1} \mathrm{H}$ und ${ }^{13} \mathrm{C}$ NMR data were identical with those of a mixture of racemic $\mathbf{4 8}$ and $\mathbf{4 1}$.

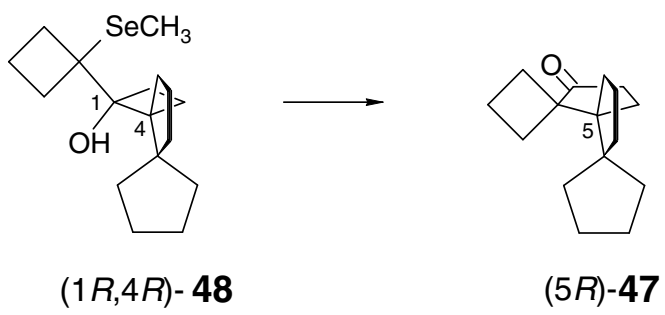

(5R)-(-)-Trispiro[3.0.0.4.3.3]hexadecan-16-one [(5R)-(-)-47]: The synthesis was performed as described for racemic 47. $1.55 \mathrm{~g}(4.74 \mathrm{mmol})(1 R, 4 R)-48$ (purity 70\%) yielded $595 \mathrm{mg}(76 \%)$ $(5 R)-(-)-47$ (purity 99\%, >99\% ee, $[\alpha]_{\mathrm{D}}{ }^{20}=-12.7, \mathrm{c}=1.23$, acetone). The ${ }^{1} \mathrm{H}$ and ${ }^{13} \mathrm{C}$ NMR data were identical with those of racemic 47.

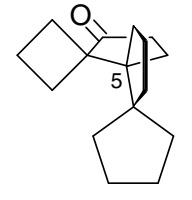

(5R)-47

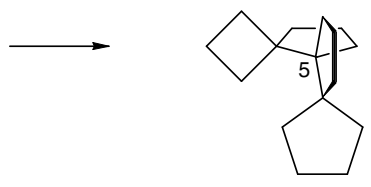

$(5 R)-8$

(5R)-(-)-Trispiro[3.0.0.4.3.3]hexadecane [(5R)-(-)-8]: The synthesis was performed as described for racemic 8. $464 \mathrm{mg}(2.00 \mathrm{mmol})(5 R)-(-)-47$ (purity 99\%, >99\% ee) yielded $287 \mathrm{mg}$ $(66 \%)(5 R)-(-)-8$ (purity 98\%, $>99 \%$ ee, $\left.[\alpha]_{\mathrm{D}}{ }^{20}=-30.7, \mathrm{c}=1.15, \mathrm{CHCl}_{3}\right)$. The ${ }^{1} \mathrm{H}$ and ${ }^{13} \mathrm{C} \mathrm{NMR}$ data were identical with those of racemic $\mathbf{8}$. 


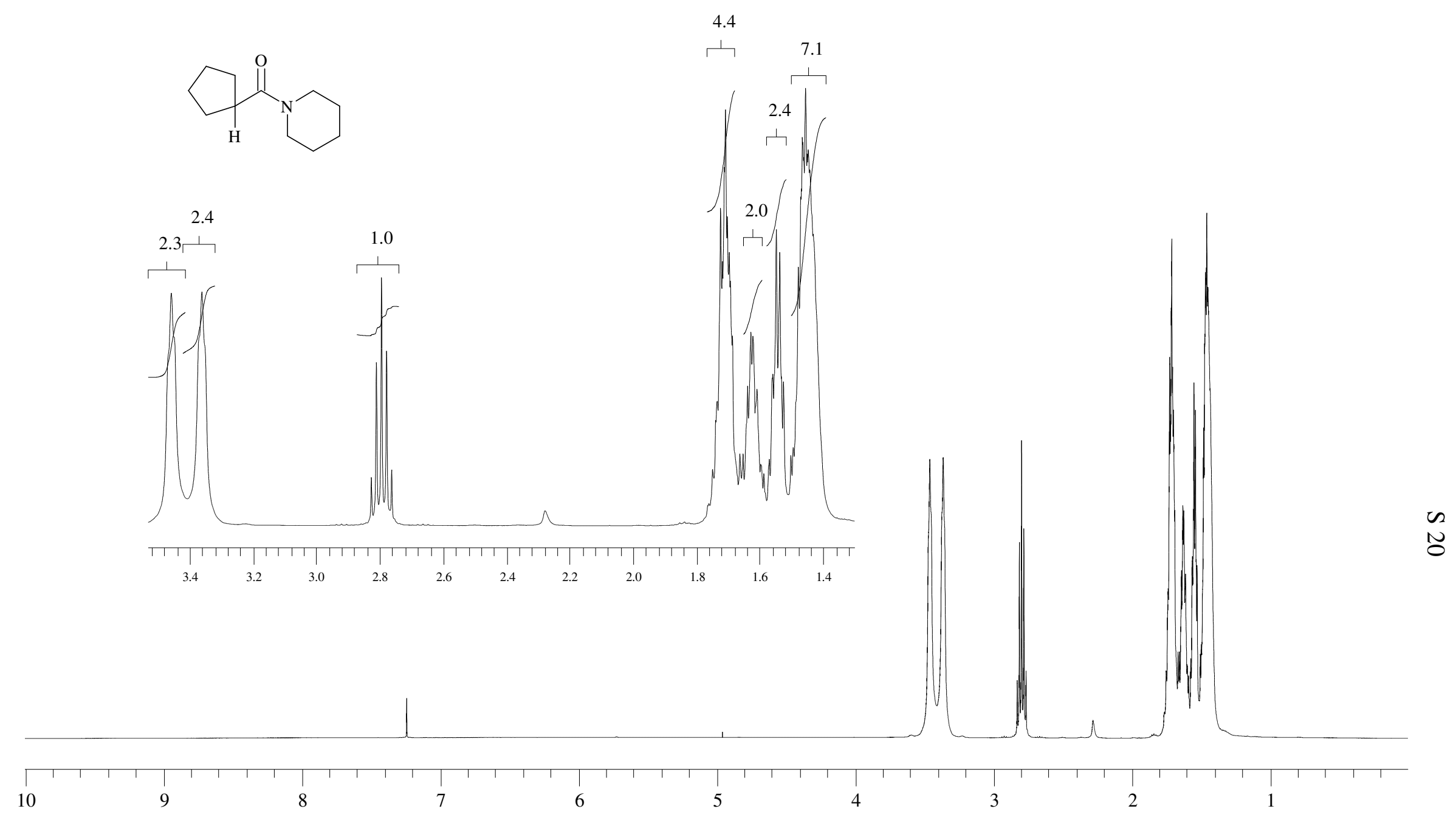

${ }^{1} \mathrm{H}$-NMR spectrum $\left(500 \mathrm{MHz}, \mathrm{CDCl}_{3}\right.$ ) of $\mathbf{2 0}$ 

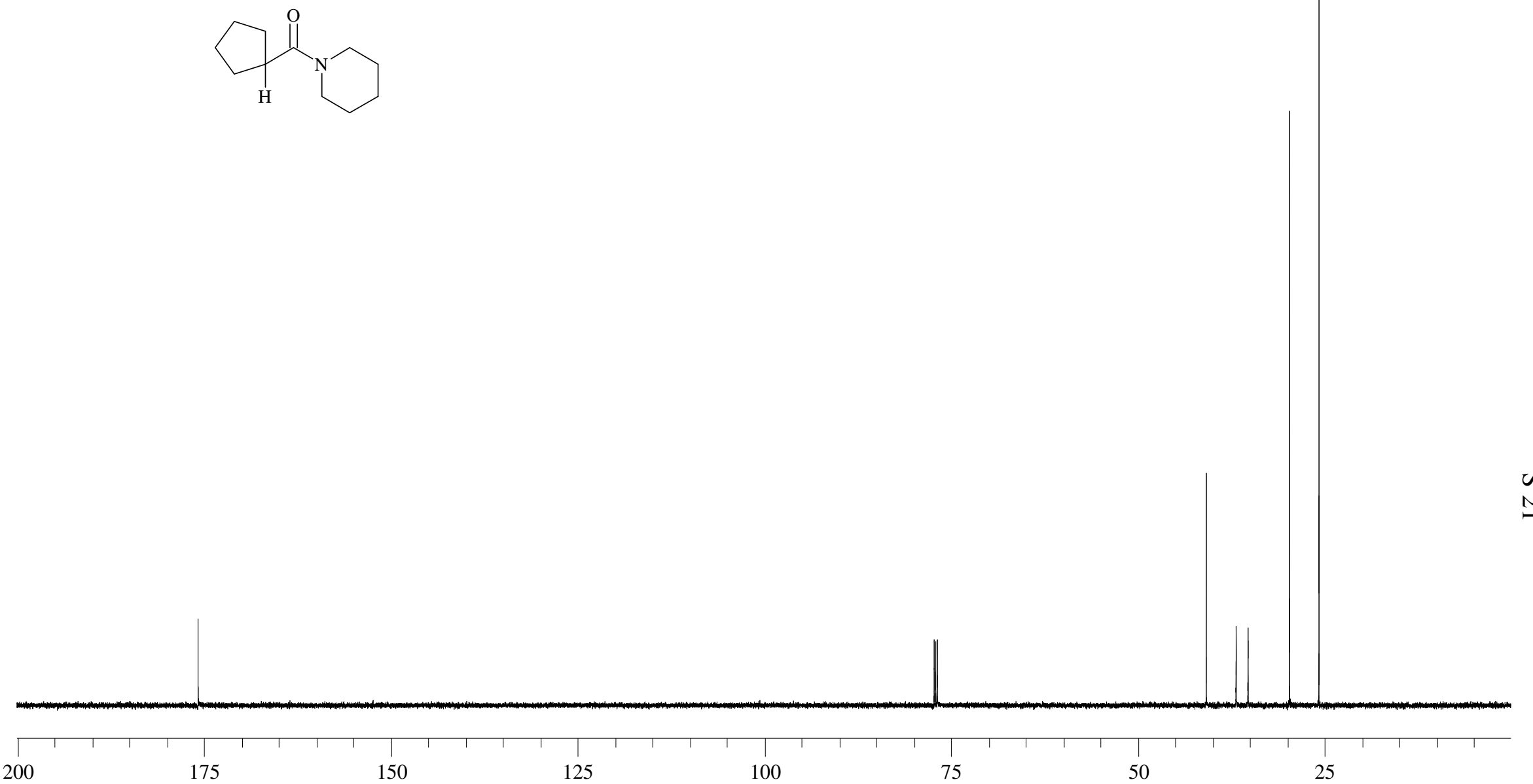

${ }^{13} \mathrm{C}-\mathrm{NMR}$ spectrum $\left(150.8 \mathrm{MHz}, \mathrm{CDCl}_{3}\right)$ of $\mathbf{2 0}$ 

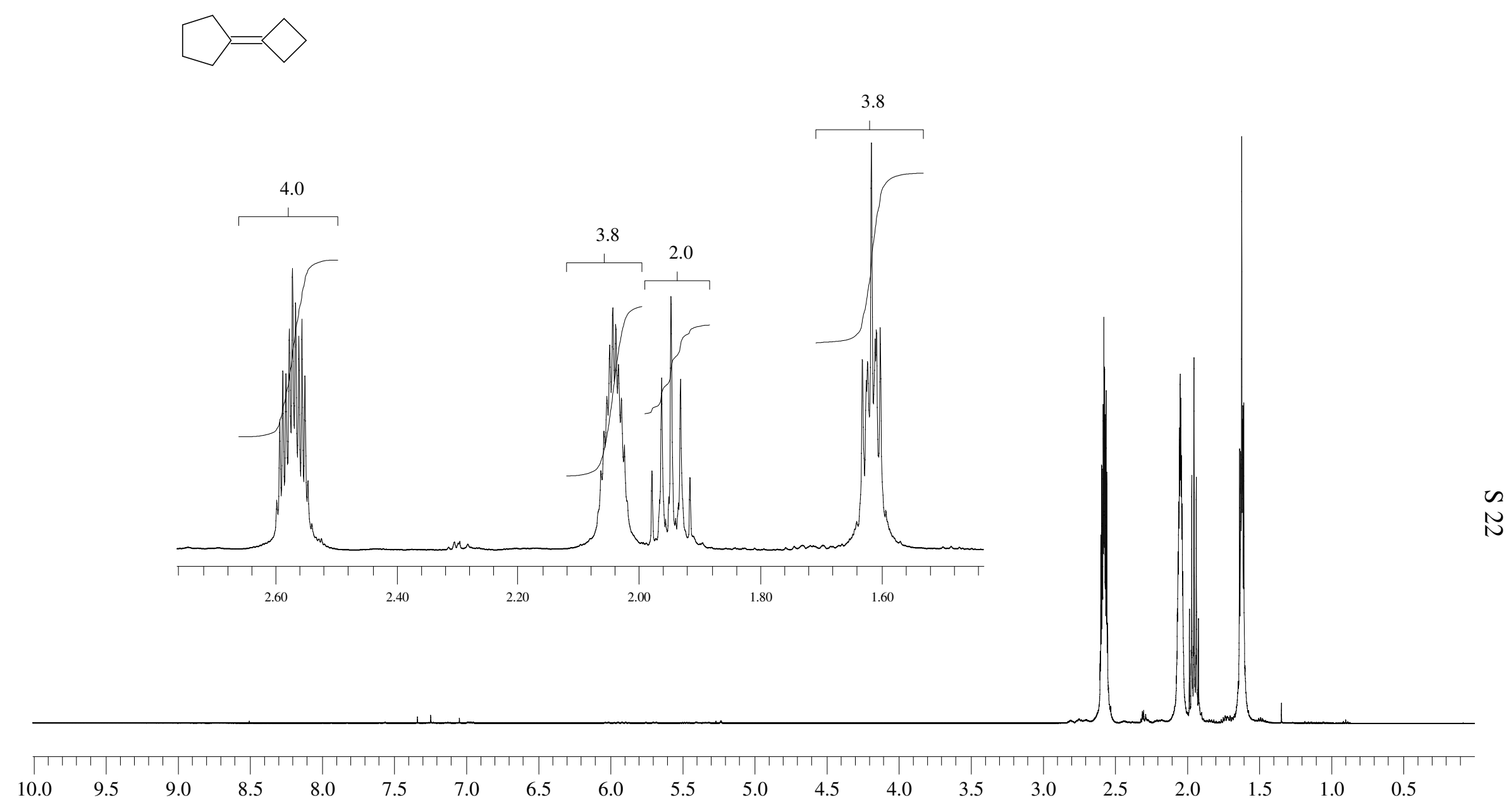

U

${ }^{1} \mathrm{H}-\mathrm{NMR}$ spectrum $\left(500 \mathrm{MHz}, \mathrm{CDCl}_{3}\right.$ ) of 22 

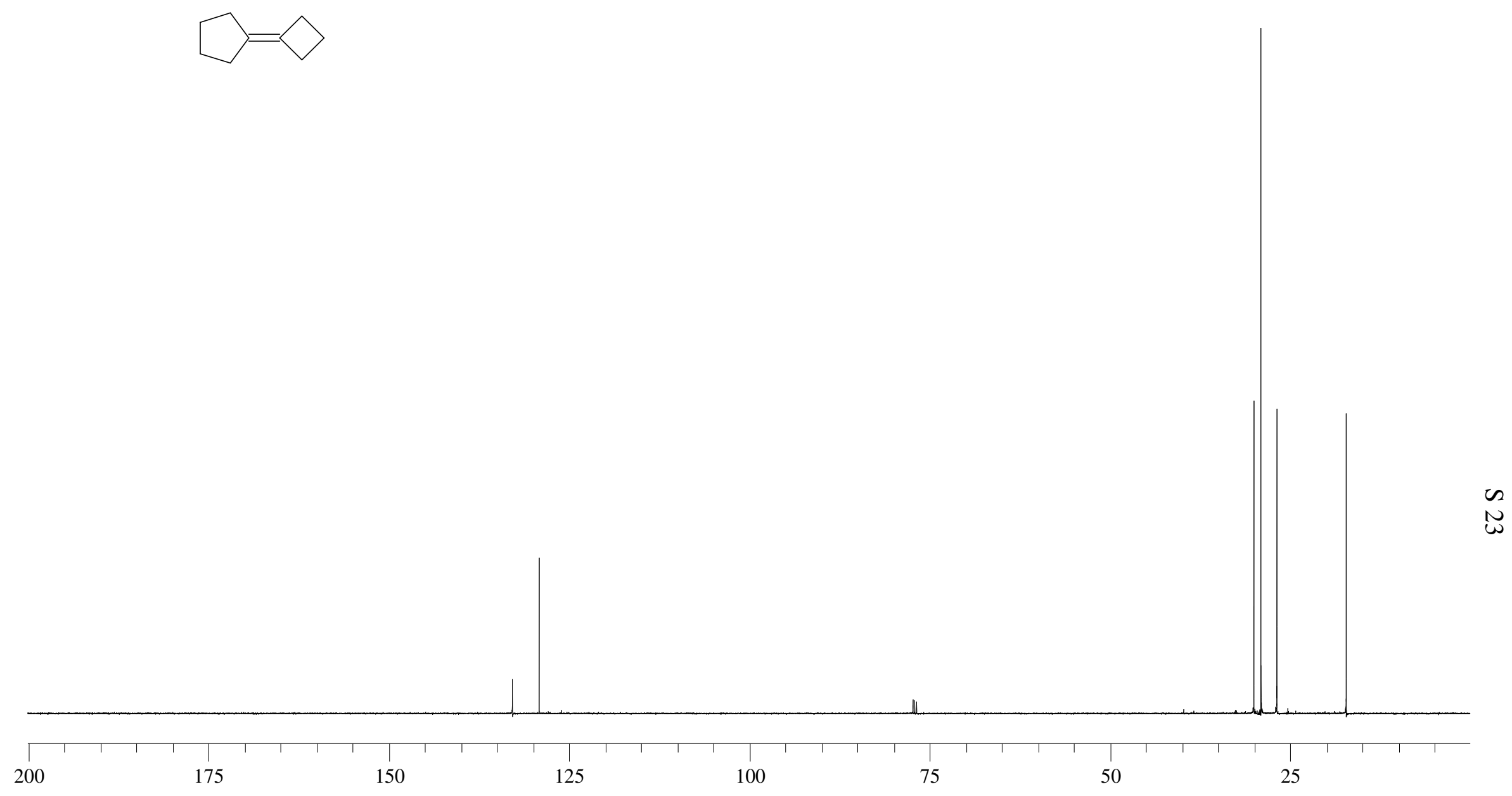

${ }^{13} \mathrm{C}-\mathrm{NMR}$ spectrum $\left(125.8 \mathrm{MHz}, \mathrm{CDCl}_{3}\right)$ of $\mathbf{2 2}$ 

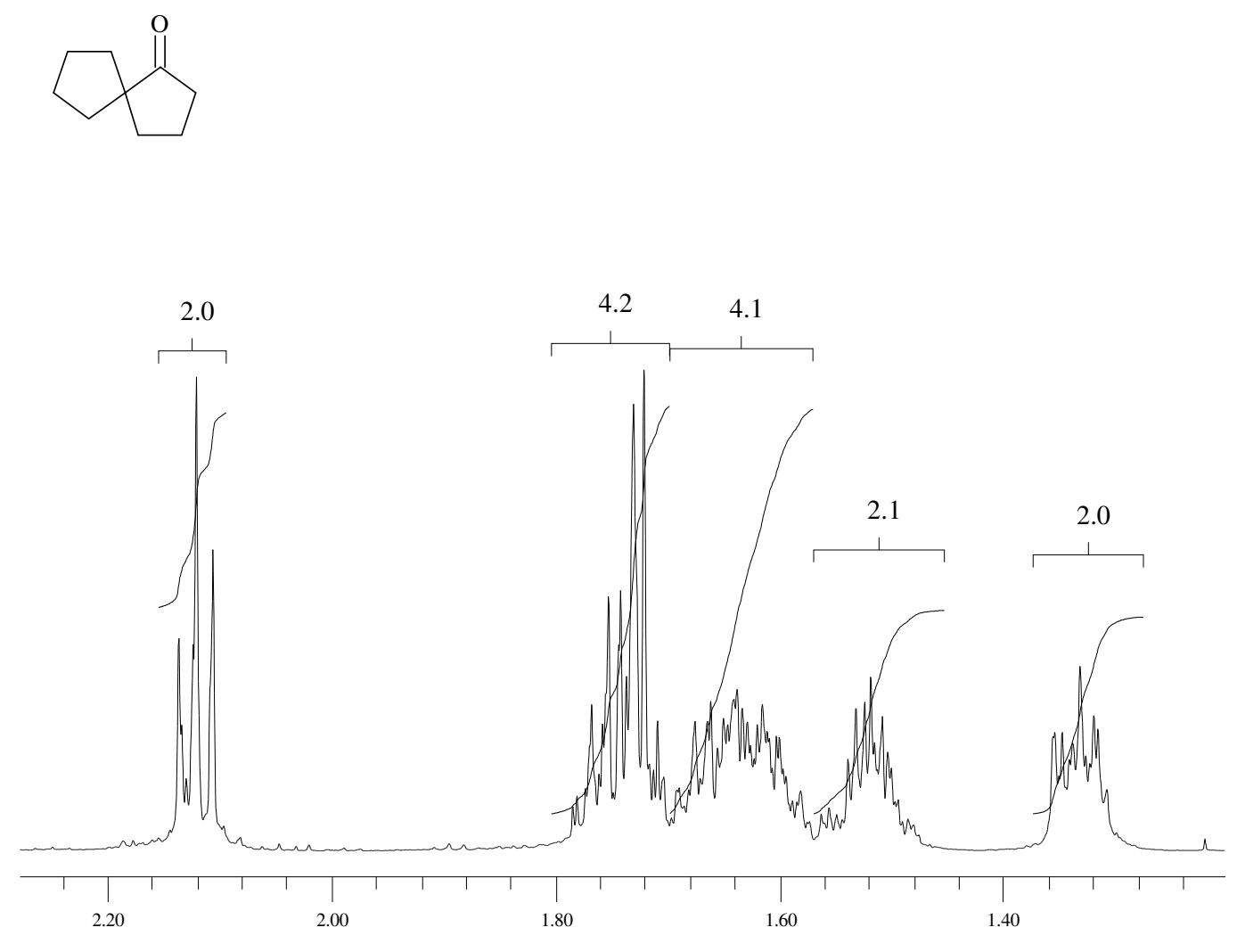

${ }^{1} \mathrm{H}-\mathrm{NMR}$ spectrum $\left(500 \mathrm{MHz}, \mathrm{CDCl}_{3}\right.$ ) of $\mathbf{2 3}$ 


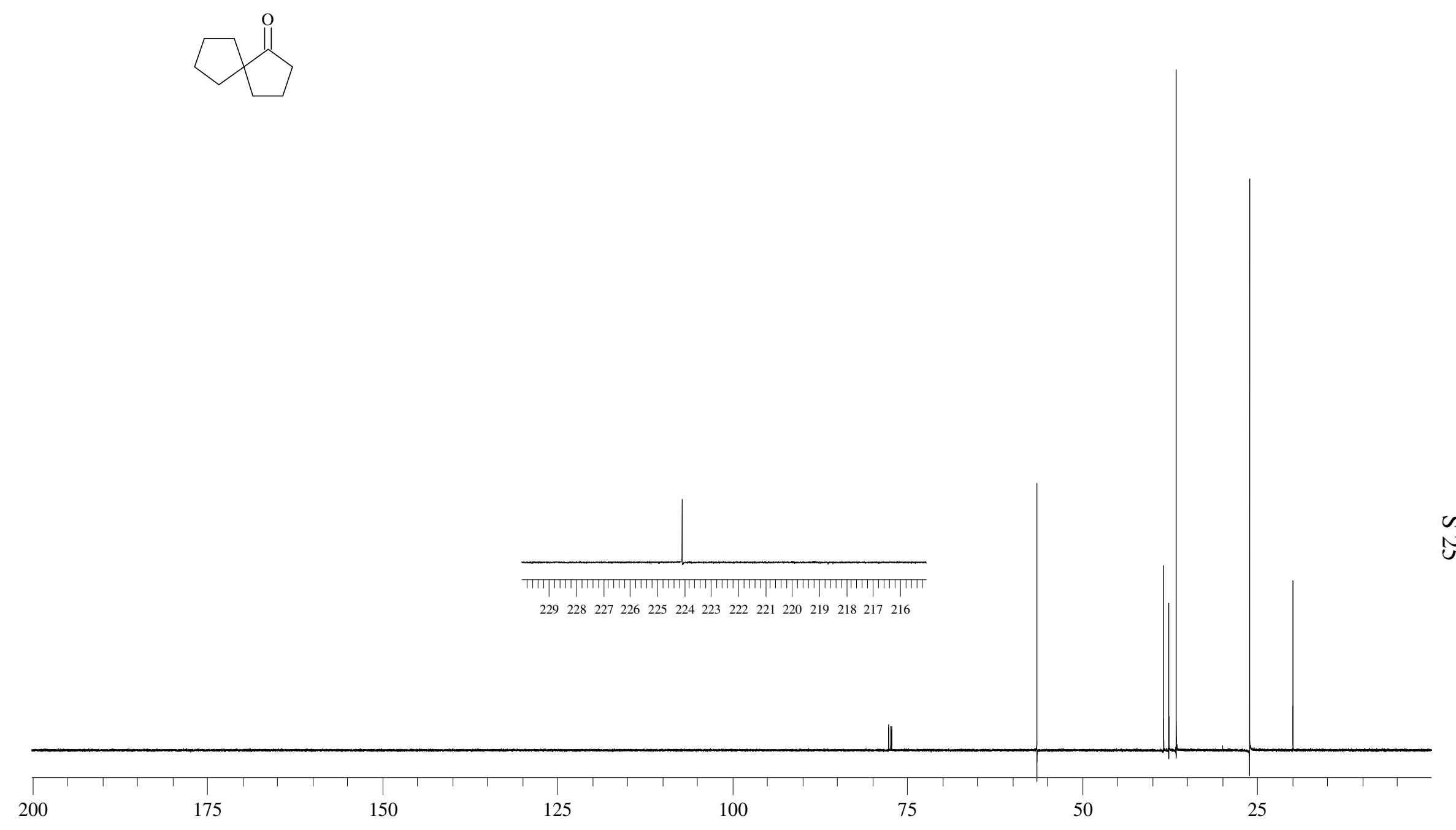

${ }^{13} \mathrm{C}$-NMR spectrum $\left(125.8 \mathrm{MHz}, \mathrm{CDCl}_{3}\right)$ of $\mathbf{2 3}$ 


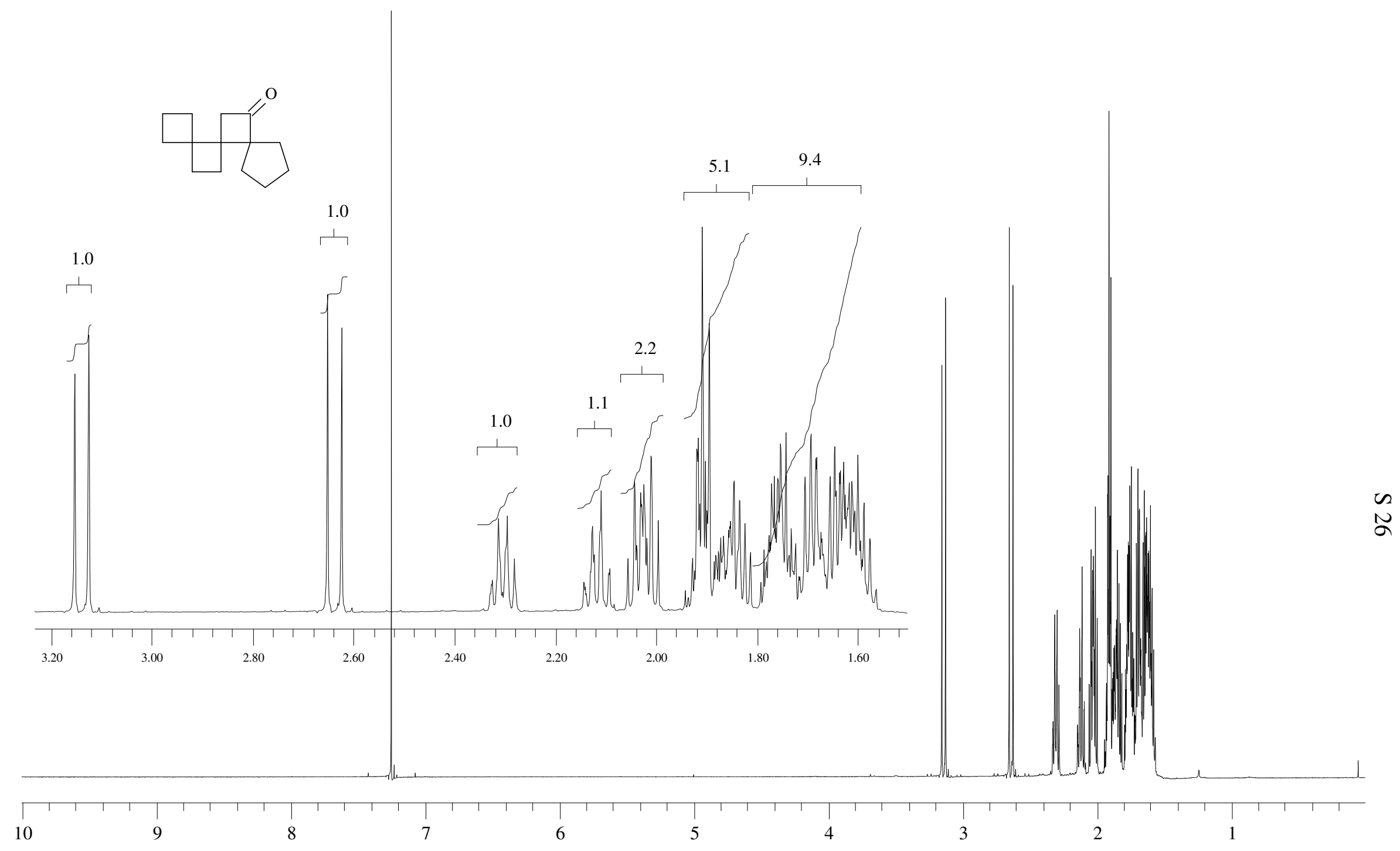

${ }^{1} \mathrm{H}$-NMR spectrum $\left(600 \mathrm{MHz}, \mathrm{CDCl}_{3}\right.$ ) of $\mathbf{1 5}$ 

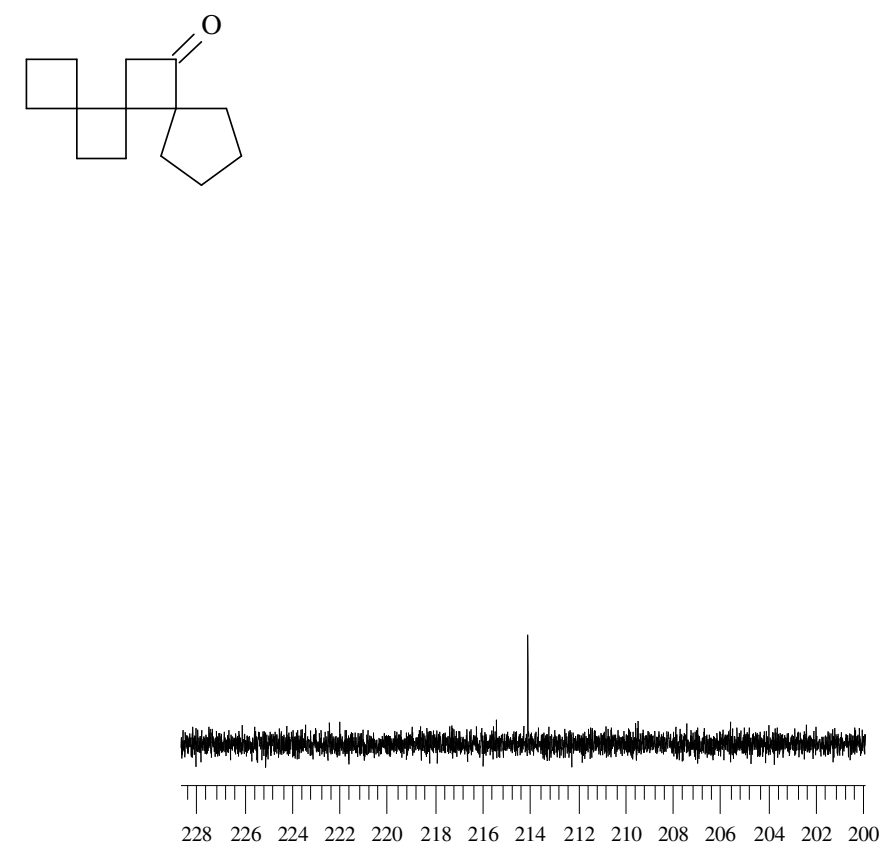

$\begin{array}{lllllllllllllll}228 & 226 & 224 & 222 & 220 & 218 & 216 & 214 & 212 & 210 & 208 & 206 & 204 & 202 & 200\end{array}$

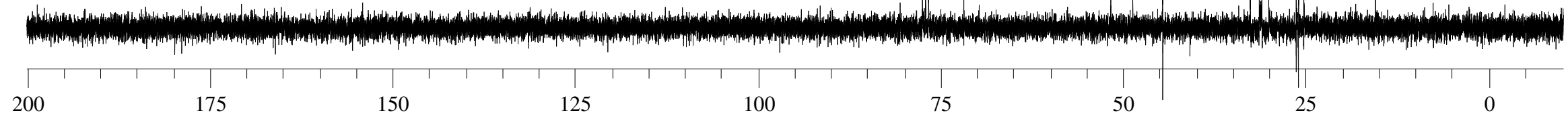

${ }^{13} \mathrm{C}$-NMR spectrum (75.5 MHz, $\mathrm{CDCl}_{3}$ ) of $\mathbf{1 5}$ 


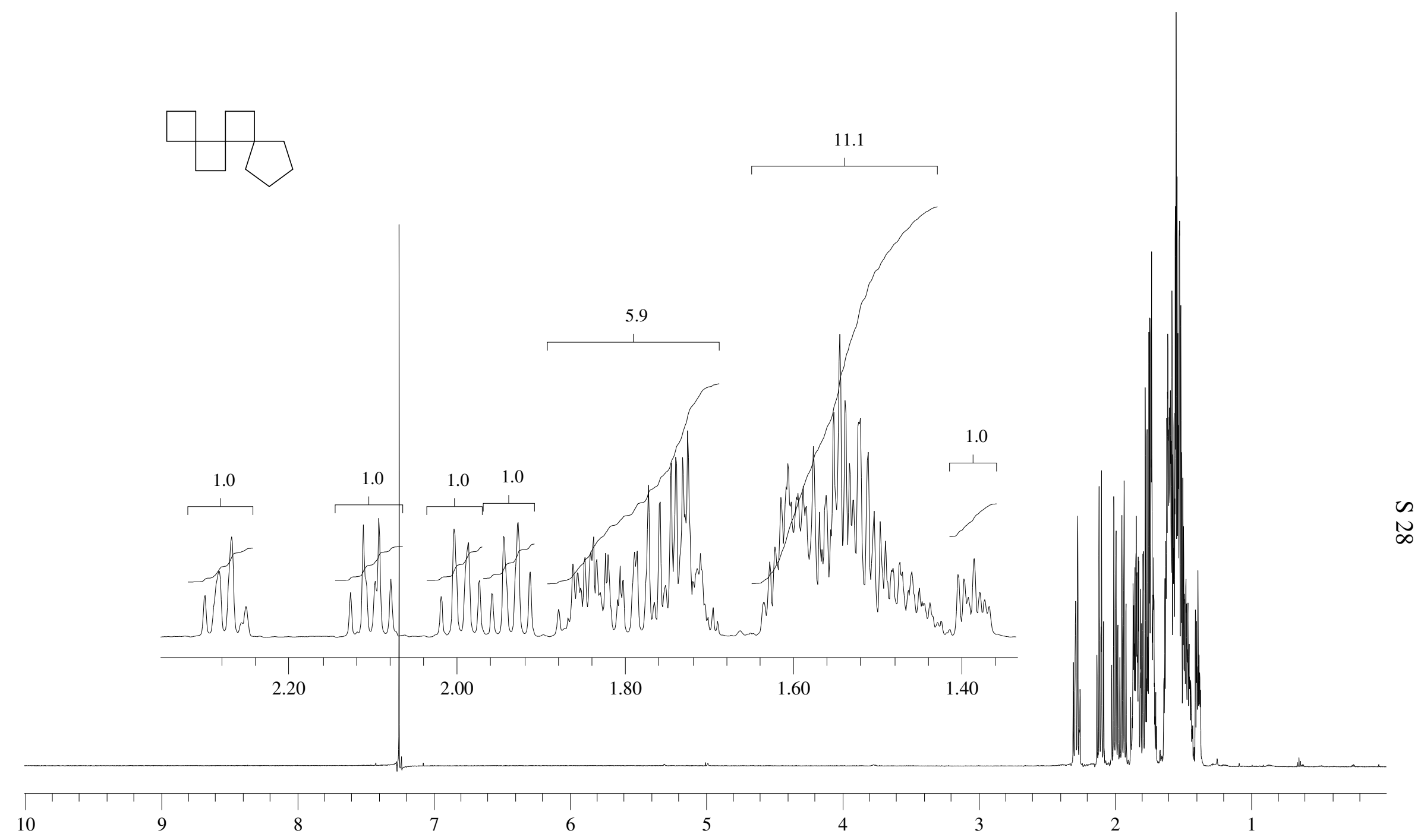

${ }^{1} \mathrm{H}-\mathrm{NMR}$ spectrum $\left(600 \mathrm{MHz}, \mathrm{CDCl}_{3}\right)$ of $\mathbf{6}$ 

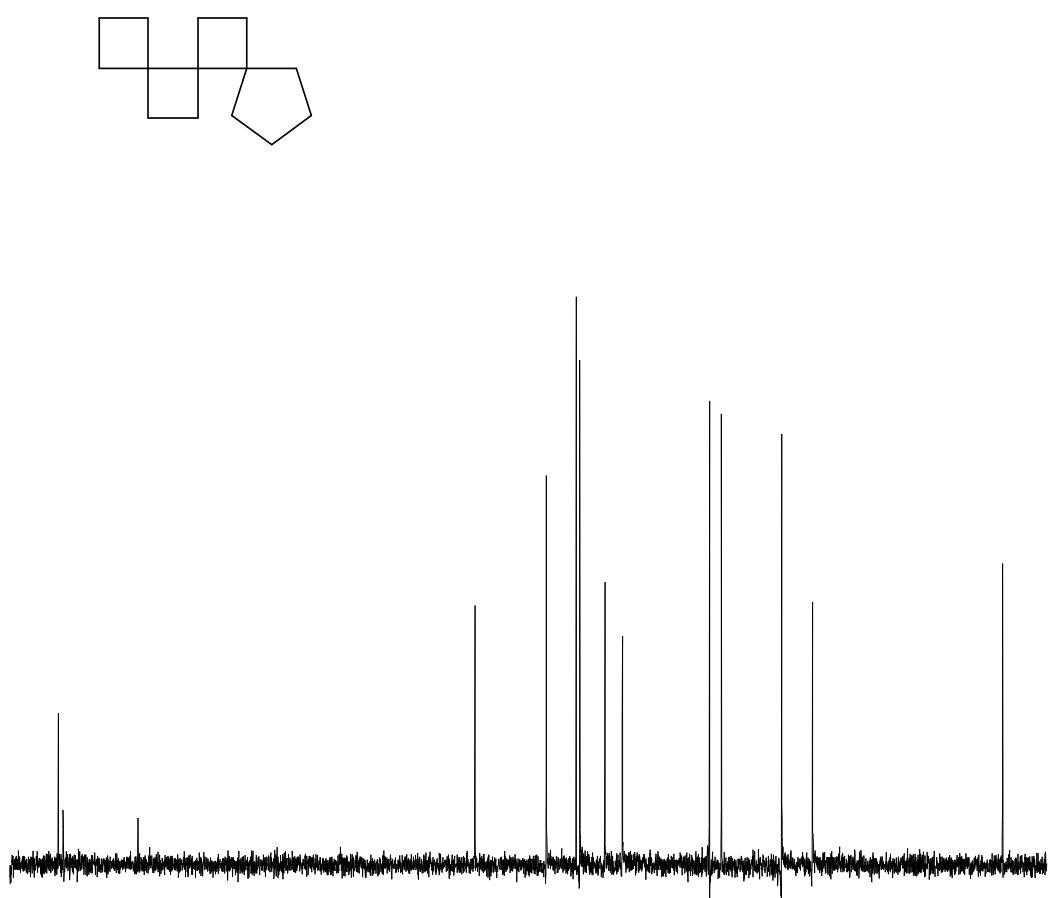

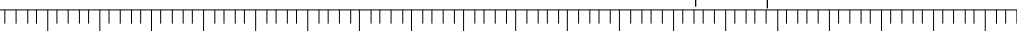
$\begin{array}{lllllllllllllllllll}52 & 50 & 48 & 46 & 44 & 42 & 40 & 38 & 36 & 34 & 32 & 30 & 28 & 26 & 24 & 22 & 20 & 18 & 16\end{array}$

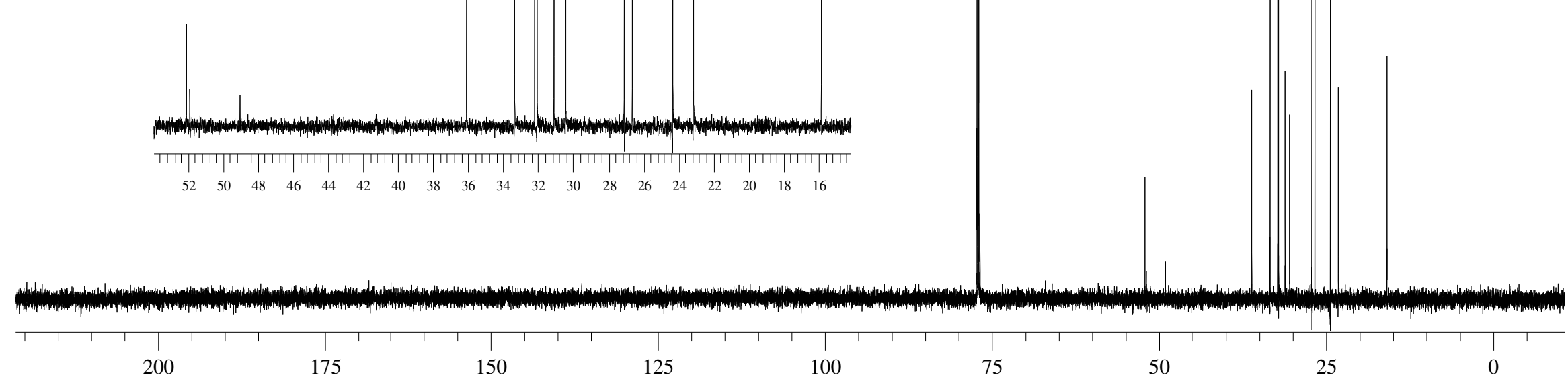

${ }^{13} \mathrm{C}-\mathrm{NMR}$ spectrum $\left(75.5 \mathrm{MHz}, \mathrm{CDCl}_{3}\right)$ of 6 


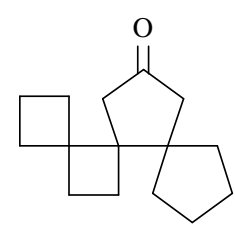

4.4

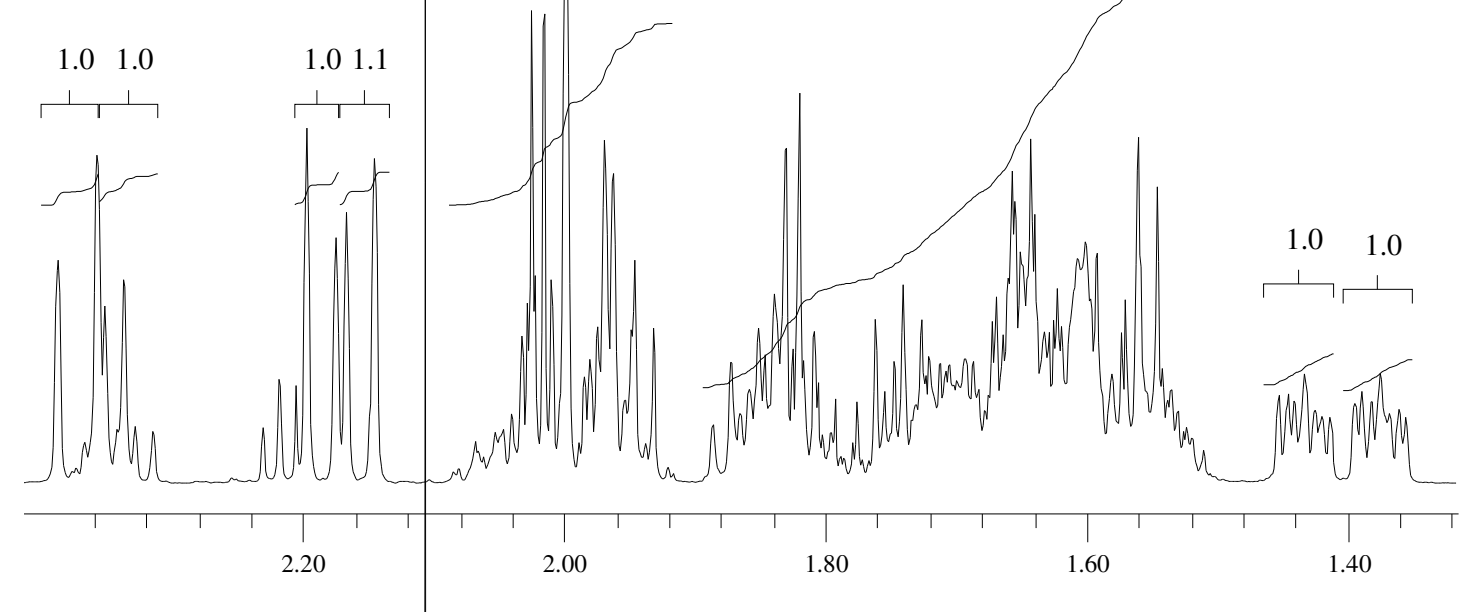

$\ddot{\circ}$

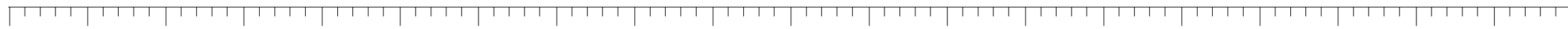

\section{${ }^{1} \mathrm{H}-\mathrm{NMR}$ spectrum $\left(600 \mathrm{MHz}, \mathrm{CDCl}_{3}\right)$ of $\mathbf{2 4}$}



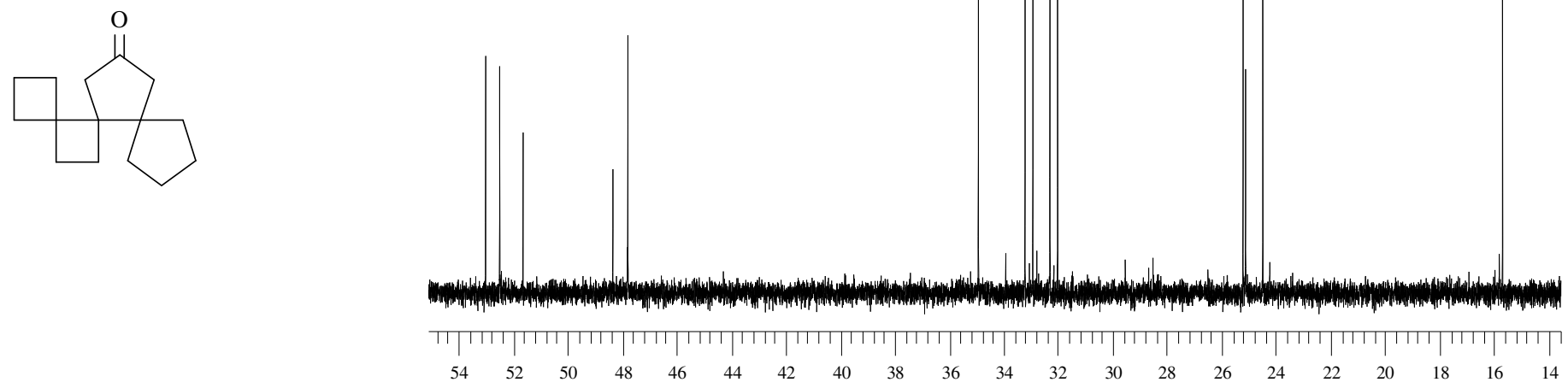

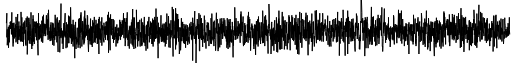

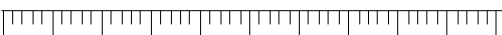

$\begin{array}{lllllllllll}226 & 225 & 224 & 223 & 222 & 221 & 220 & 219 & 218 & 217 & 216\end{array}$

-NMR spectrum $\left(150.8 \mathrm{MHz}, \mathrm{CDCl}_{3}\right)$ of $\mathbf{2 4}$ 


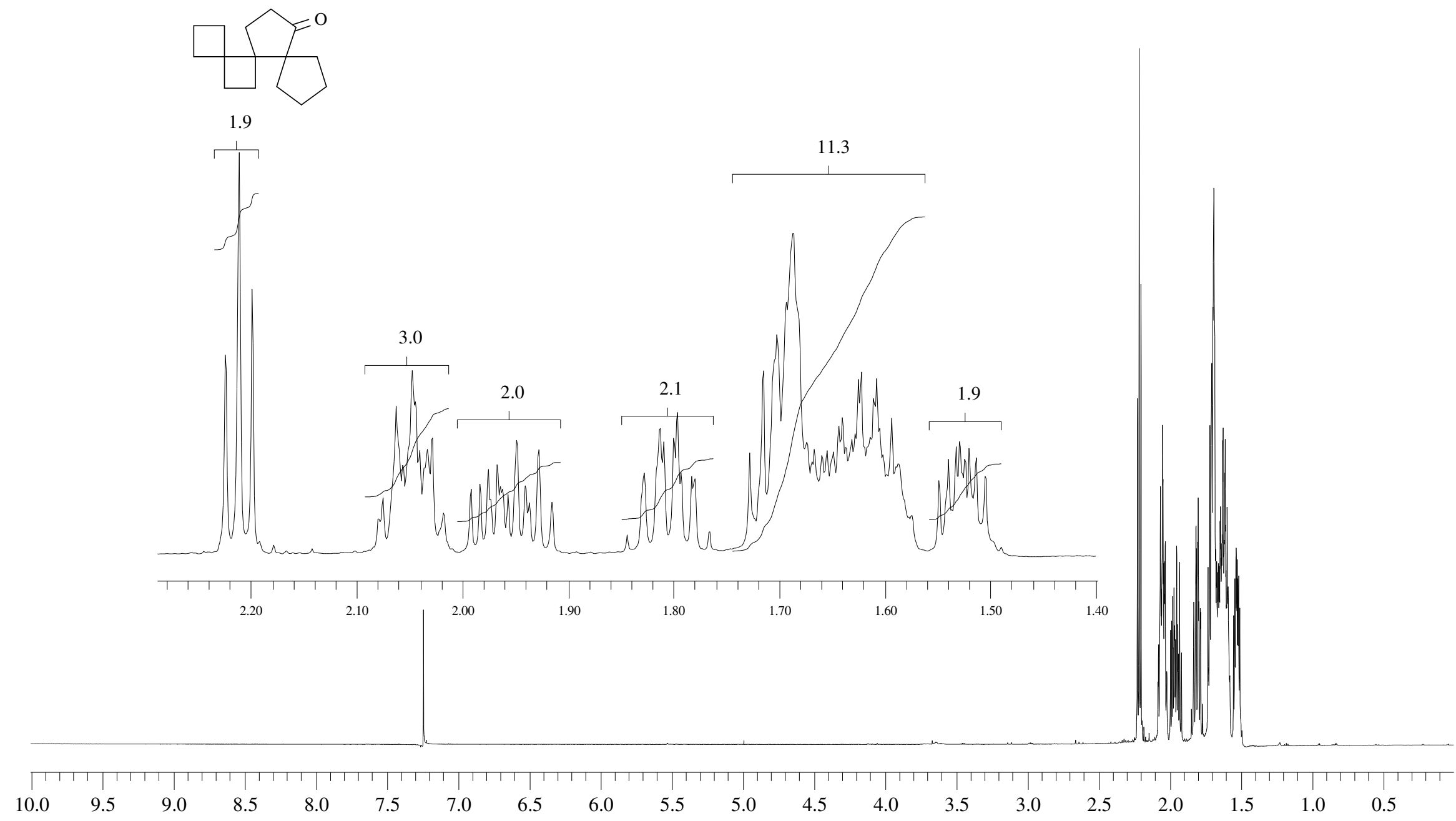

${ }^{1} \mathrm{H}$-NMR spectrum $\left(600 \mathrm{MHz}, \mathrm{CDCl}_{3}\right.$ ) of $\mathbf{2 5}$ 

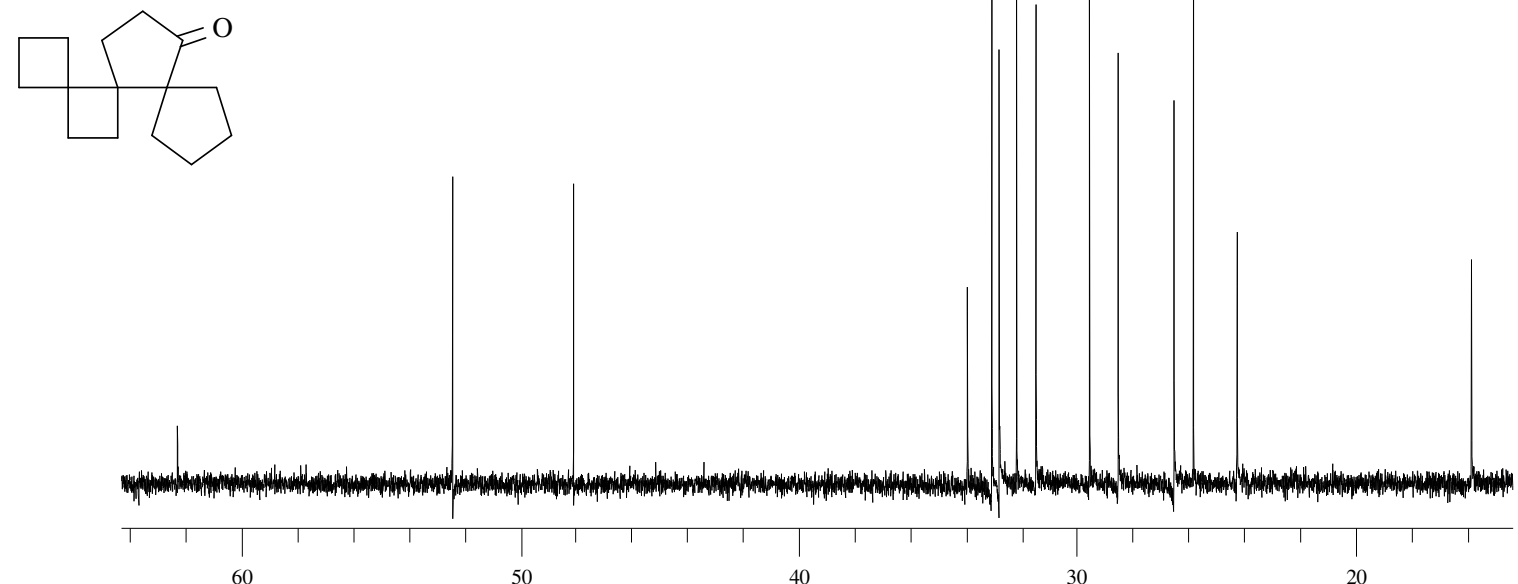

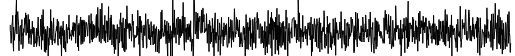

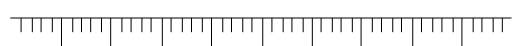

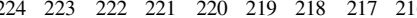

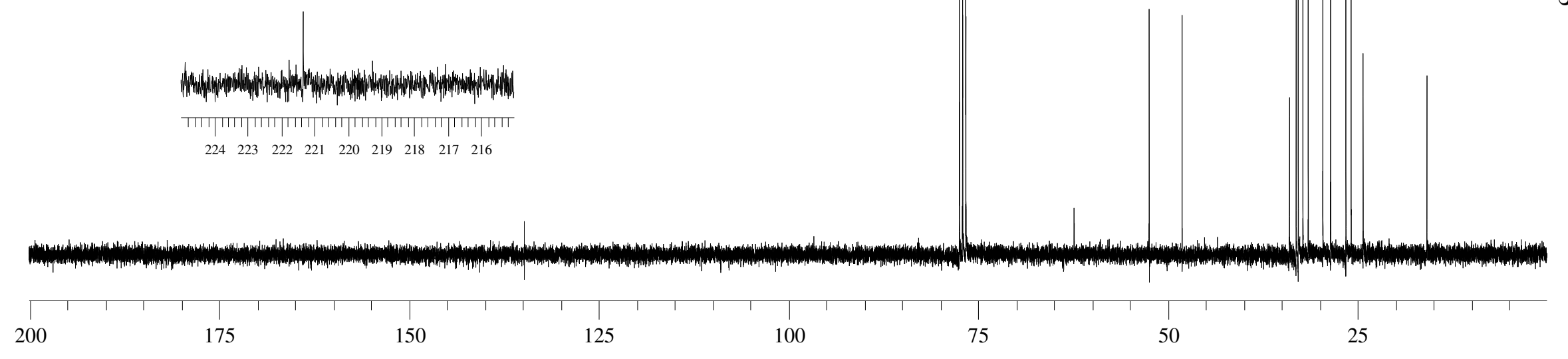

${ }^{13} \mathrm{C}$-NMR spectrum (75.5 MHz, $\mathrm{CDCl}_{3}$ ) of $\mathbf{2 5}$ 


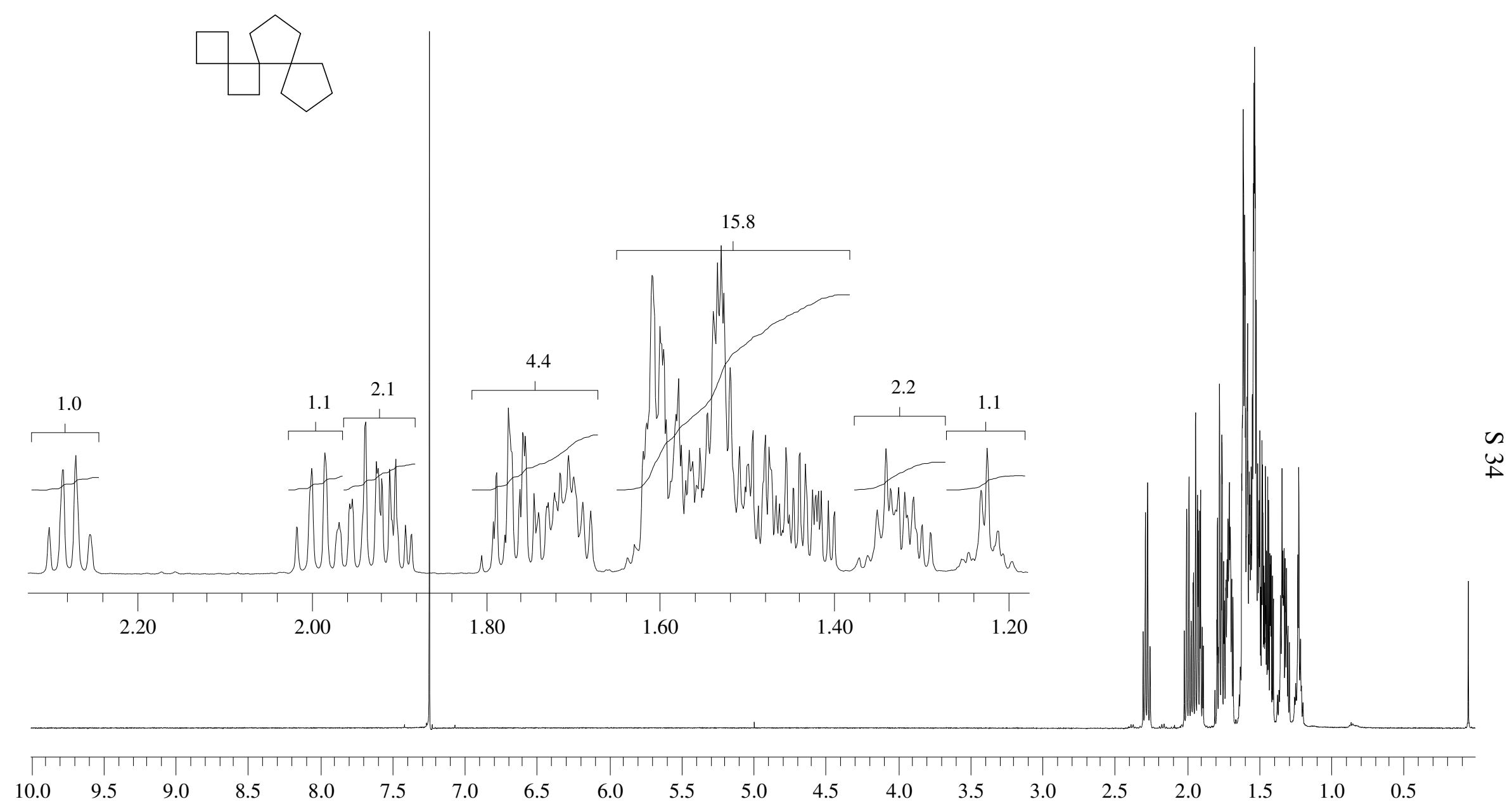

${ }^{1} \mathrm{H}-\mathrm{NMR}$ spectrum $\left(600 \mathrm{MHz}, \mathrm{CDCl}_{3}\right)$ of 7 


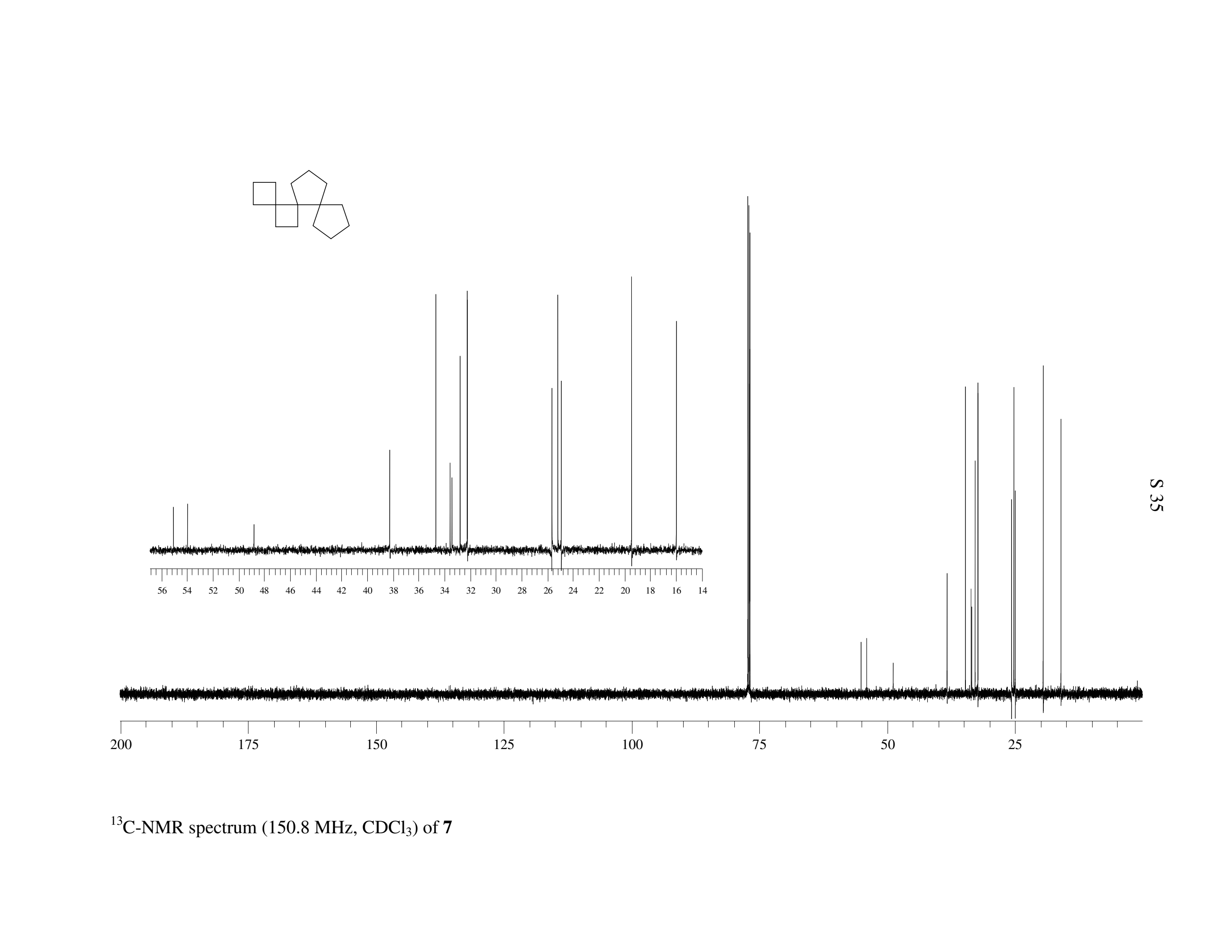




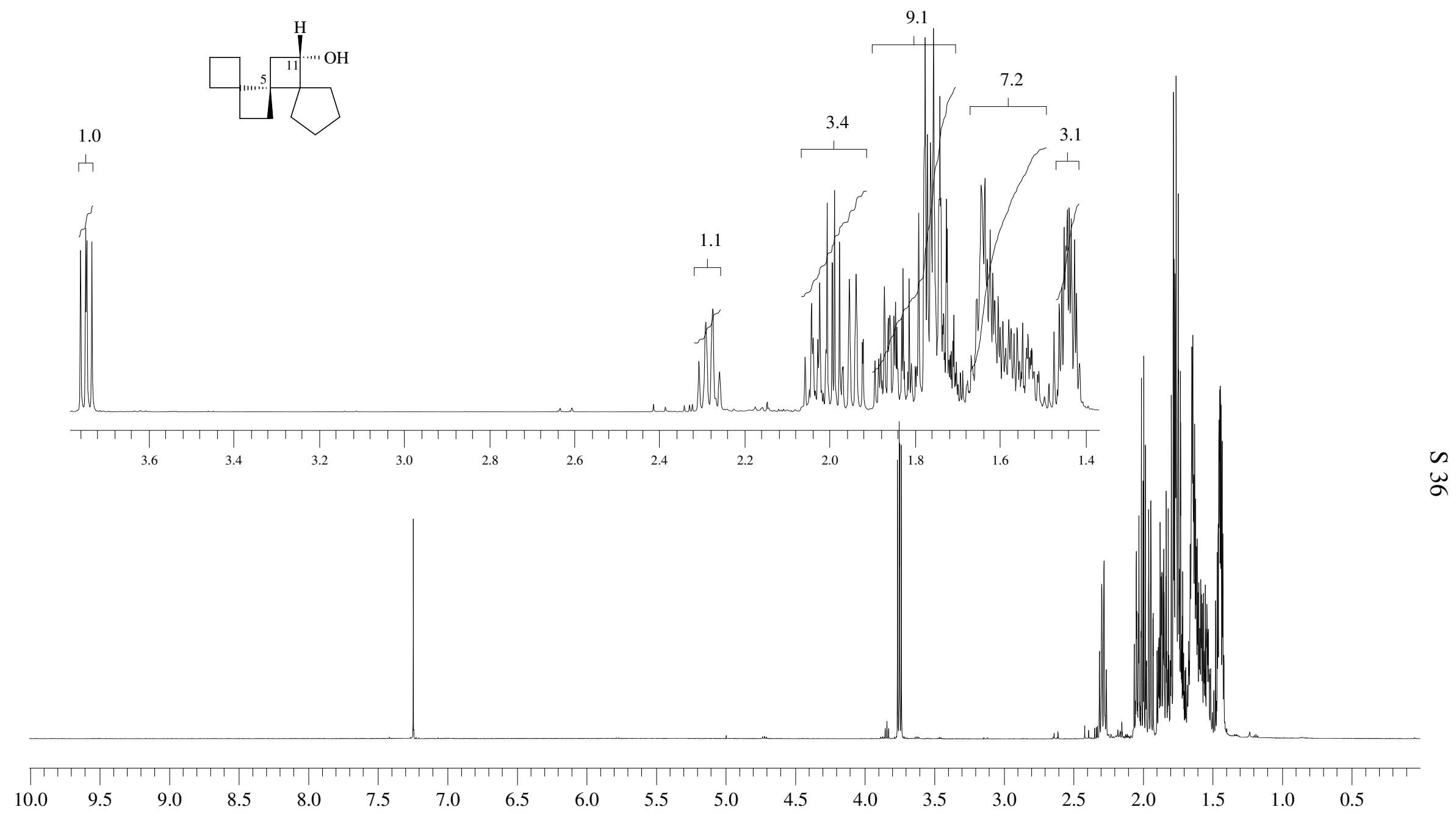

${ }^{1} \mathrm{H}-\mathrm{NMR}$ spectrum $\left(600 \mathrm{MHz}, \mathrm{CDCl}_{3}\right)$ of $(5 S, 11 S)-(+)-27$ 


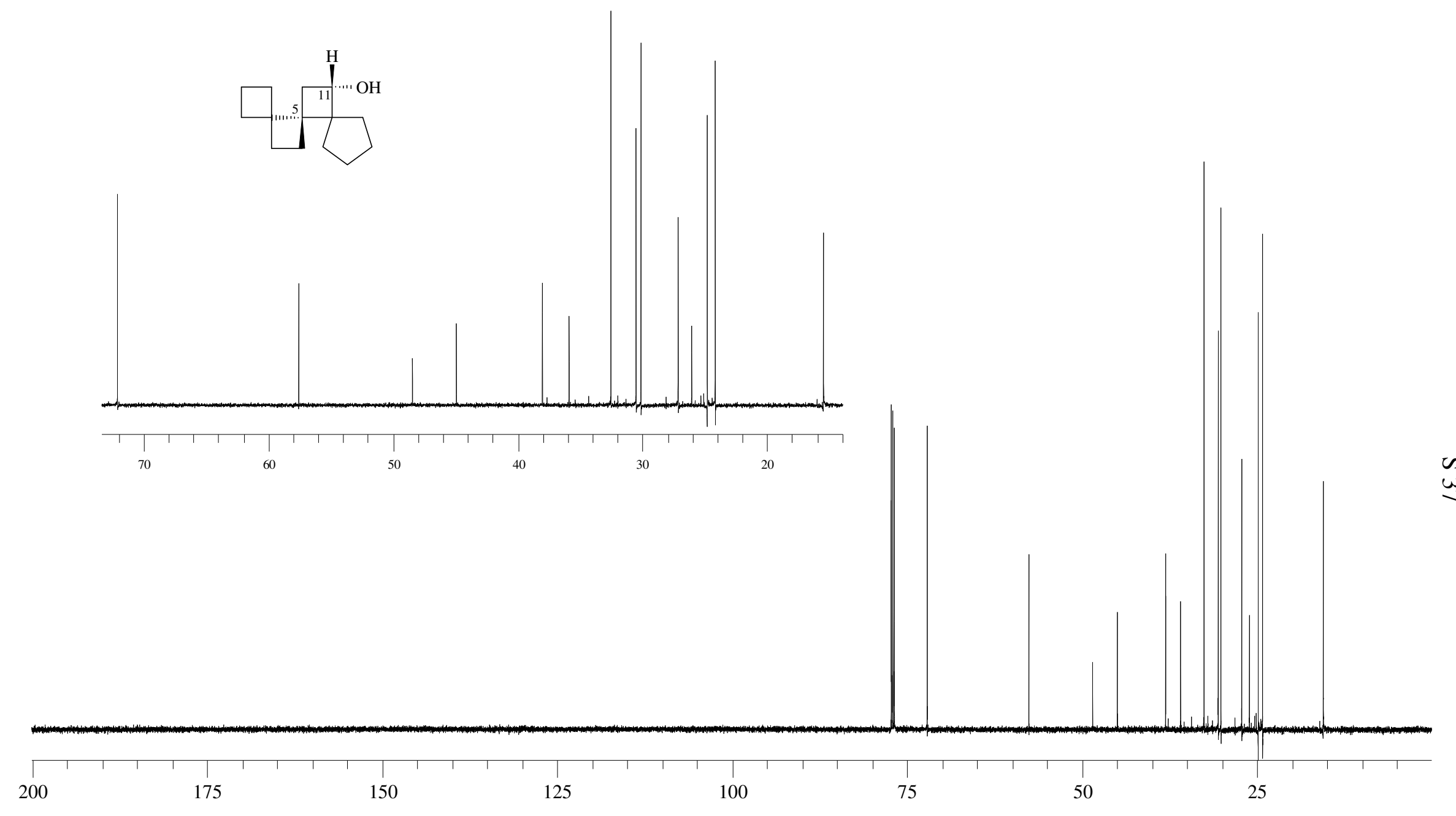

${ }^{13} \mathrm{C}-\mathrm{NMR}$ spectrum $\left(150.8 \mathrm{MHz}, \mathrm{CDCl}_{3}\right)$ of $(5 S, 11 S)-(+)-27$ 


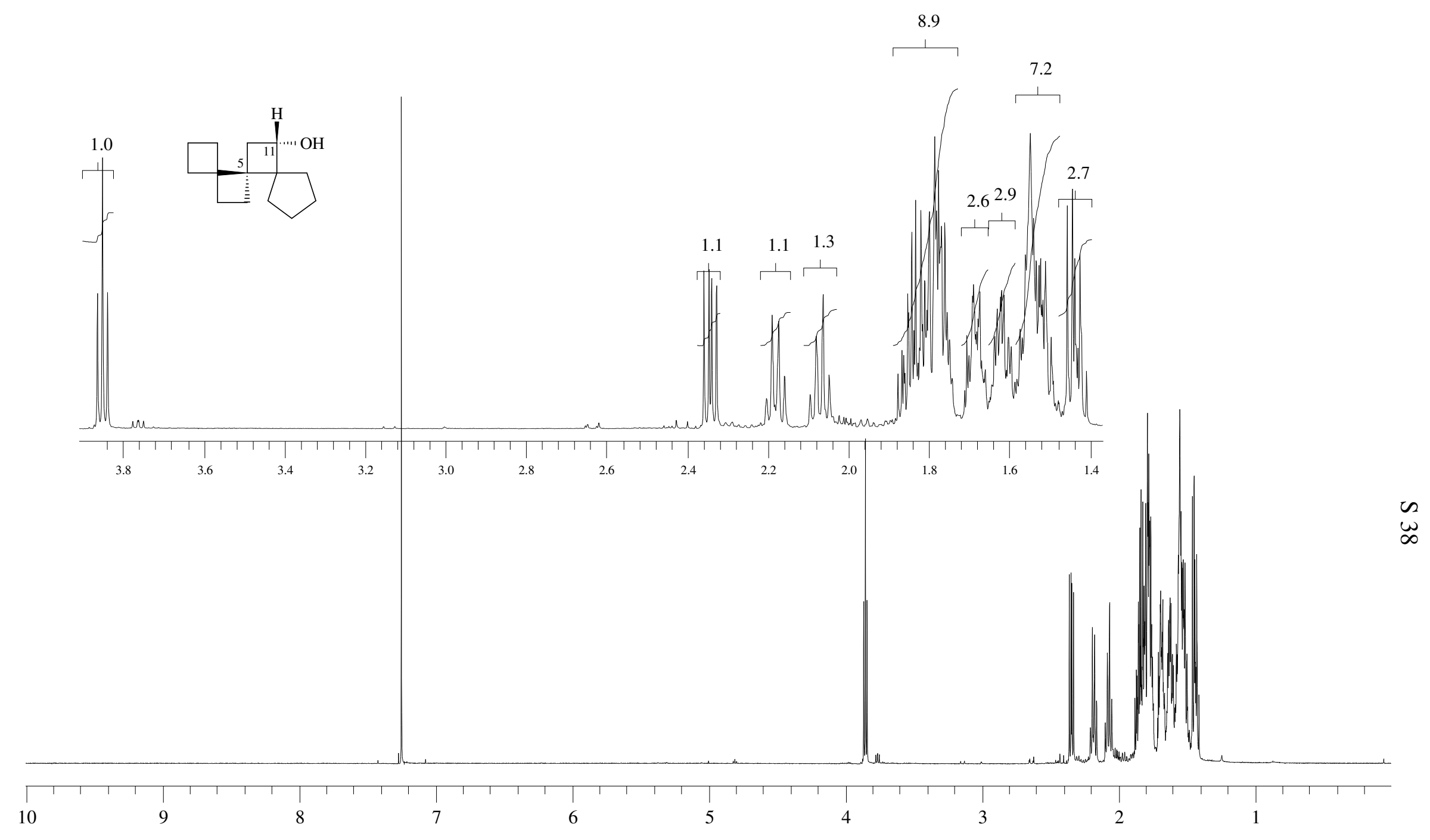

${ }^{1} \mathrm{H}-\mathrm{NMR}$ spectrum $\left(600 \mathrm{MHz}, \mathrm{CDCl}_{3}\right)$ of $(5 R, 11 S)-(+)-28$ 


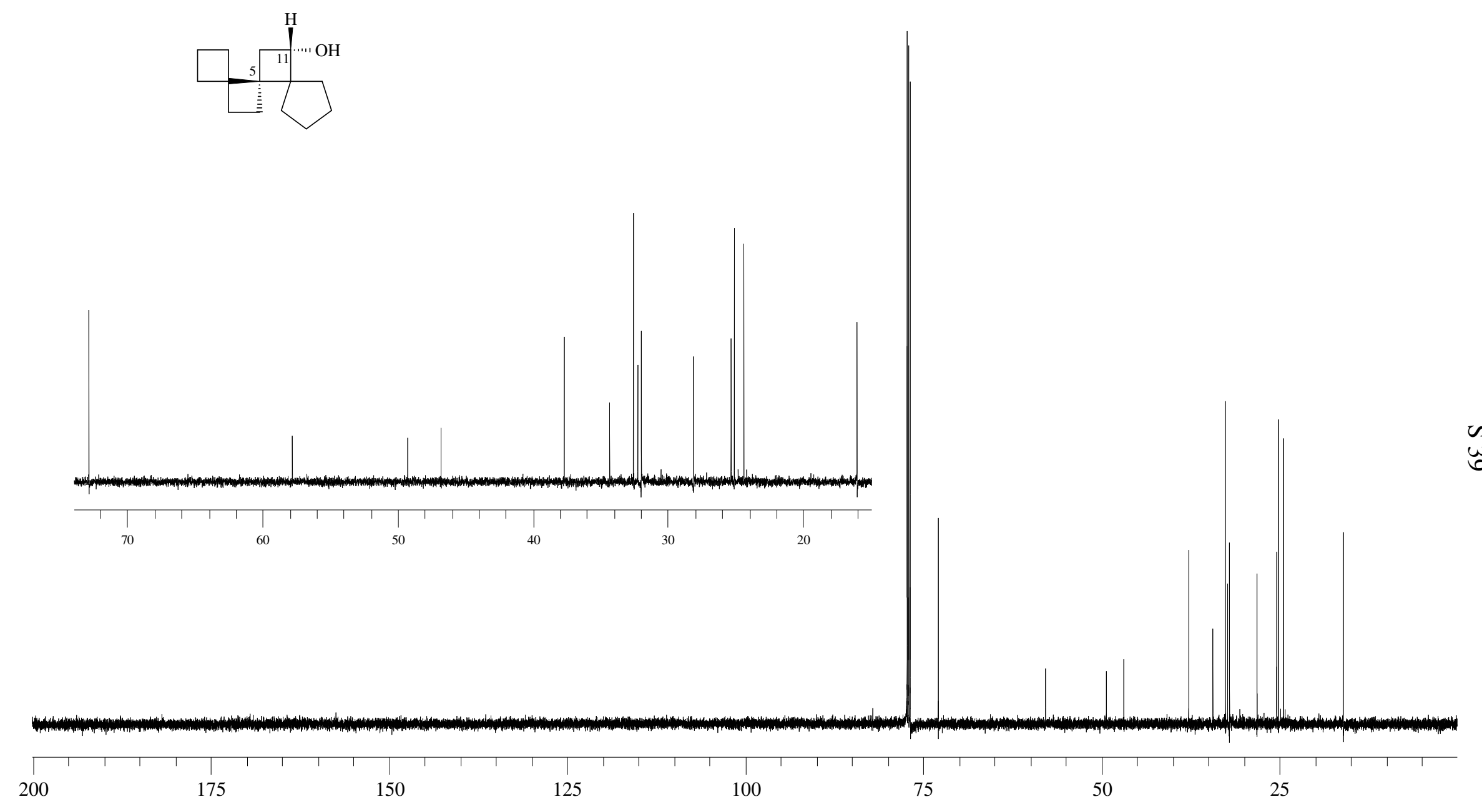

${ }^{13} \mathrm{C}-\mathrm{NMR}$ spectrum $\left(150.8 \mathrm{MHz}, \mathrm{CDCl}_{3}\right)$ of $(5 R, 11 S)-(+)-28$ 


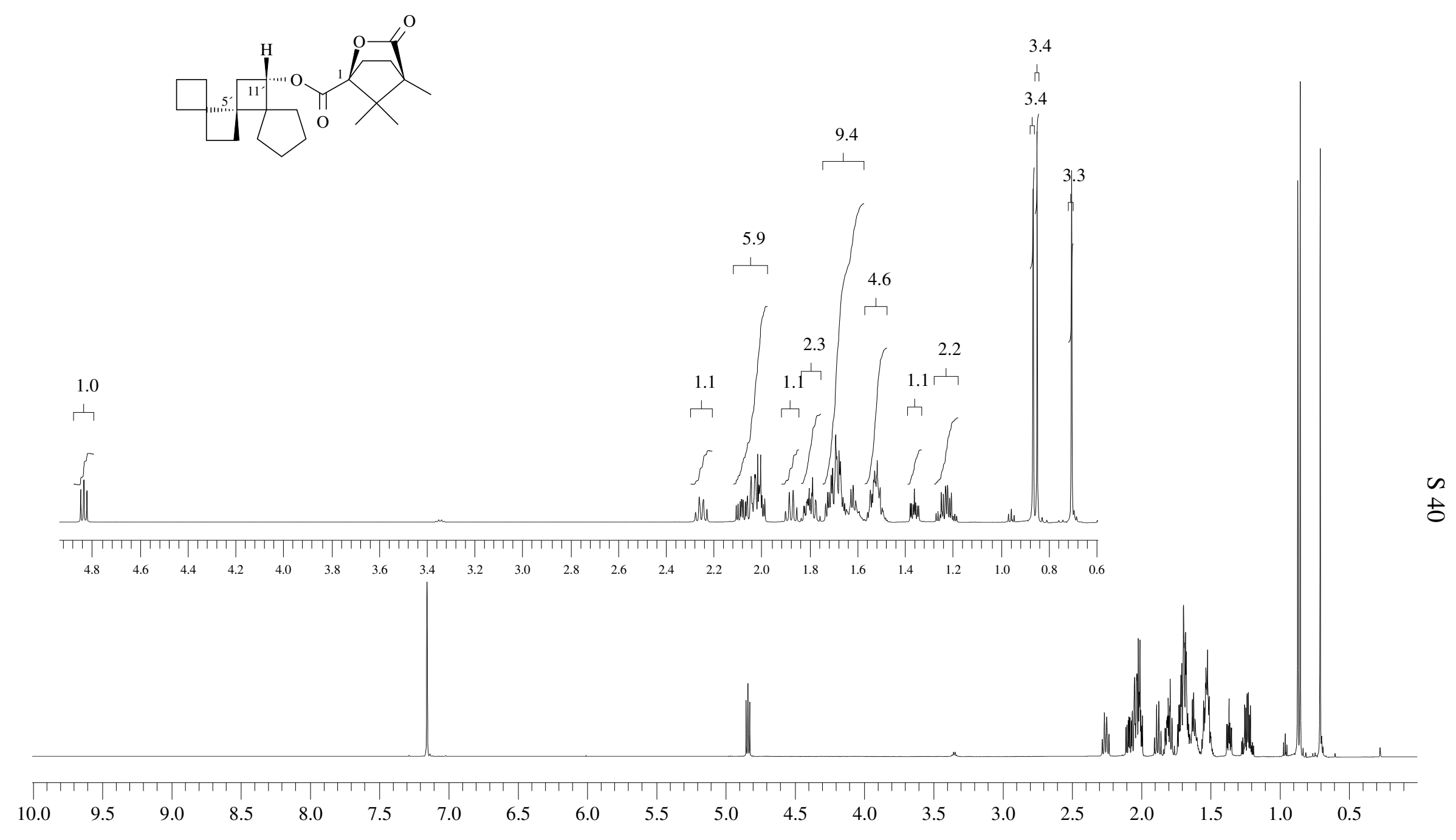

${ }^{1} \mathrm{H}-\mathrm{NMR}$ spectrum $\left(600 \mathrm{MHz}, \mathrm{C}_{6} \mathrm{D}_{6}\right)$ of $\left(1 S, 5^{\prime} S, 11^{\prime} S\right)-(+)-26$ 


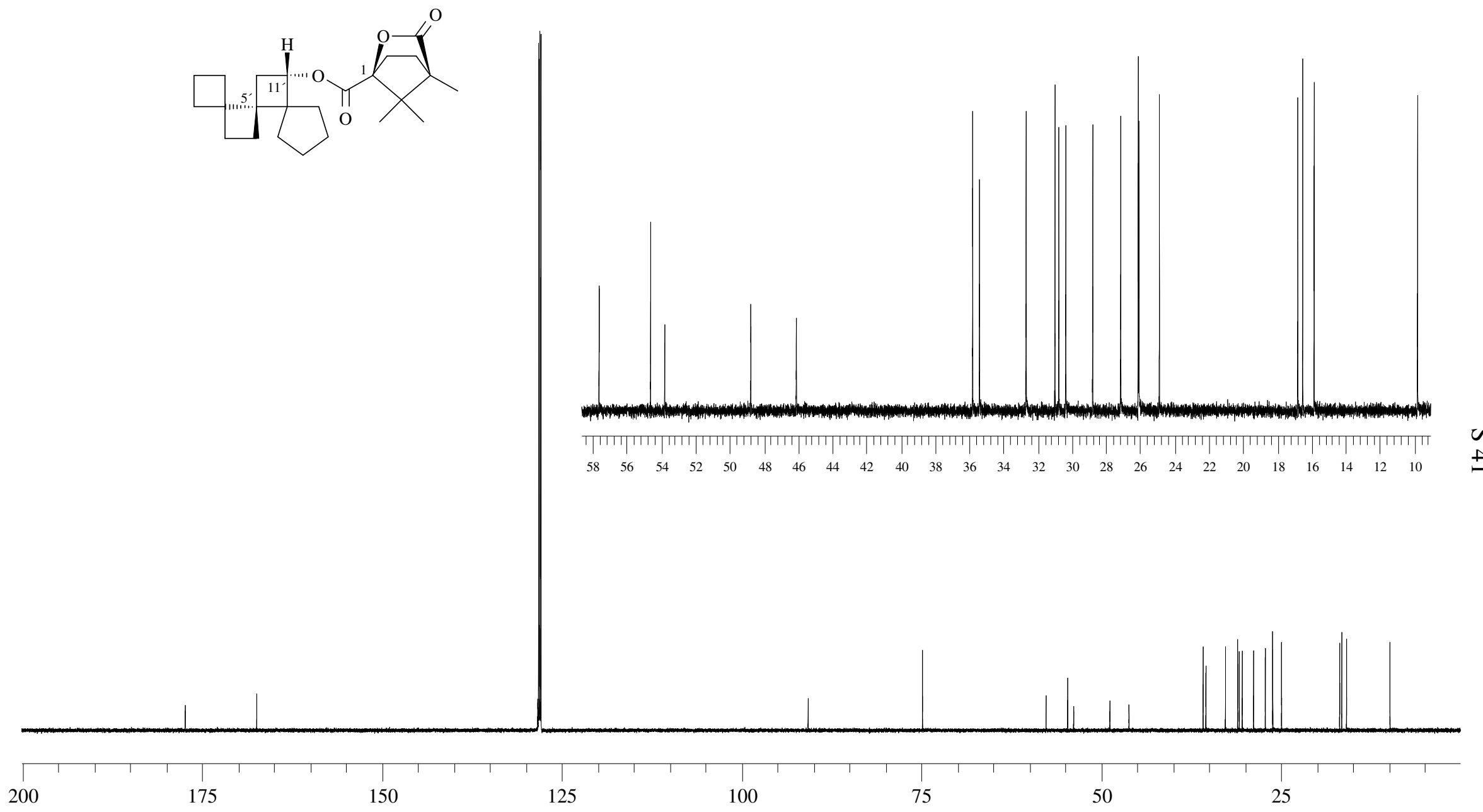

${ }^{13} \mathrm{C}-\mathrm{NMR}$ spectrum $\left(150.8 \mathrm{MHz}, \mathrm{C}_{6} \mathrm{D}_{6}\right)$ of $\left(1 S, 5^{\prime} S, 11^{\prime} S\right)-(+)-26$ 


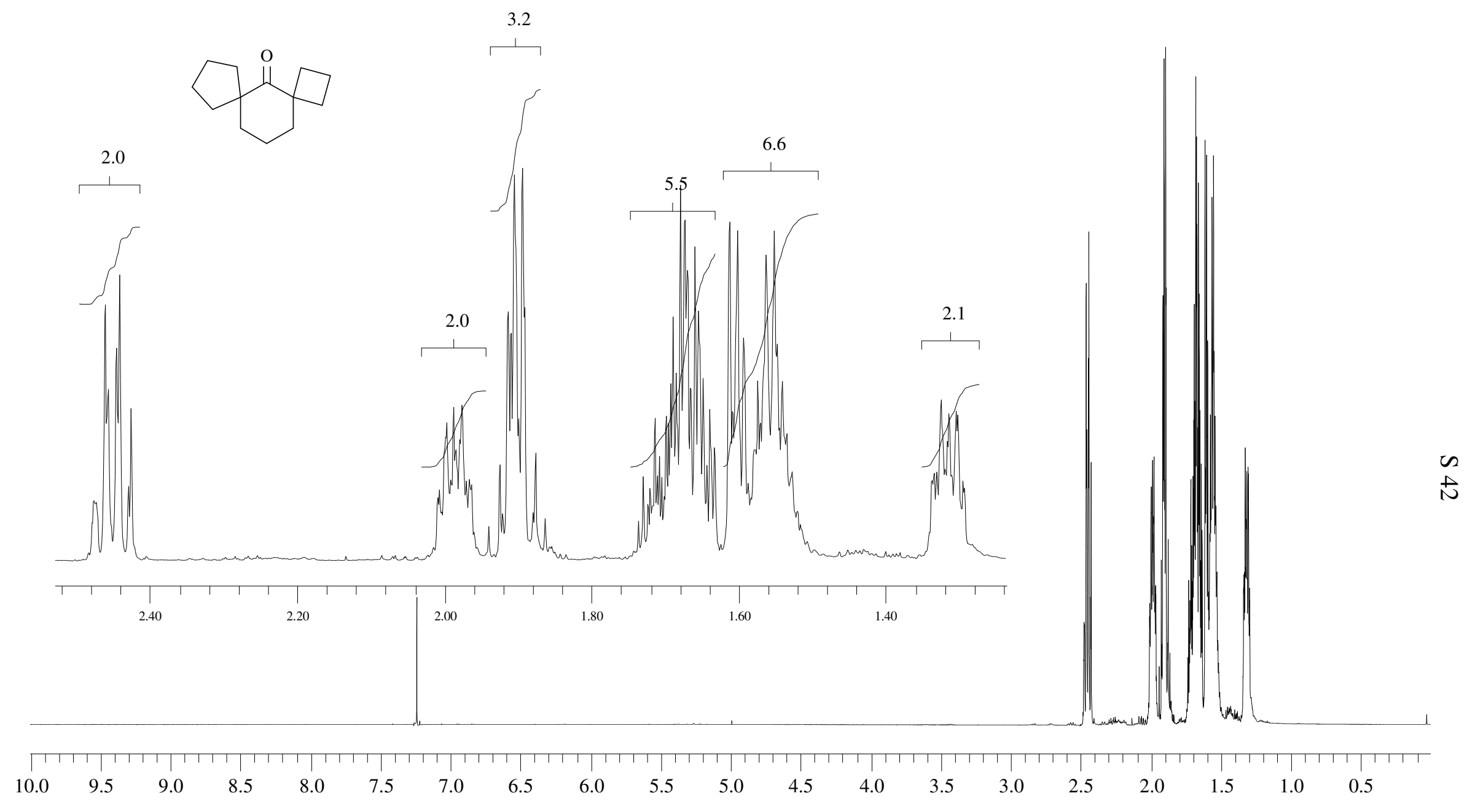

${ }^{1} \mathrm{H}$-NMR spectrum $\left(600 \mathrm{MHz}, \mathrm{CDCl}_{3}\right.$ ) of $\mathbf{3 1}$ 

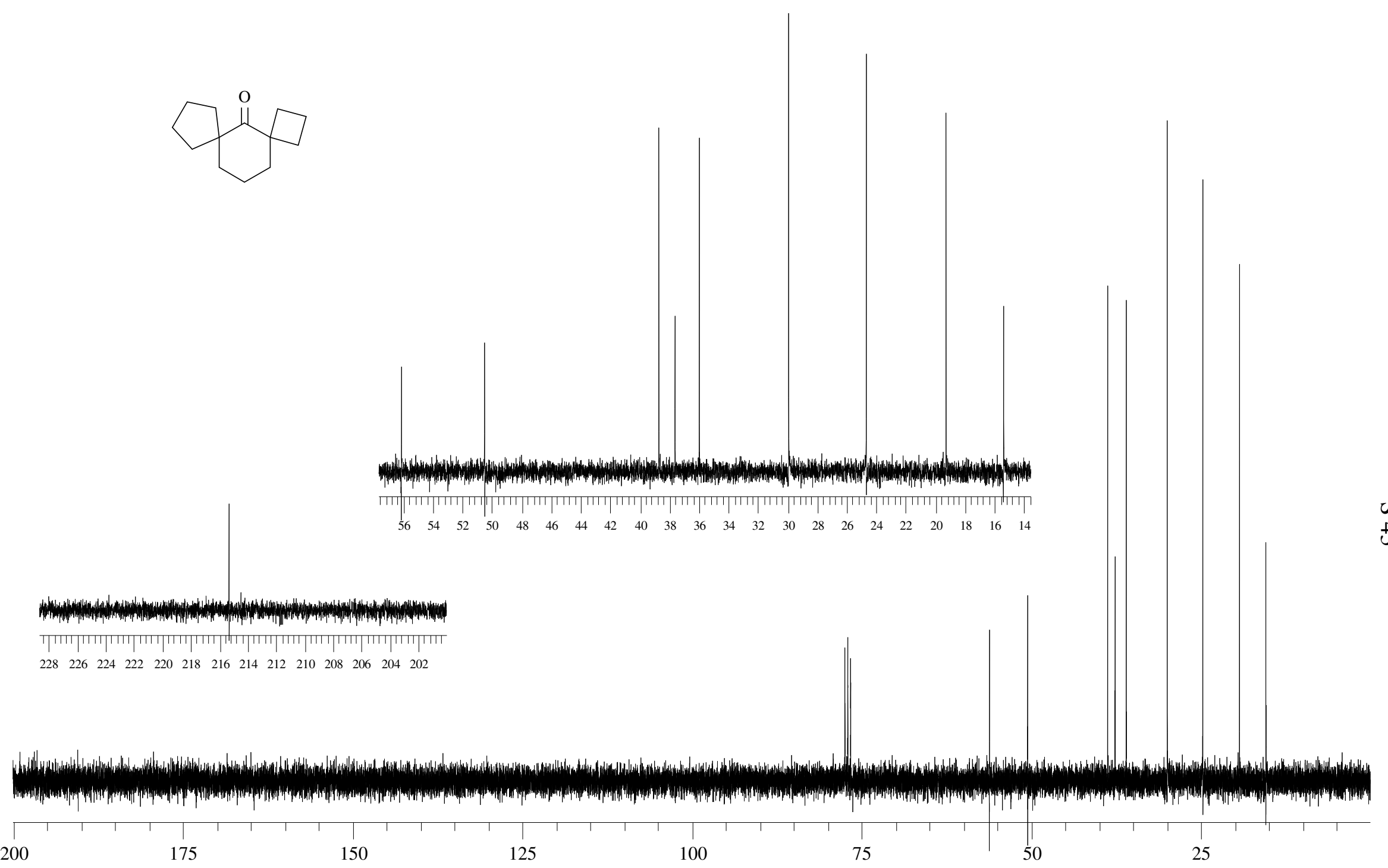

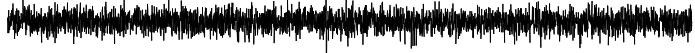

Tा

$\begin{array}{llllllllllllll}228 & 226 & 224 & 222 & 220 & 218 & 216 & 214 & 212 & 210 & 208 & 206 & 204 & 202\end{array}$

${ }^{13} \mathrm{C}$-NMR spectrum $\left(75.5 \mathrm{MHz}, \mathrm{CDCl}_{3}\right)$ of $\mathbf{3 1}$ 


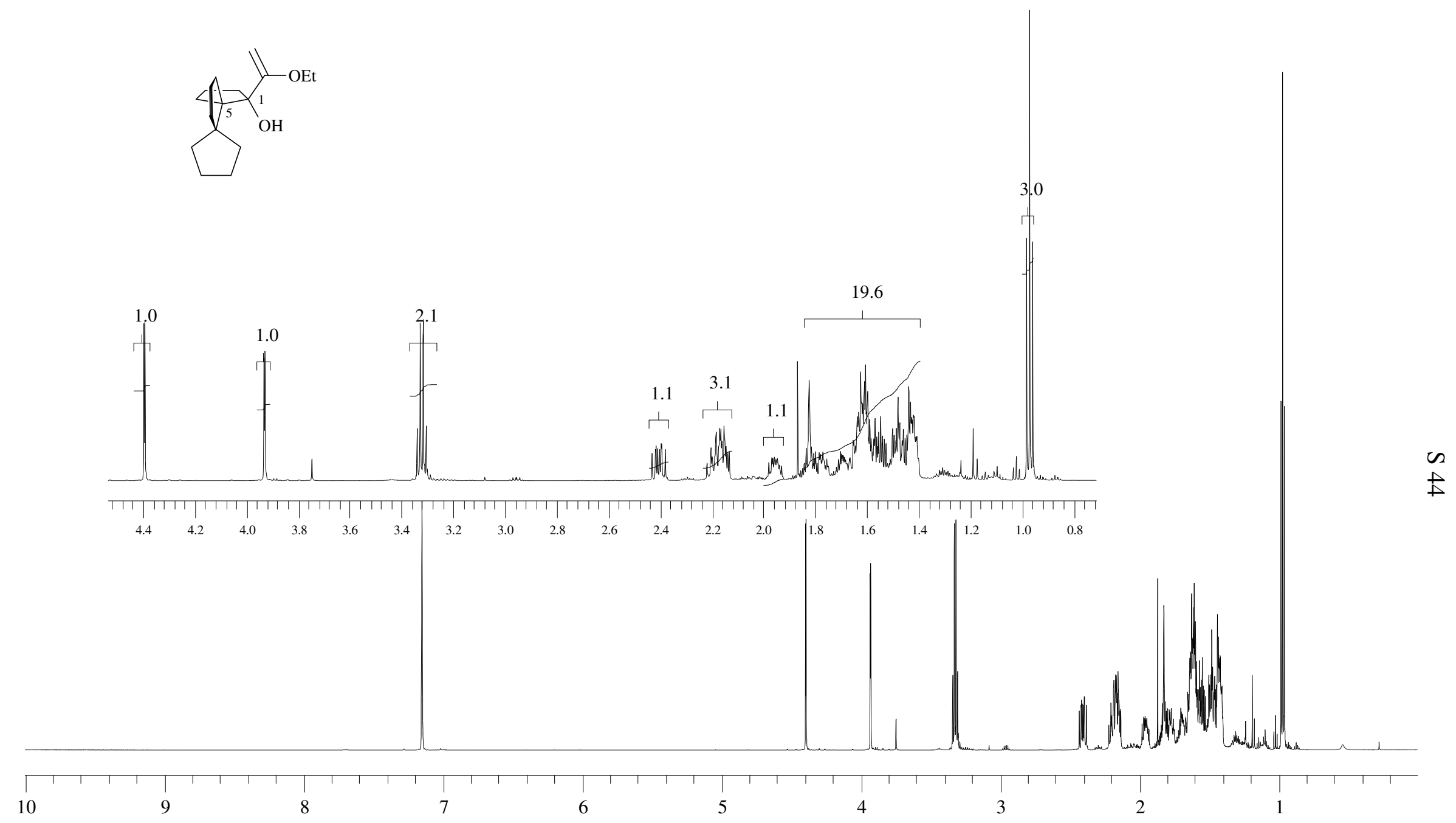

${ }^{1} \mathrm{H}-\mathrm{NMR}$ spectrum $\left(600 \mathrm{MHz}, \mathrm{C}_{6} \mathrm{D}_{6}\right)$ of $\left(1 S^{*}, 5 S^{*}\right)-\mathbf{3 3}$ 


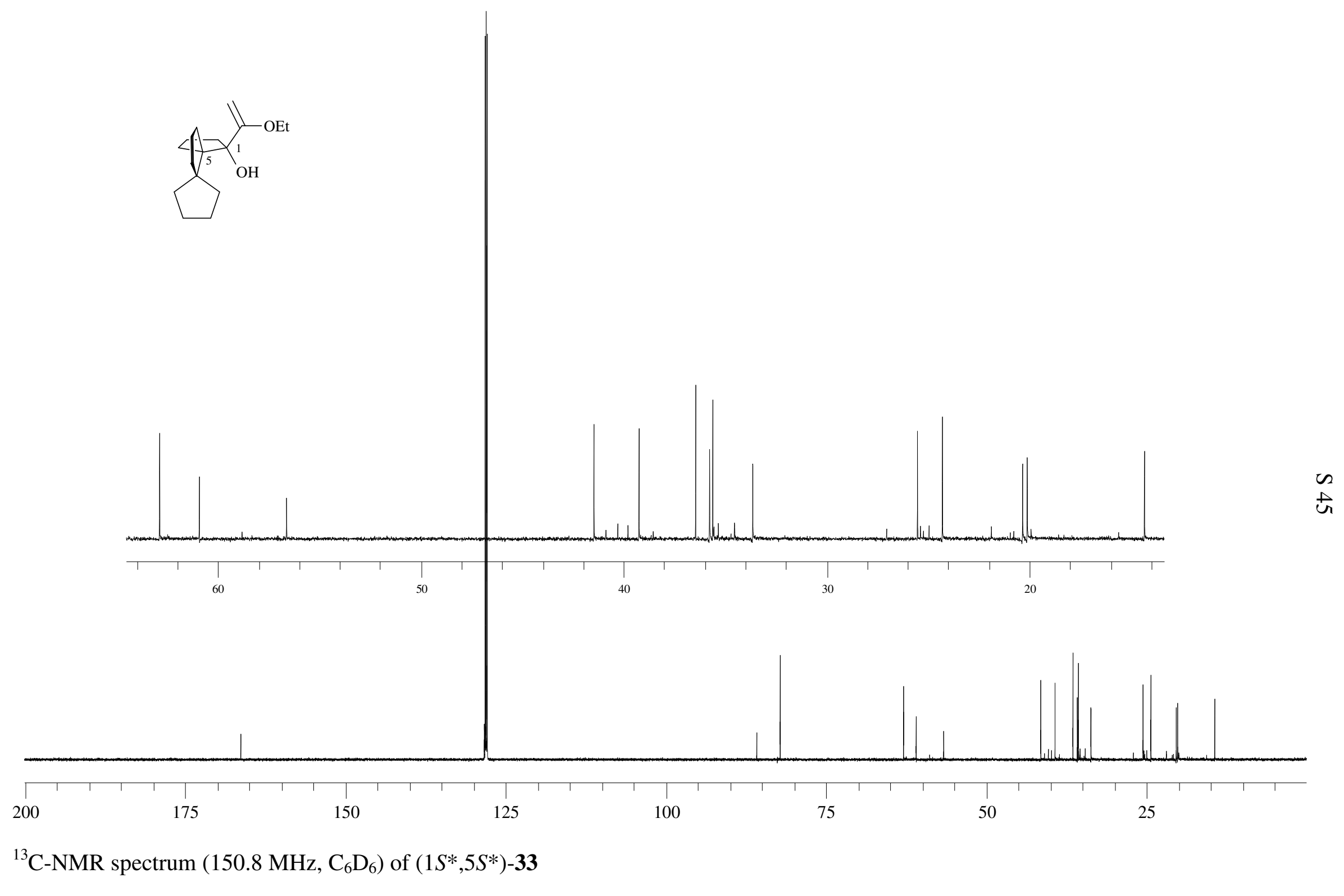




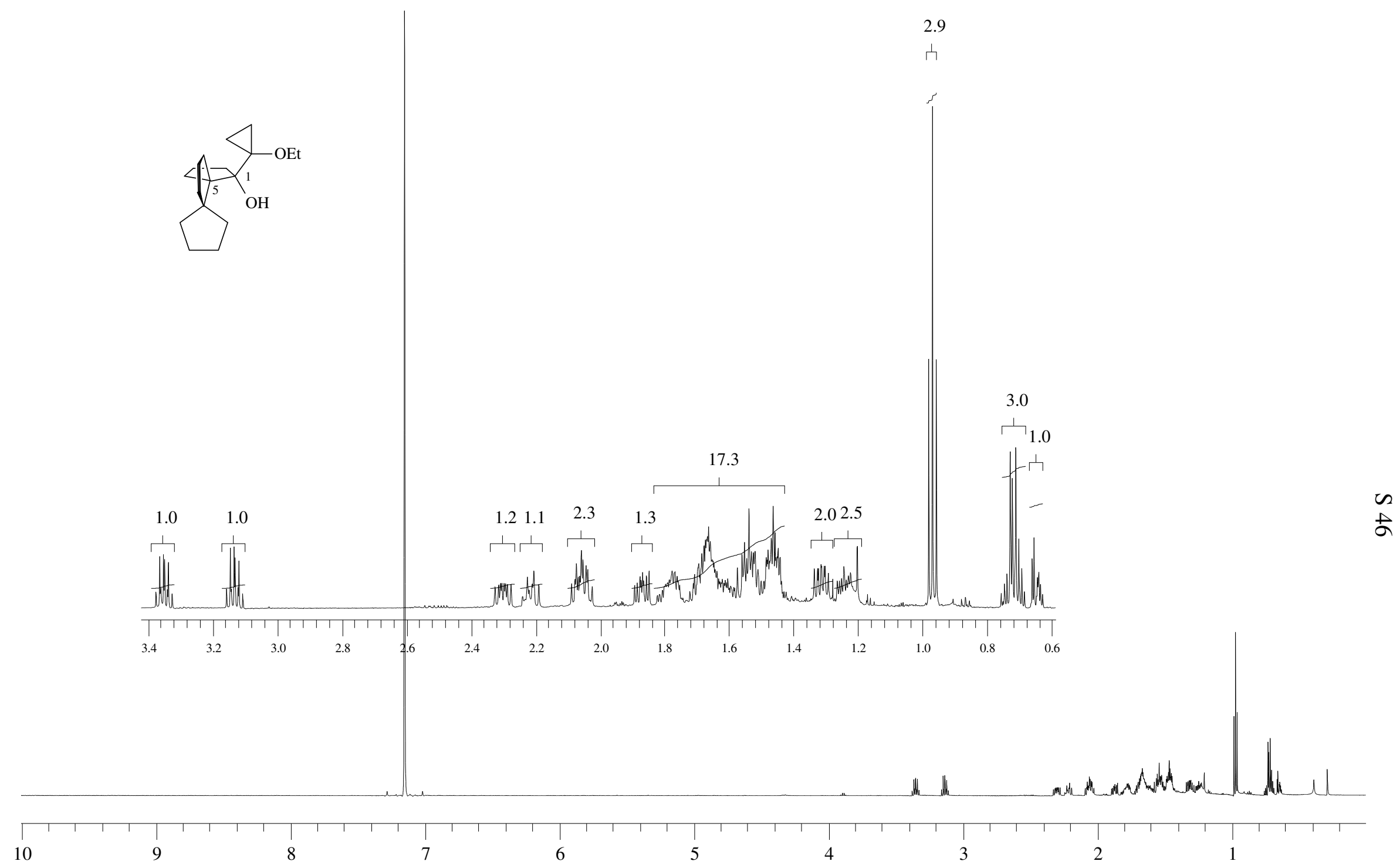

${ }^{1} \mathrm{H}-\mathrm{NMR}$ spectrum $\left(600 \mathrm{MHz}, \mathrm{C}_{6} \mathrm{D}_{6}\right)$ of $\left(1 S^{*}, 5 S^{*}\right)-35$ 


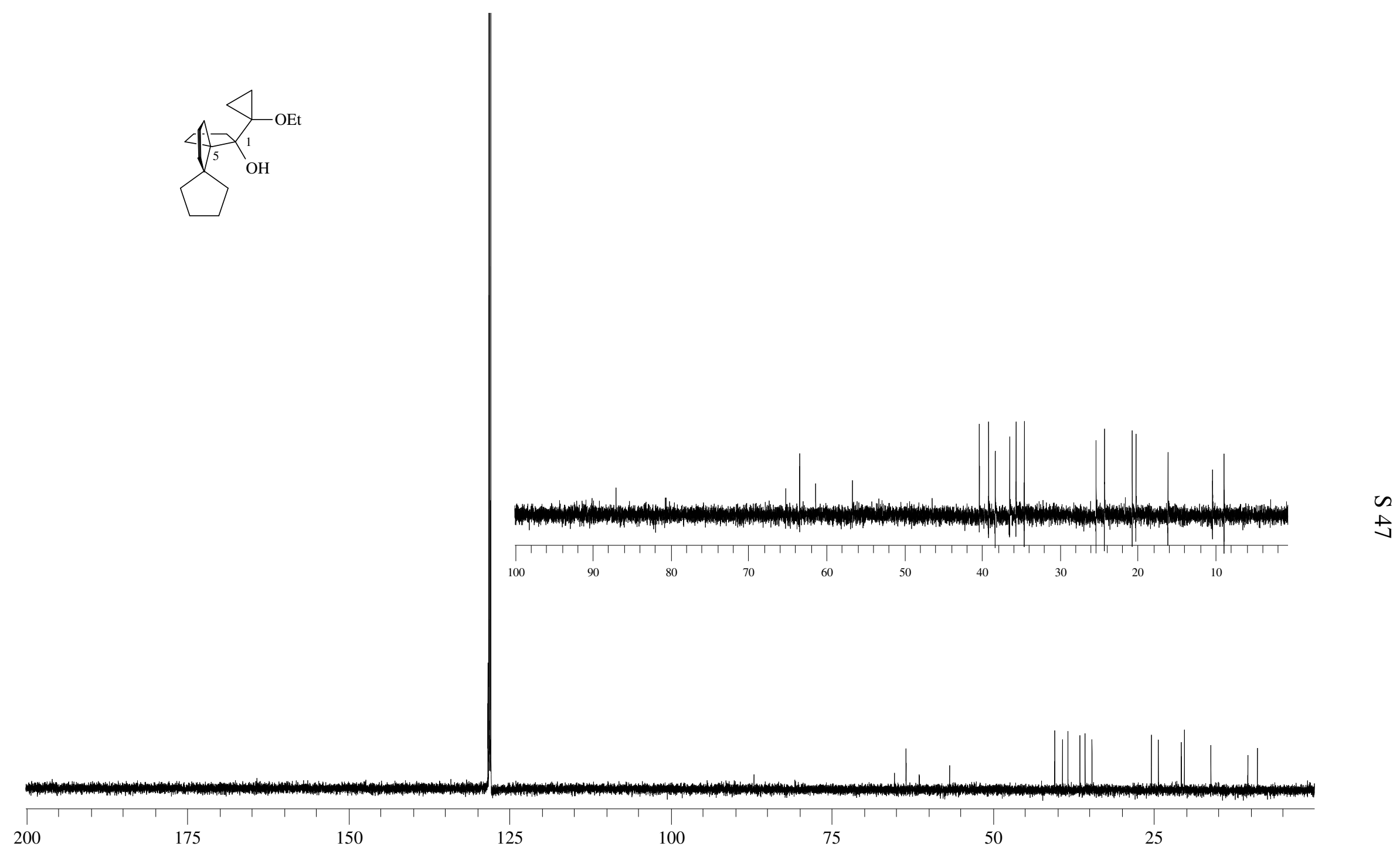

${ }^{13} \mathrm{C}$-NMR spectrum $\left(150.8 \mathrm{MHz}, \mathrm{C}_{6} \mathrm{D}_{6}\right)$ of $\left(1 S^{*}, 5 S^{*}\right)-35$ 


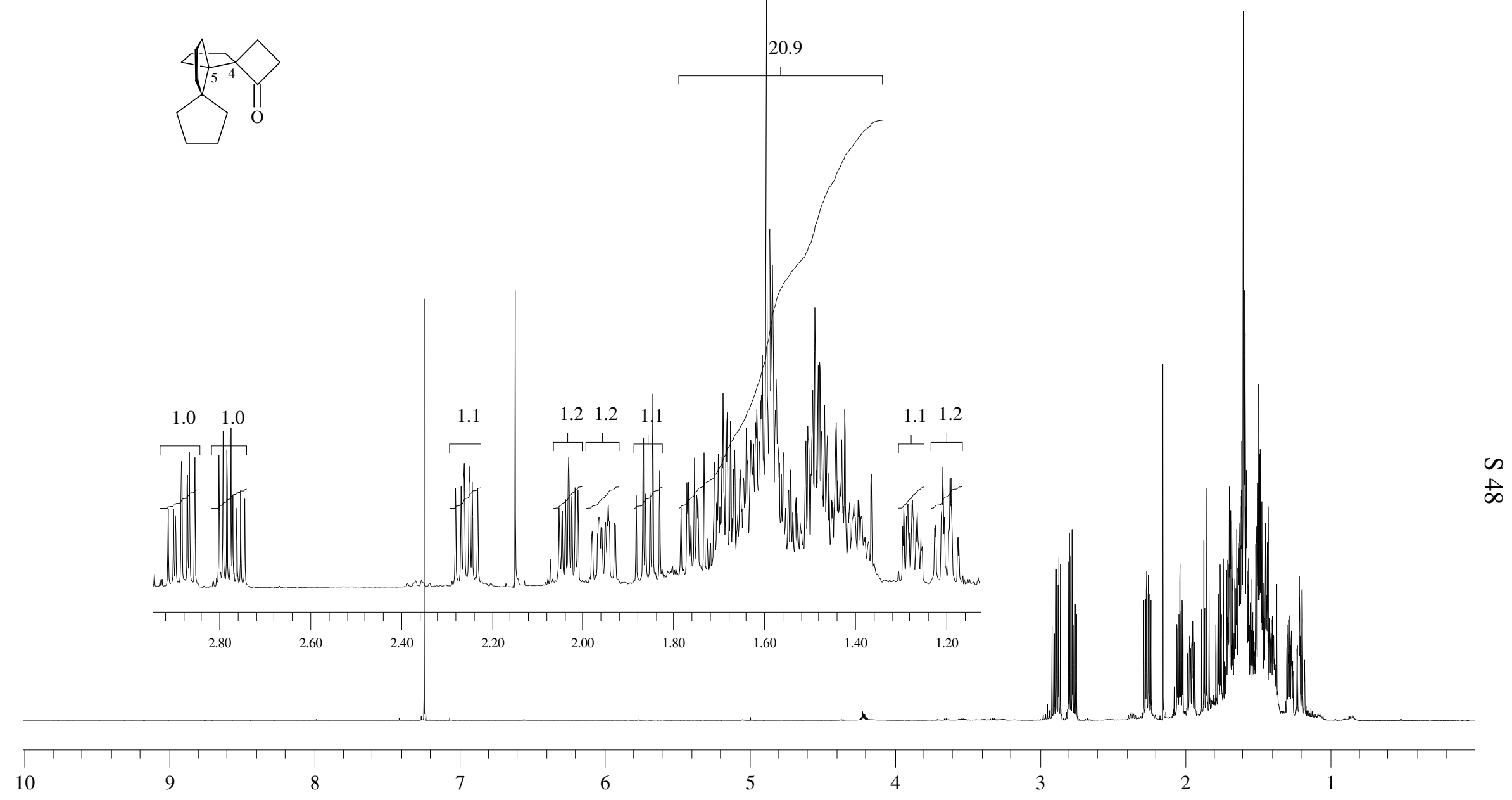

${ }^{1} \mathrm{H}-\mathrm{NMR}$ spectrum $\left(600 \mathrm{MHz}, \mathrm{CDCl}_{3}\right)$ of $\left(4 R^{*}, 5 S^{*}\right)-\mathbf{3 6}$ 

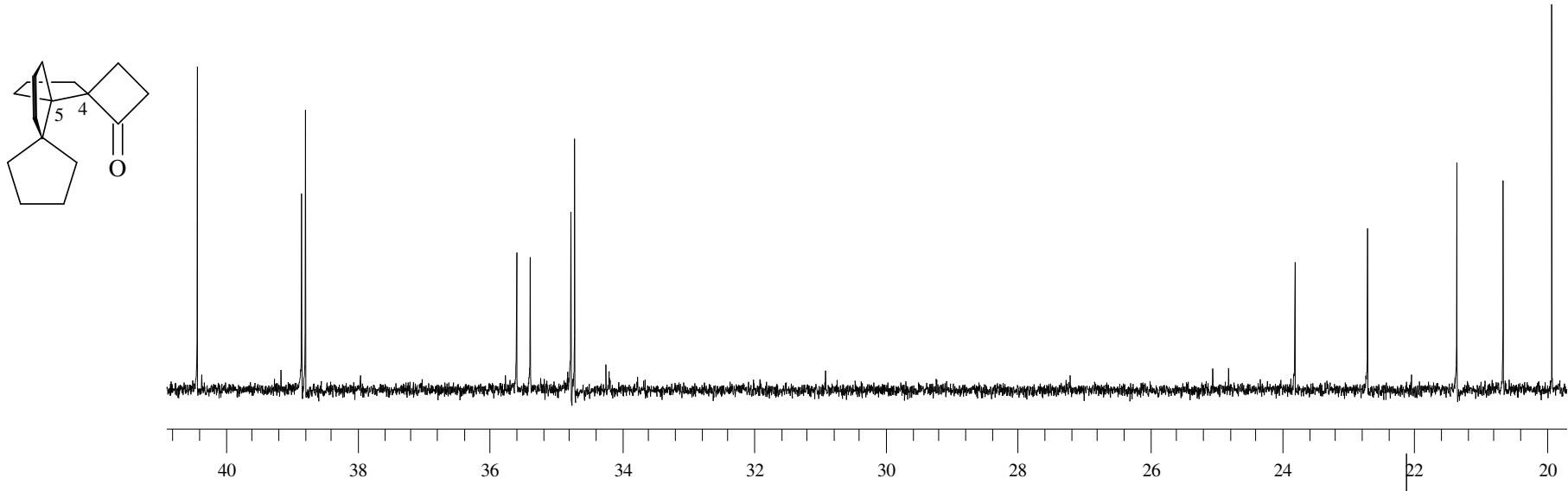

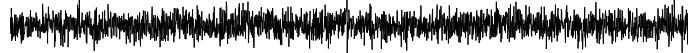

प

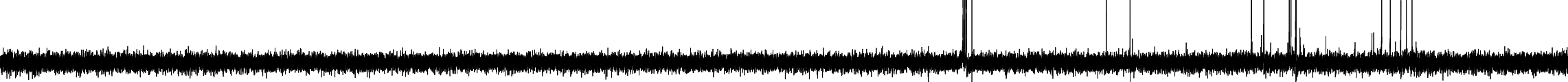

${ }^{13} \mathrm{C}$-NMR spectrum $\left(150.8 \mathrm{MHz}, \mathrm{CDCl}_{3}\right)$ of $\left(4 R^{*}, 5 S^{*}\right)-36$ 


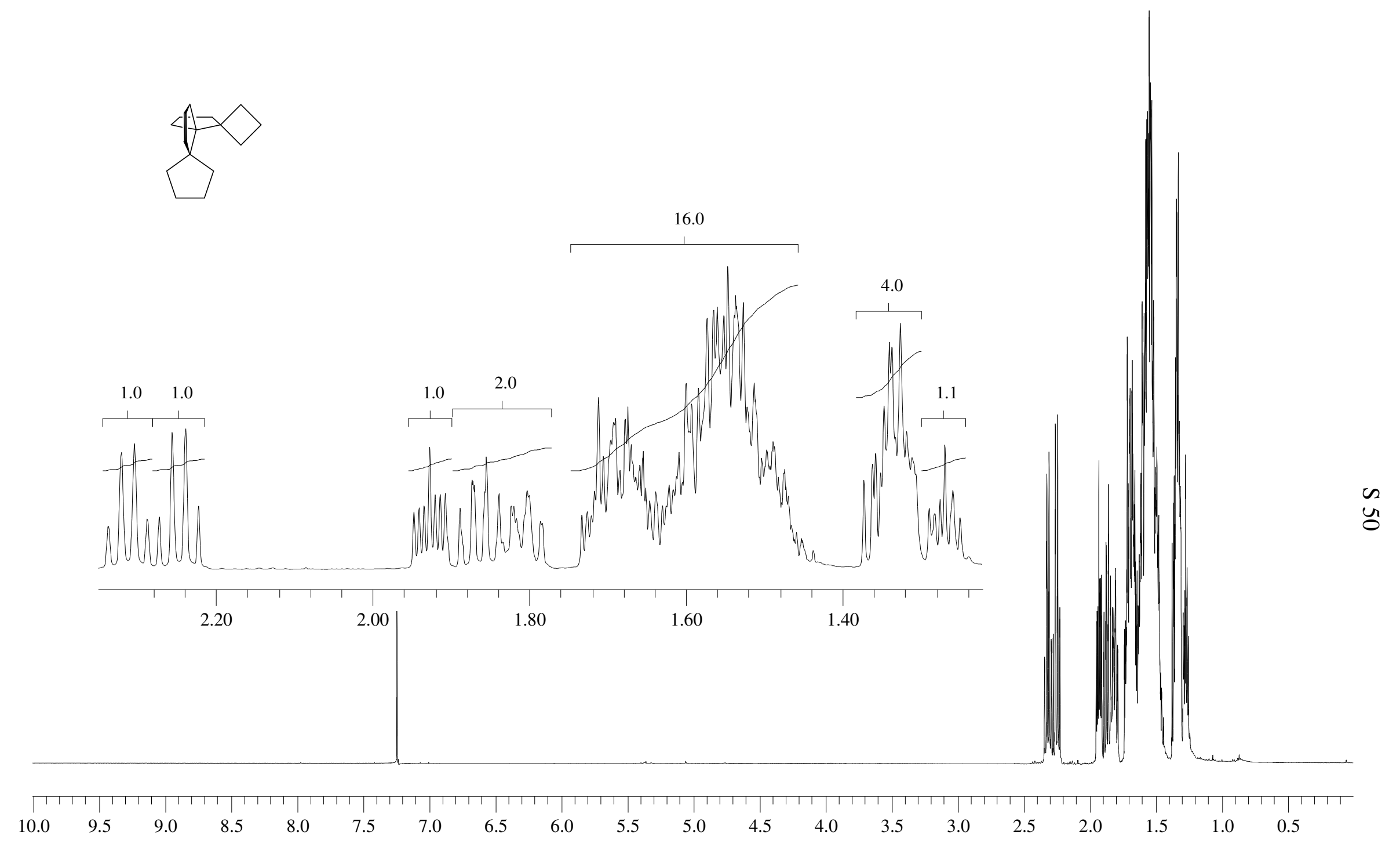

${ }^{1} \mathrm{H}-\mathrm{NMR}$ spectrum $\left(600 \mathrm{MHz}, \mathrm{CDCl}_{3}\right)$ of $\left(5 S^{*}\right)-8$ 

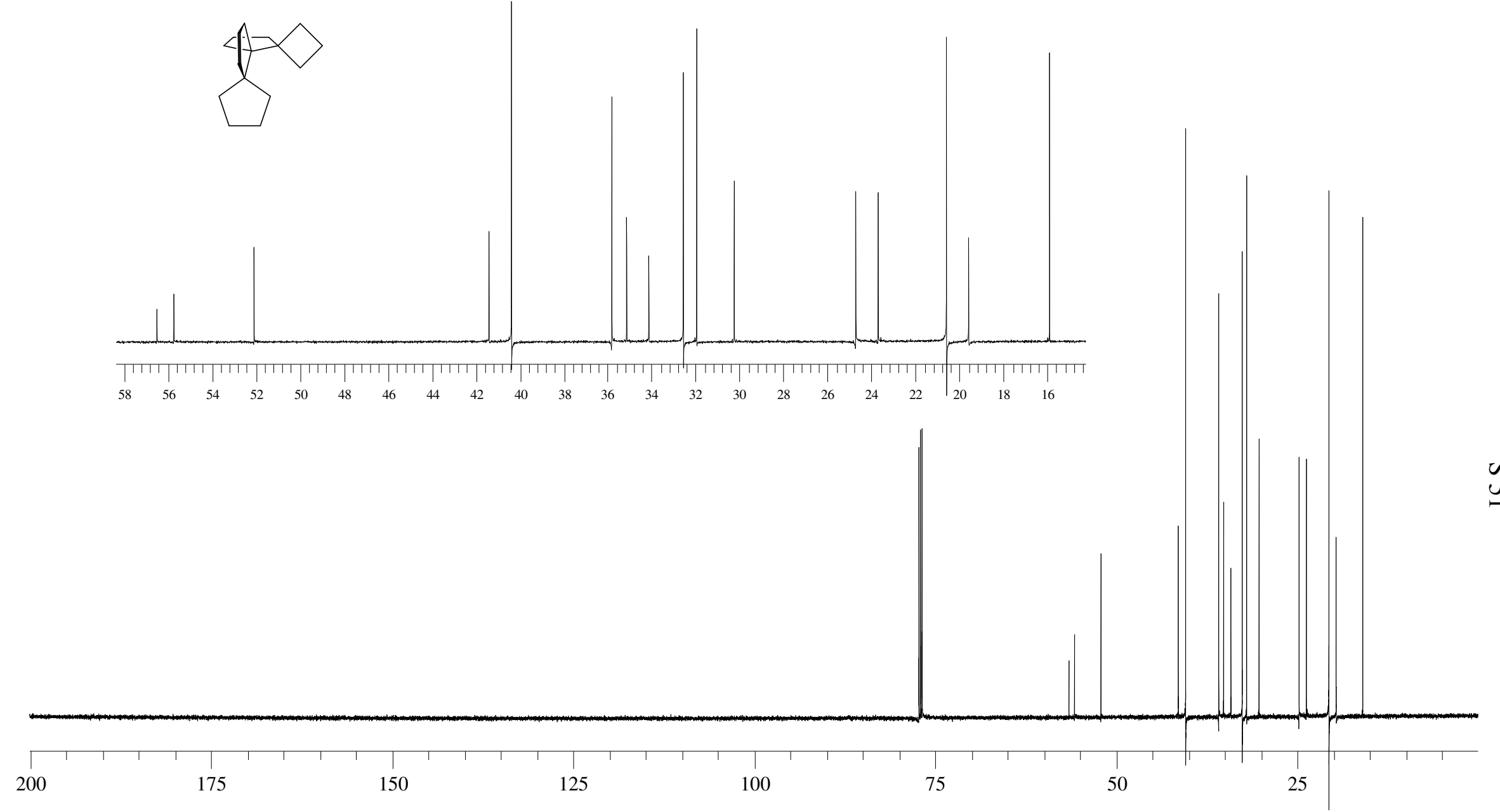

${ }^{13} \mathrm{C}-\mathrm{NMR}$ spectrum $\left(150.8 \mathrm{MHz}, \mathrm{CDCl}_{3}\right)$ of $\left(5 S^{*}\right)-8$ 


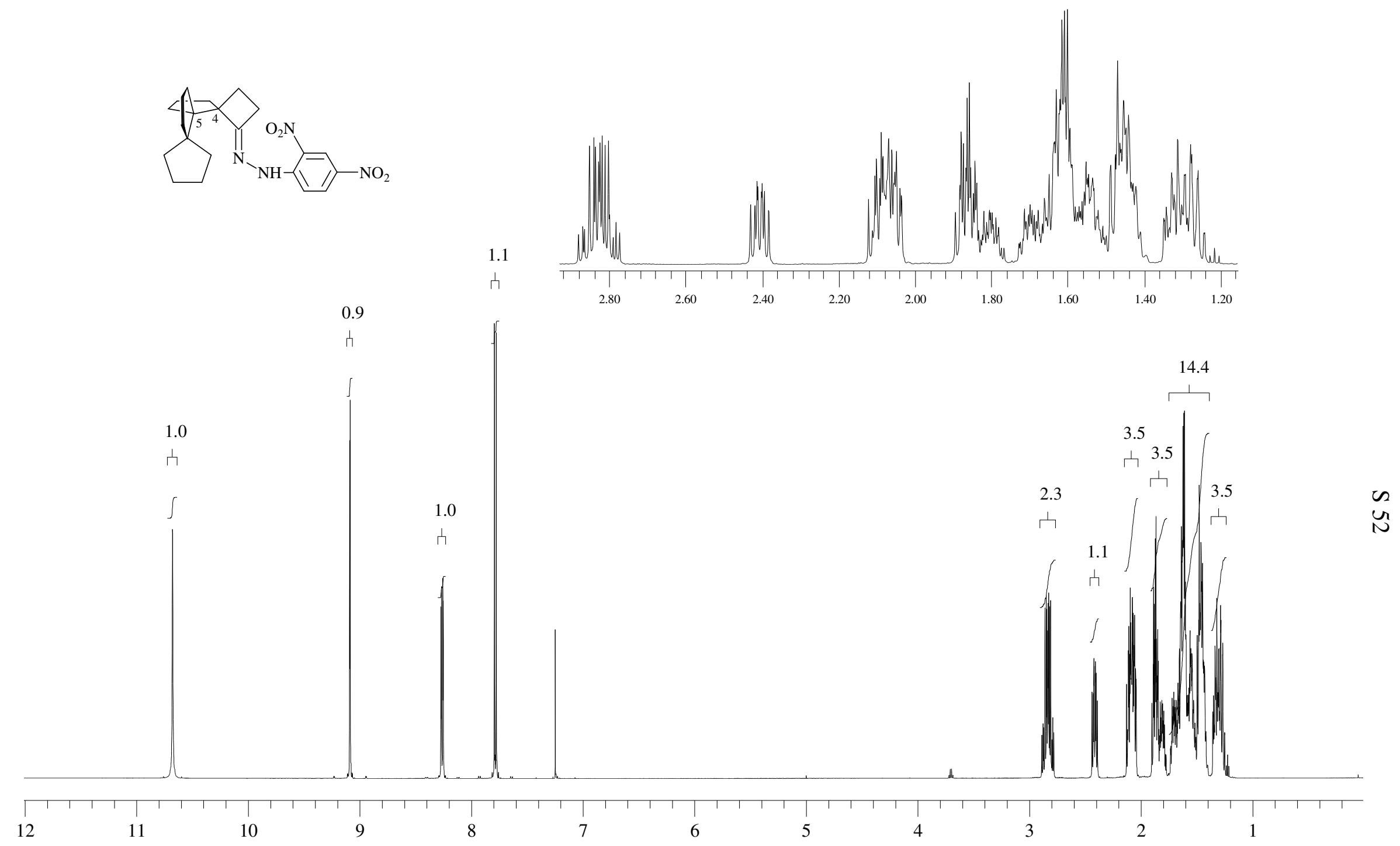

${ }^{1} \mathrm{H}-\mathrm{NMR}$ spectrum $\left(600 \mathrm{MHz}, \mathrm{CDCl}_{3}\right)$ of $\left(4 R^{*}, 5 S^{*}\right)-\mathbf{3 4}$ 


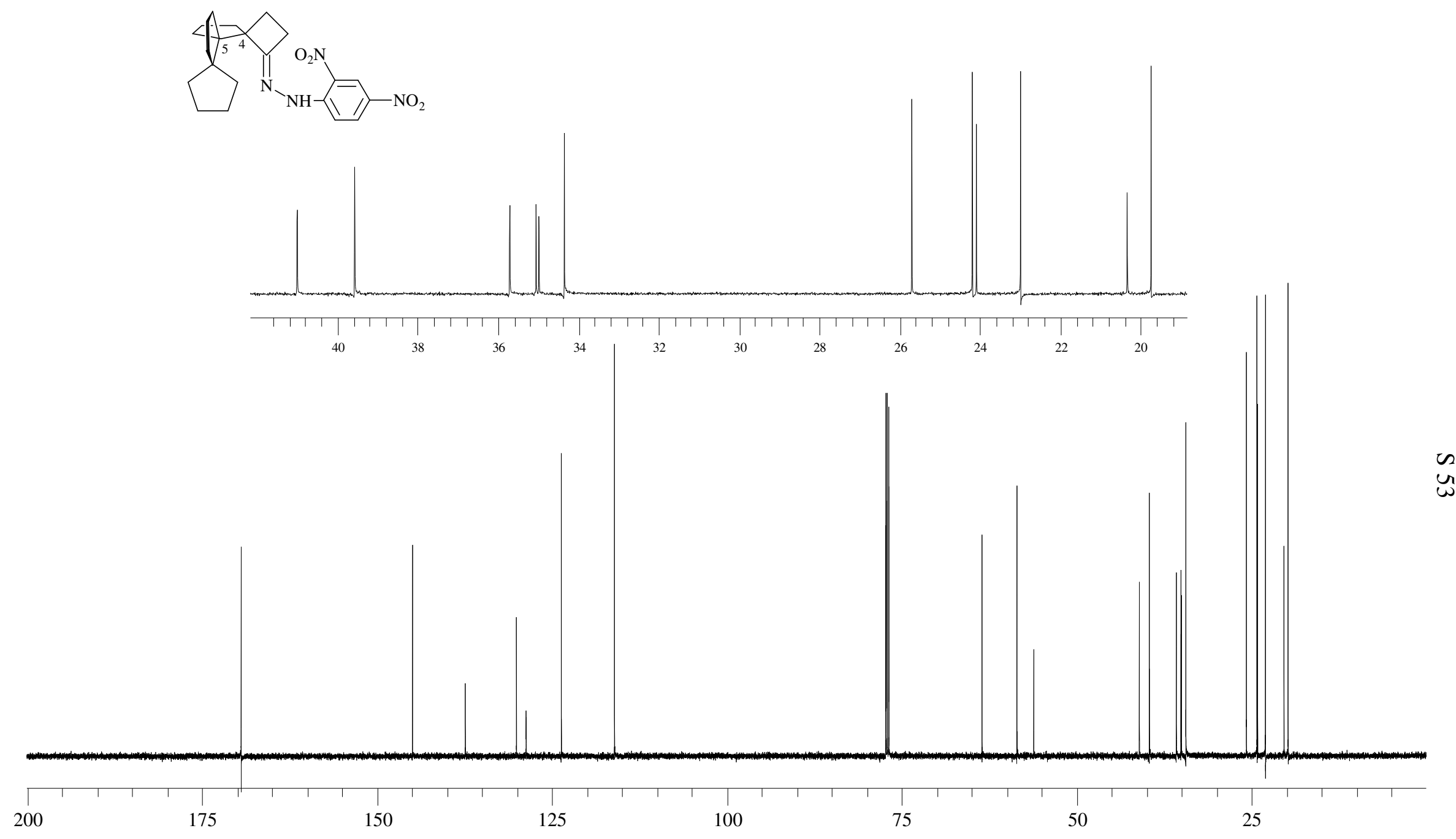

${ }^{13} \mathrm{C}-\mathrm{NMR}$ spectrum $\left(150.8 \mathrm{MHz}, \mathrm{CDCl}_{3}\right)$ of $\left(4 R^{*}, 5 S^{*}\right)-34$ 


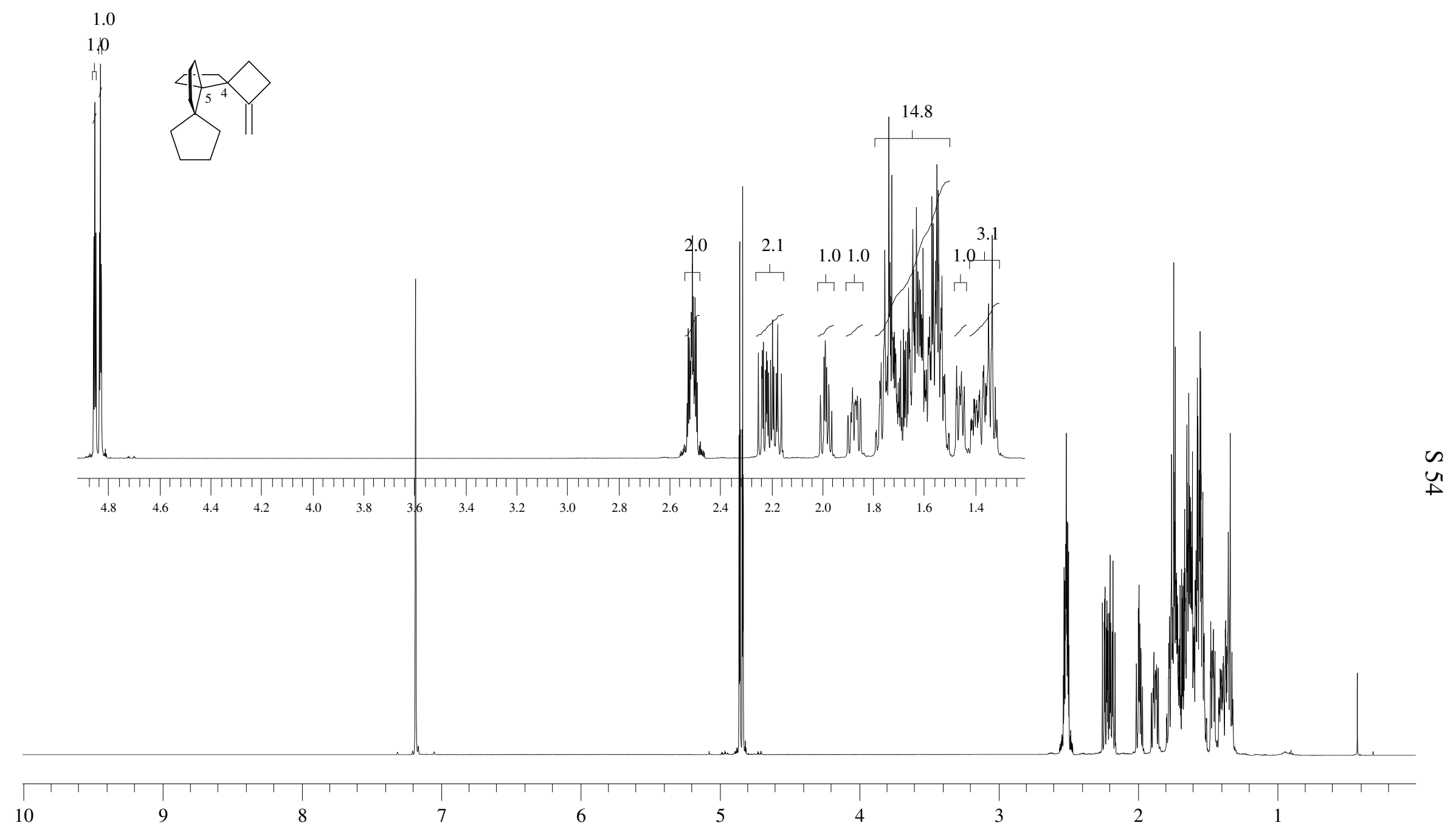

${ }^{1} \mathrm{H}-\mathrm{NMR}$ spectrum $\left(600 \mathrm{MHz}, \mathrm{C}_{6} \mathrm{D}_{6}\right)$ of $\left(4 R^{*}, 5 S^{*}\right)-\mathbf{3 7}$ 


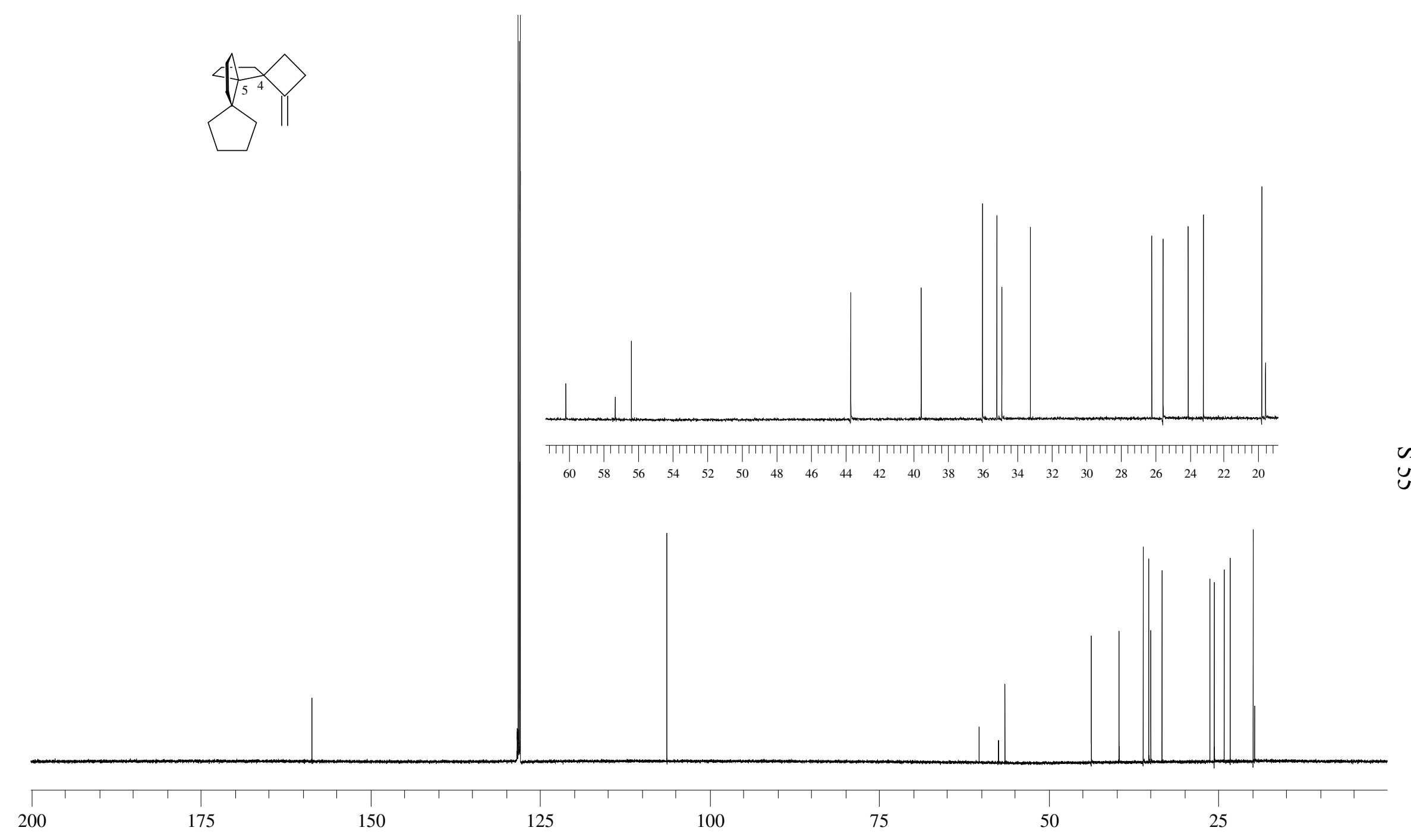

${ }^{13} \mathrm{C}$-NMR spectrum $\left(150.8 \mathrm{MHz}, \mathrm{C}_{6} \mathrm{D}_{6}\right)$ of $\left(4 R^{*}, 5 S^{*}\right)-37$ 


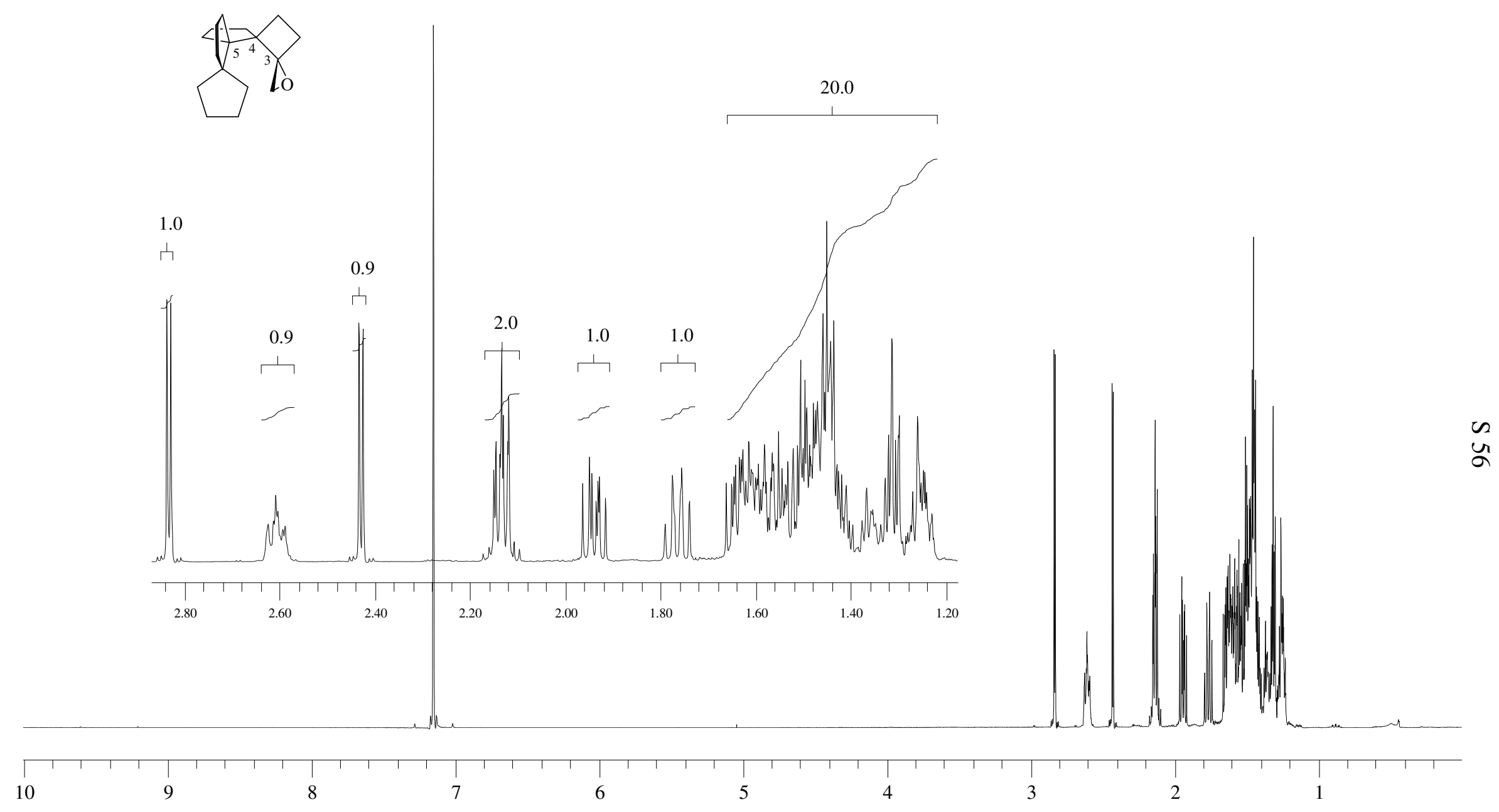

${ }^{1} \mathrm{H}-\mathrm{NMR}$ spectrum $\left(600 \mathrm{MHz}, \mathrm{C}_{6} \mathrm{D}_{6}\right)$ of $\left(3 S^{*}, 4 R^{*}, 5 S^{*}\right)-\mathbf{3 9}$ 


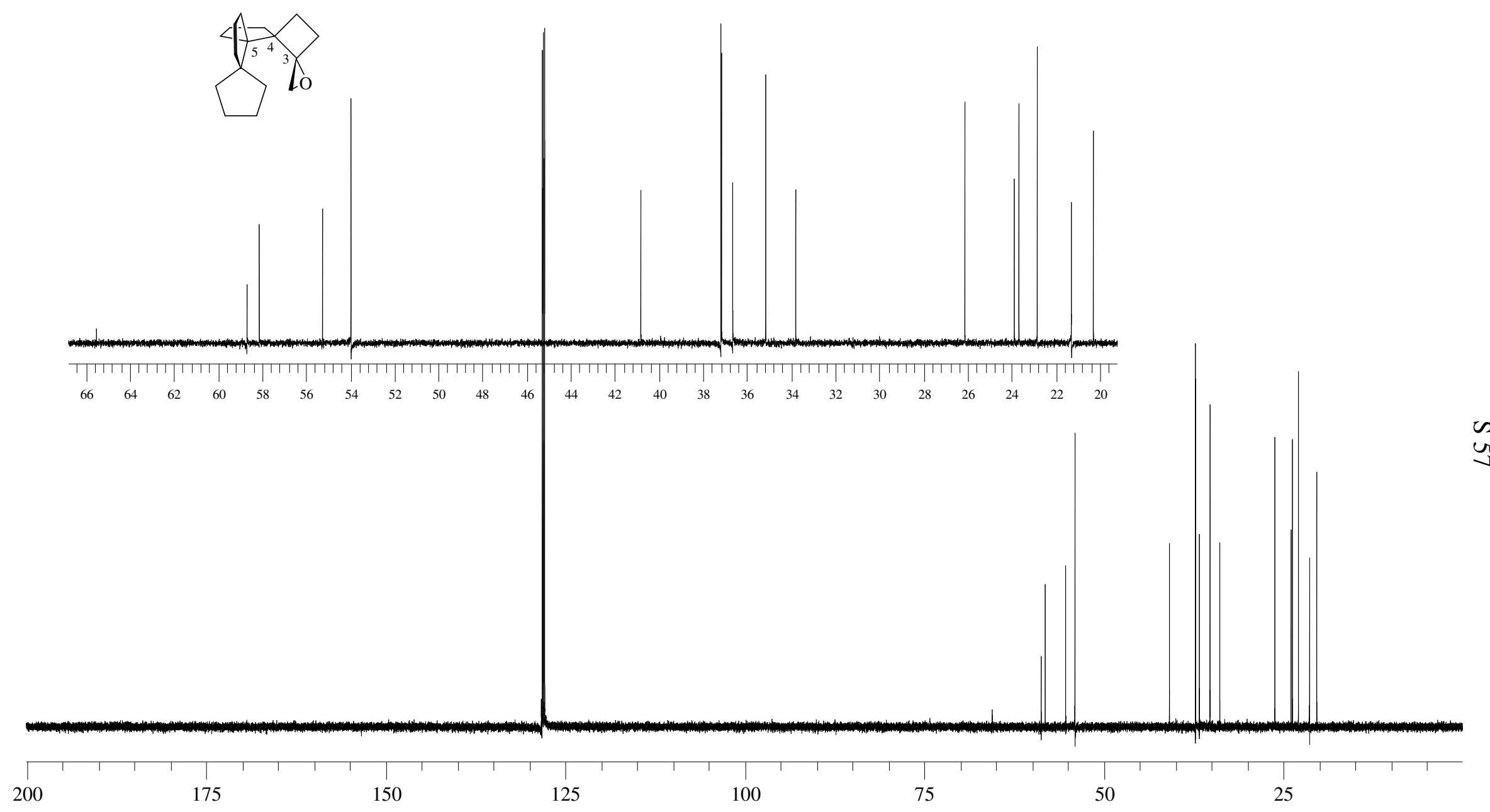

${ }^{13} \mathrm{C}$-NMR spectrum $\left(150.8 \mathrm{MHz}, \mathrm{C}_{6} \mathrm{D}_{6}\right)$ of $\left(3 S^{*}, 4 R^{*}, 5 S^{*}\right)-39$ 


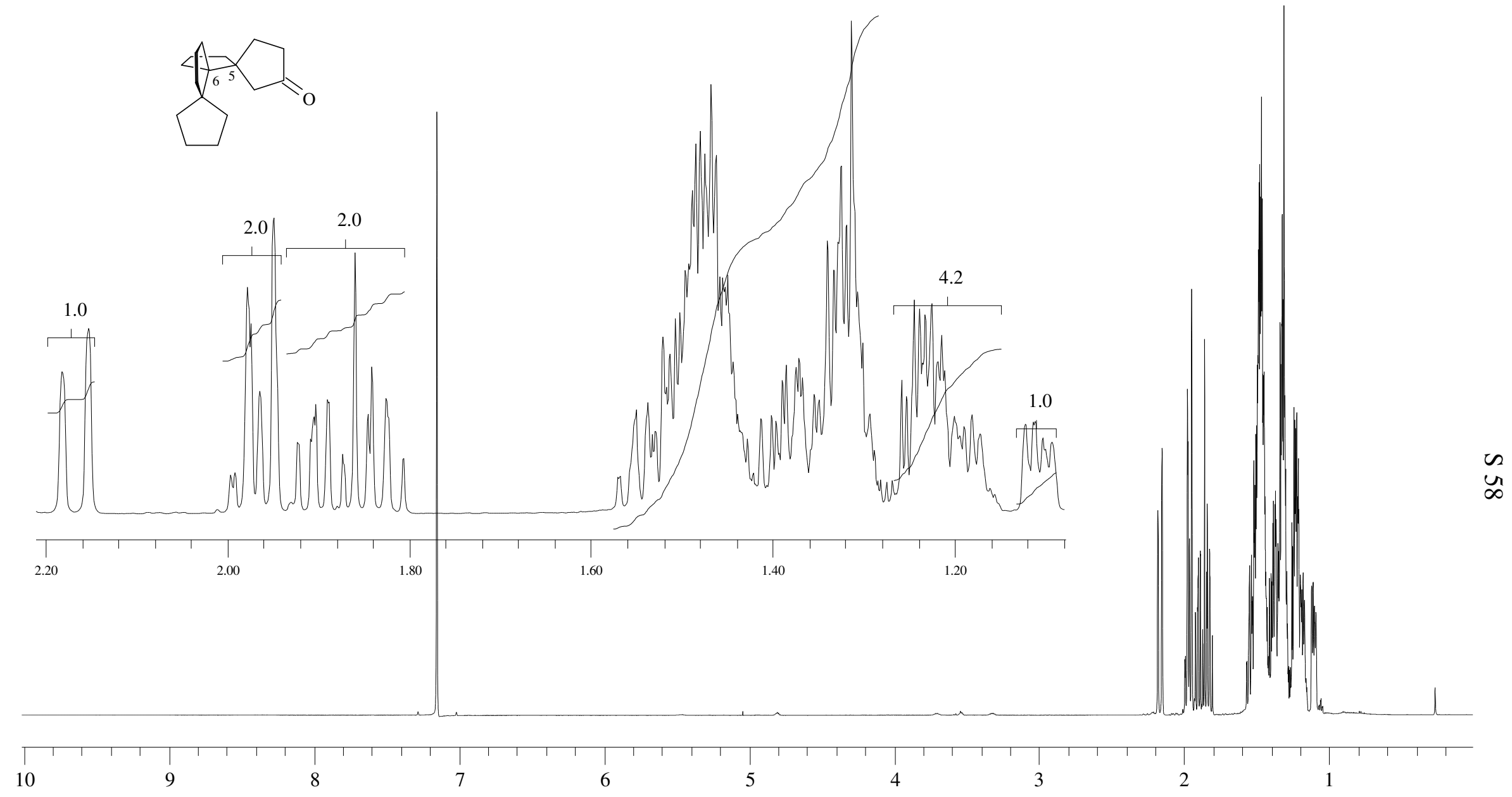

${ }^{1} \mathrm{H}-\mathrm{NMR}$ spectrum $\left(600 \mathrm{MHz}, \mathrm{C}_{6} \mathrm{D}_{6}\right)$ of $\left(5 S^{*}, 6 S^{*}\right)-\mathbf{3 8}$ 


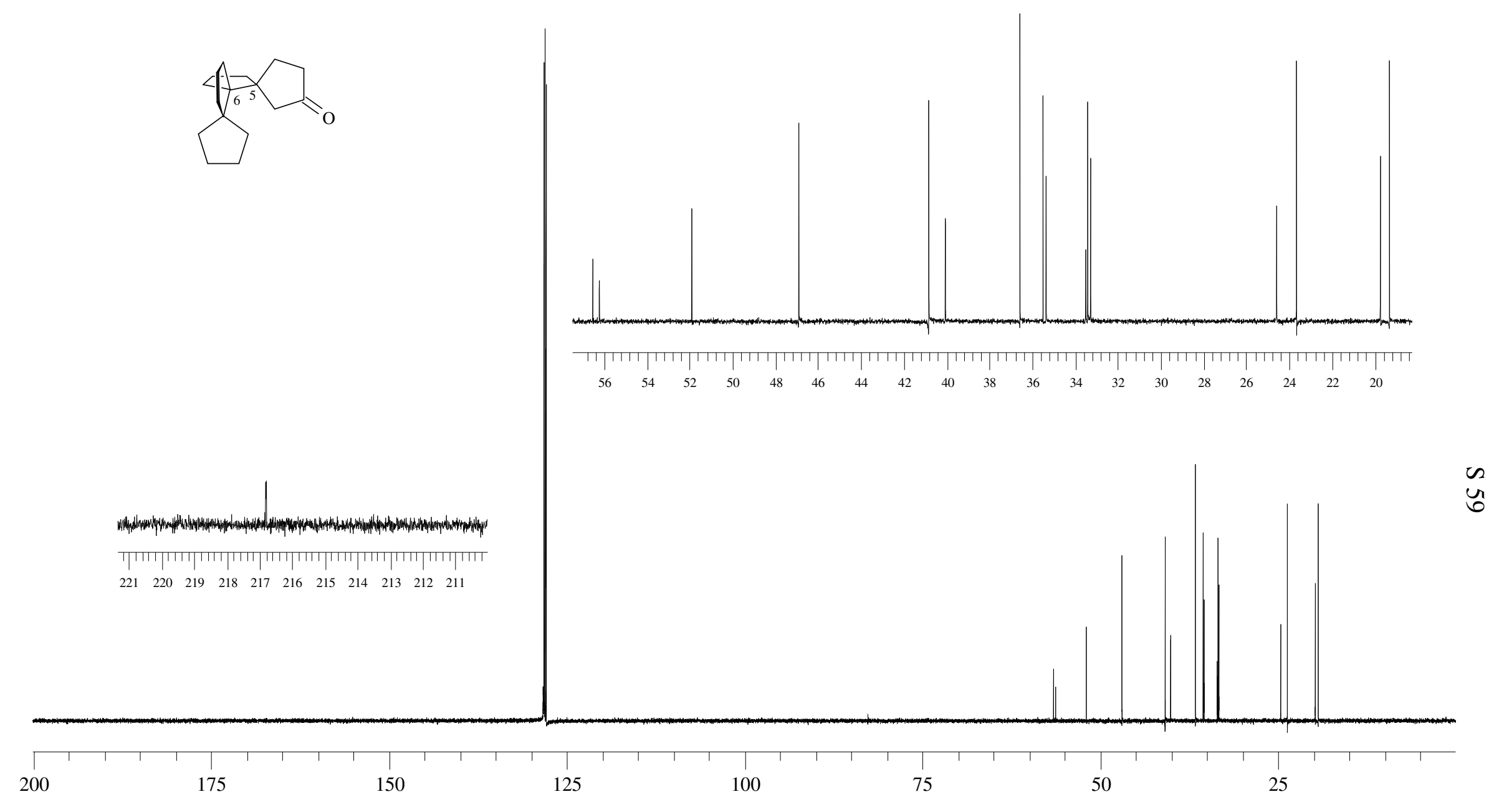

${ }^{13} \mathrm{C}$-NMR spectrum $\left(150.8 \mathrm{MHz}, \mathrm{C}_{6} \mathrm{D}_{6}\right)$ of $\left(5 S^{*}, 6 S^{*}\right)-\mathbf{3 8}$ 